\title{
Observation of excited state charge transfer with fs/ps-CARS
}

by

\begin{abstract}
Alex Jason Blom
A dissertation submitted to the graduate faculty

in partial fulfillment of the requirements for the degree of

DOCTOR OF PHILOSOPHY
\end{abstract}

\author{
Major: Physical Chemistry \\ Program of Study Committee: \\ Hans U. Stauffer, Major Professor \\ Mark S. Gordon, Major Professor \\ Joseph W. Burnett \\ William S. Jenks \\ Victor S.-Y. Lin
}

Iowa State University

Ames, Iowa

2009

Copyright (C) Alex Jason Blom, 2009. All rights reserved. 


\section{TABLE OF CONTENTS}

CHAPTER 1: INTRODUCTION

General Overview

Thesis Organization

References

CHAPTER 2: EXCITED STATE EVOLUTION OF MICHLER'S KETONE IN ITS LOWEST EXCITED SINGLET STATE USING A TIME AND FREQUENCY RESOLVED RAMAN PROBE

Abstract

Introduction

Methods

Results

Discussion

Conclusions

Acknowledgements

References

CHAPTER 3: ANALYSIS OF THE EFFECT OF SOLVATION ON THE VIBRATIONAL STRUCTURE OF COUMARIN 120 USING AN EXPERIMENTAL AND THEORETICAL APPROACH

Abstract

Introduction

Methods

Results and Discussion

Conclusions

Acknowledgements

52

References

CHAPTER 4: EXCITED STATE DYNAMICS OF LOW FREQUENCY MODES OF 4DIMETHYLAMINO-4'NITROSTILBENE IN ACETONITRILE

Abstract

Introduction

Methods

Results

Discussion

72

Conclusion

76

References 
CHAPTER 5: THE BEST OF THE REST

General Overview

Two Photon Absorption of Coumarin 120

Effects of Nanoconfinement on Twisting Dynamics

91

Dye Sensitized Nanoparticles

94

References

97

CHAPTER 6: CONCLUSIONS

Conclusions 


\begin{abstract}
Excited state charge transfer processes are studied using the fs/ps-CARS probe technique. This probe allows for multiplexed detection of Raman active vibrational modes. Systems studied include Michler's Ketone, Coumarin 120, 4-dimethylamino-4'nitrostilbene, and several others. The vibrational spectrum of the para di-substituted benzophenone Michler's Ketone in the first excited singlet state is studied for the first time. It is found that there are several vibrational modes indicative of structural changes of the excited molecule. A combined experimental and theoretical approach is used to study the simplest 7-amino-4-methylcoumarin, Coumarin 120. Vibrations observed in FTIR and spontaneous Raman spectra are assigned using density functional calculations and a continuum solvation model is used to predict how observed modes are affected upon inclusion of a solvent. The low frequency modes of the excited state charge transfer species 4-dimethylamino-4'-nitrostilbene are studied in acetonitrile. Results are compared to previous work on this molecule in the fingerprint region. Finally, several partially completed projects and their implications are discussed. These include the two photon absorption of Coumarin 120, nanoconfinement in cyclodextrin cavities and sensitization of titania nanoparticles.
\end{abstract}




\section{CHAPTER 1: INTRODUCTION}

\subsection{General Overview}

\subsubsection{Charge transfer processes}

The study of ultrafast molecular processes has received great interest in recent decades. ${ }^{1-27}$ These processes, specifically excited state charge transfer, are at the forefront

of current research. ${ }^{10-27}$ At the very basic level these processes involve the subpicosecond evolution of an electronically excited molecule as an electron is transferred from a donor to an acceptor. Electronic excitation induces structural changes within the molecule that depend highly on environmental parameters, especially the solvent environment. These systems are not yet fully understood and efforts continue to unravel how and why such species undergo such dynamical structural changes.

Intramolecular charge transfer systems are composed of a donor group and an acceptor group (see Figure 1.1A). The donor group is generally an electron which can be readily donated. ${ }^{17}$ Acceptor groups are generally electronegative and will readily accept electron density from a donor group. Acceptor groups most often consist of nitro, carbonyl, and cyano groups. ${ }^{17}$ There are varying degrees of charge transfer that can be stabilized in the excited state. Some systems exhibit a partial charge transfer (Figure 1.1B), resulting in a partial positive charge on the donor and a partial negative on the acceptor while others undergo a full transfer of charge in which case there is a full positive charge on the donor and a full negative on the acceptor.

4-dimethylaminobenzonitrile (DMABN) is the prototypical molecule for studying excited state charge transfer processes. This species, shown in Figure 1.2 has been 
studied by numerous groups and several explanations of its observed dynamics have been laid forth. ${ }^{10-17}$ Fluorescent properties of this molecule are highly dependent upon solvent environment. In nonpolar solvents such as hexane DMABN exhibits fluorescence appearing as a mirror image of the absorption spectrum. If the molecule is placed in a slightly polar solvent such as diethyl ether, on the other hand, it exhibits two fluorescence bands, one near that observed in nonpolar solvents and another red-shifted band. ${ }^{17}$ In highly polar solvents the fluorescence dramatically shifts to higher wavelengths and the quantum yield decreases significantly.

Such behavior of DMABN is attributed to an excited state charge transfer. ${ }^{10-17}$ This characteristic is supported by the fluorescence shifting to higher wavelengths in polar solvents, indicating that the dipolar character of the solvent stabilizes a charge separation within the excited species, causing a stabilization of the transient state and a reduction in its energy. Such behavior has been explained in many different ways including a planarization of the amino group, rehybridization of the cyano group, and a 'wagging' of the amino group. The most commonly accepted explanation for this behavior, however, involves a twisting process of the amino group. ${ }^{17}$

Upon excitation, in all solvents, DMABN accesses the Franck-Condon excited state which is structurally very similar to the corresponding ground state. If a nonpolar solvent is present the molecule does not undergo any excited state relaxation processes and fluoresces directly back to the ground state. Polar solvents are able to stabilize the charge separation by rearranging themselves around the excited species. In slightly polar solvents a partial charge transfer is stabilized and fluorescence is observed from the molecules that undergo this partial charge transfer (red-shifted band) and from those that 
do not (normal band). In highly polar solvents a complete charge transfer occurs. The decrease in fluorescence intensity in highly polar solvents is associated with a twist of the donor group with respect to the acceptor. This twist results in the formation of a metastable excited state species termed the twisted intramolecular charge transfer (TICT) state for obvious reasons.

With the extensive interest in DMABN has come a desire to study and understand molecules similar to it. ${ }^{17-27}$ Figure 1.3 illustrates several species that have been scrutinized in order to understand charge transfer processes associated with their observed excited state dynamics. All of the structures in Figure 1.3 contain an amino group as the donor. This is the most commonly encountered donor group as it readily can be ionized to form a charge transfer state and can twist to facilitate this transfer.

CT molecules have traditionally been studied using electronic spectroscopy. The advent of the laser in the mid $-19^{\text {th }}$ century led to the development of numerous spectroscopic methods for studying the excited-state evolution of such systems. ${ }^{1-7}$ Subsequent advances including pulsed lasers with femtosecond duration have allowed enhancement of the time resolution of these methods and brought with them a distinct ability to monitor molecular dynamics with great sensitivity. Current laser systems allow researchers to measure the evolution of an excited molecule's electronic spectrum with sub-100 fs time resolution.

\subsubsection{Methods for studying charge transfer}

For transient studies ultrashort laser pulses are used. The temporal and spectral characteristics of these pulses are related through Fourier transforms and their product is 
governed by the Heisenberg uncertainty principle. ${ }^{1-7}$ A modified version of the principle restricts the time and frequency resolution that can be achieved simultaneously. The product $\mathrm{dt} \cdot \mathrm{dv}$, the temporal duration and spectral width, respectively, cannot exceed a certain value. This value depends upon the type of pulse being used. ${ }^{1}$ The shortest pulse duration leads to the broadest spectrum and is termed the transform limit. A good example is a $120 \mathrm{fs}$ pulse which has a $120 \mathrm{~cm}^{-1}$ bandwidth. Longer duration pulses are narrower while shorter ones have more bandwidth. These characteristics of ultrashort pulses must be considered when determining the type of method used for studying transient species.

\subsubsection{Electronic methods}

Time resolved electronic spectroscopy allows researchers to monitor excited state dynamics with very high time resolution. With current laser sources having sub-100 fs duration, electronic processes occurring on these timescales can be monitored. There are two main techniques used to monitor the time resolved electronic spectrum of an excited species: fluorescence upconversion and transient absorption. Fluorescence upconversion has the distinct ability to measure the emission spectrum of a molecule as it is evolving along its excited state potential energy surface. ${ }^{28}$ This technique operates by preparing a transient species through an energetic excitation pulse. Fluorescence from the sample is separated from the incident beam and molecular scatter such as Raman scatter are removed using filters. The signal is then focused into a mixing crystal, where it is combined or 'upconverted' with a gate beam of known character. Combination with this gate beam increases the intensity of the fluorescence to ease data acquisition. ${ }^{28}$ The 
upconverted signal is then collected though the use of a detector which is generally a photon counter, although other detection schemes are available. During data analysis the signal can be 'downconverted' to determine its original wavelength.

The second time resolved electronic technique is known by several names but will be called transient absorption/emission here. ${ }^{29}$ This technique is capable of monitoring simultaneously the absorption and emission spectrum of a transient species. Again, an excitation pulse prepares an excited molecule. A second beam, termed a probe beam, is also incident upon the sample and stimulates electronic transitions from the excited species. Once excited, a molecule may undergo an excited state 'transient absorption' to a higher-lying excited state via absorption of the probe pulse. The transient species may also experience an excited state 'transient emission' to the ground state. Finally, the molecule may remain static and not interact with the probe pulse. Choice of probe beam depends on the experimental setup but often a white light continuum is used so that a wide range of probe wavelengths are incident upon the sample simultaneously and a large region of the electronic spectrum can be measured in a single experiment. The probe beam is spatially separated from the excitation pulse and spectrally dispersed so that changes in its wavelength components can be monitored.

Both fluorescence upconversion and transient absorption/emission are useful, especially if used together. The main drawback of the latter technique is the possibility of a subset of molecules undergoing excited state absorption while another set undergoes excited state emission. If this occurs it is nearly impossible to separate components from the two processes. In most cases one process will outweigh the other. This is where fluorescence upconversion can be very helpful. If both techniques are used on a single 
system they can be compared so the absorptive and emissive components can be better identified and understood.

These two techniques are extremely useful for measuring electronic spectra of transient systems and are commonplace in current research. The amount of information gained from using such electronic methods can be staggering. Timescales associated with solvation, charge transfer, and a variety of other processes can be found using these two methods. ${ }^{28,29}$ Electronic methods are especially useful for determining if charge transfer is present. As mentioned for DMABN, the presence of a red-shift of the fluorescence spectrum in polar solvents is an indicator of charge transfer.

There are certain aspects of a dynamical process that cannot be discerned using electronic methods, however. ${ }^{1-4}$ Ultrafast laser pulses are inherently broadband, making it difficult to evaluate structural changes occurring except when they are very elementary. The case of charge transfer may be a 'simple' example, especially since there is a high degree of solvatochomism associated with such an occurrence. This lack of structural information is a huge negative for electronic spectroscopy. These methods provide a great starting point for vibrational spectroscopy, however...

\subsubsection{Vibrational methods}

The broadband nature of ultrafast pulses severely limits their ability to determine structural changes associated with excited molecules. Vibrational methods, specifically transient infrared (IR) and transient Raman methods, however, allow for direct observation of structural changes in such species. ${ }^{1-7}$ Linewidths associated with vibrations are considerably narrower than electronic transitions and can have dephasing 
times on the order of picoseconds. IR and Raman methods, of course, are complementary with the selection rules of each governing the types of vibrations that are active in each technique. These methods are able, much like their electronic counterparts, to monitor ultrafast molecular processes with sub-100 fs resolution.

Most approaches to excited state vibrational spectroscopy take the form of a pump-probe technique. ${ }^{1-7}$ The pump pulse is very energetic and induces an excitation within the molecule while the probe pulse(s) monitor(s) how the molecule is affected by this perturbation. Of course if the probe setup is used without the pump it is possible to observe the ground state spectrum of a molecule. Both transient methods are useful for determining structural changes in molecules.

Transient IR spectroscopy typically uses an ultraviolet or IR pump pulse followed by an IR probe pulse. ${ }^{1-5}$ Typically these pulses are sub-picosecond in duration and are capable of at least $150 \mathrm{~cm}^{-1}$ bandwidth. The bandwidth can change based on the duration of the pulse used and the Heisenberg uncertainty principle. Transient IR methods to this point have largely been used to study metal-carbonyl interactions and polypeptides in the ground state. By monitoring the change of frequency associated with a particular vibrational mode of interest, such as a hydrogen bond, the interactions in a molecule can be found and its structure elucidated. The frequencies available for transient IR are somewhat limited and must be tuned to look at each portion of the vibrational spectrum. ${ }^{4}$ In addition, care must be taken to ensure pulses are not distorted when traveling through samples.

Transient Raman spectroscopy is very useful for studying structural changes within molecules. ${ }^{1-7,30}$ It is common to use four wave mixing processes in transient 
Raman experiments in which three input beams induce a scattering process in the sample which results in a fourth (signal) beam being outputted. Specifically, coherent anti-Stokes Raman scattering (CARS) (Figure 1.4) has allowed researchers to monitor molecular dynamics for decades. ${ }^{3,5}$ Selection of CARS over spontaneous Raman spectroscopy is due to the spectral coverage, discrimination against fluorescence and stimulated nature of the technique. Under the right conditions CARS can yield greatly increased signal compared to spontaneous Raman and yields the entire fingerprint region of the spectrum without having to tune any pulses. ${ }^{30}$ A brief description of this method follows.

The polarization of a molecule may be expanded in a Taylor series of electric field interactions which induces an $n+1$ scattering process in the sample. ${ }^{3}$

$$
\begin{aligned}
& \mathbf{P}=\mathbf{P}^{(1)}+\mathbf{P}^{(2)}+\mathbf{P}^{(3)}+\ldots \\
& \mathbf{P}=\chi^{(1)} \mathbf{E}+\chi^{(2)} \mathbf{E E}+\chi^{(3)} \mathbf{E E E}
\end{aligned}
$$

In these equations bold letters are vectors and the electric fields should be considered as tensor products with the $\chi$ terms. Each $\chi$ term represents a molecular property that is different for every sample and contains information about the transition dipoles within a system.

Conventional Raman scattering is governed by the first term in equation 1.2 , the first order polarization of a system. Signals associated with this first order process are low unless the excitation pulse is tuned to an electronic resonance, resulting in a dramatic increase in signal. Four wave mixing spectroscopies are governed by the third term in equations 1.1 and 1.2. The third order polarization involves a molecule's interaction with three electric fields of the form in equation 1.3. 


$$
\mathbf{E}(\omega, z, t)=\frac{1}{2}\left[\mathbf{E}(\omega, z, t) e^{-i(\omega t-k z)}+c . c .\right]
$$

Interaction with three electromagnetic fields to output a fourth field results in several widely known experimental techniques, including coherent anti-Stokes Raman scattering (Figure 1.5). ${ }^{1-7,30}$ The tensorial nature of the interaction of $\chi^{3}$ with the electric fields means that there are a number of different interactions possible with three incident beams. The scattering process is governed by wave-vector phase matching conditions which must fulfill the following equations.

$\mathbf{P}_{i}^{(3)}\left(\omega_{4}\right)=\mathbf{C} \chi_{i j k l}^{(3)}\left(-\omega_{4}, \omega_{1},-\omega_{2}, \omega_{3}\right) \mathbf{E}_{j}\left(\omega_{1}, z\right) \mathbf{E}_{k}^{*}\left(\omega_{2}, z\right) \mathbf{E}_{l}\left(\omega_{3}, z\right) e^{-i\left[\omega_{4} t-\left(\mathbf{k}_{1}-\mathbf{k}_{2}+\mathbf{k}_{3}\right) z\right]}+$ c.c.

$$
\begin{aligned}
& \omega_{4}=\omega_{1}-\omega_{2}+\omega_{3} \\
& \mathbf{k}_{4}=\mathbf{k}_{1}-\mathbf{k}_{2}+\mathbf{k}_{3}
\end{aligned}
$$

In equation 1.5 and $1.6, \omega_{4}$ and $\mathbf{k}_{\mathbf{4}}$ are the frequency and direction of the scattered radiation for the CARS process, respectively. In the CARS process $\omega_{1}$ interacts with the sample to populate a virtual state. This virtual state dephases on very fast timescales and is only present while the electric field is populating it. ${ }^{30}$ The second pulse, $\omega_{2}$ then depopulates the virtual state to populate vibrationally excited states. These first two interactions create a coherent superposition of vibrationally excited states. The third pulse, $\omega_{3}$ then populates a second virtual state. Population of this final virtual state initiates the scattering process.

Current experimental considerations allow the uncertainty principle to be circumvented in certain circumstances. ${ }^{4,30}$ Several probe methods such as femtosecond stimulated Raman scattering and fs/ps-CARS use a combination of broadband and 
narrowband pulses to achieve multiplex detection of Raman active vibrations. ${ }^{4,30}$ These methods allow for temporal resolution greater than $100 \mathrm{fs}$ and spectral resolution of better than $10 \mathrm{~cm}^{-1}$. The availability of multiplexed techniques eases the study of excited states of molecules.

Using a pump-probe setup the fs/ps-CARS probe follows an excitation 'pump' pulse to monitor the vibrational dynamics induced by such a perturbation. This allows for all but the fastest structural changes to be monitored. In this way, if a specific molecular process such as charge transfer process is suspected to occur, specific vibrations associated with the donor and acceptor can be monitored in real time. Comparison of transient spectra with ground state spectra can then help determine exactly how the molecule is changing.

\subsubsection{Computational Techniques}

Assignment of Raman active vibrations is not always straightforward. Density Functional Theory calculations are often used in addition to vibrational techniques to assign ground state vibrations. ${ }^{28,31,32}$ Understanding the ground state configuration of a molecule is the first step to understanding how it changes following a perturbation such as electronic excitation. Several of the studies discussed herein include computational calculations in order to better understand an experimentally observed system.

\subsection{Thesis Organization}

This thesis describes the use of the fs/ps-CARS probe technique for monitoring dynamics of charge transfer in excited state molecules. The second chapter discusses 
experiments aimed at elucidating the structural changes of Michler's Ketone in its first excited singlet state and the structural changes resulting from charge transfer processes. The third chapter is concerned with the vibrational structure of coumarin 120 as studied with experimental and computational techniques. Specifically, the assignment of experimentally observed vibrations is emphasized. The fourth chapter regards the use of the fs/ps-CARS technique to study low frequency vibrational modes in the ground and excited state of 4-dimethylamino-4'-nitrostilbene. Finally, chapter five outlines several projects that are ongoing and may require future work.

\subsection{References}

1. H. Hamaguchi and T. L. Gustafson, Annu Rev. Phys. Chem., 45, 593 (1994)

2. E. T. J. Nibbering, H. Fidder and E. Pines, Annu. Rev. Phys. Chem., 56, 337 (2005)

3. W. M. Tolles, J. W. Nibler, J. R. McDonald and A. B. Harvey, Appl. Spectrosc., 31, 253 (1977)

4. P. Kukura, D. W. McCamant and R. A. Mathies, Annu. Rev. Phys. Chem., 58, 461 (2007)

5. M. D. Fayer, Ultrafast Infrared and Raman Spectroscopy, Dekker, 2001

6. J.-C. Diels and W. Rudolph, Ultrashort Laser Pulse Phenomena, Academic Press, 1996

7. S. De Silvestri, G. Cerullo, and G. Lanzani, Coherent Vibrational Dynamics, CRC, 2008

8. A. Demeter and K. A. Zachariasse, Chem. Phys. Lett., 308, 699 (2003)

9. J. Dreyer and A. Kummrow, J. Am. Chem. Soc., 122, 2577 (2000)

10. S. I. Druzhinin, N. P. Ernsting, S. A. Kovalenko, L. W. Lustres, T. A. Senyushkina, and K. A. Zachariasse, J. Phys. Chem. A, 110, 2955 (2000) 
11. W. M. Kwok, C. Ma, D. Phillips, P. Matousek, A. W. Parker and M. Towrie, J. Phys. Chem. A, 104, 4188, (2000).

12. H. Okamoto, J. Phys. Chem. A, 104, 4182 (2000)

13. H. Okamoto, H. Inishi, Y. Nakamura, S. Kohtani, and R. Nakagaki, J. Phys. Chem. A, 105, 4182 (2001)

14. K. A. Zachariasse, M. Grobys and E. Tauer, Chem. Phys. Lett., 274, 372 (1997)

15. K. A. Zachariasse, Chem. Phys. Lett., 320, 8 (2000)

16. K. Rotkiewicz, K. H. Grellman and Z. R. Grabowski, Chem. Phys. Lett., 19, 315 (1973)

17. Z. R. Grabowski, K. Rotkiewicz and W. Rettig, Chem. Rev., 103, 3899 (2003)

18. H. Görner and H. J. Kuhn, Adv. Photochem., 19, 1 (1995)

19. Y. V. Il'chev, W. Kühnle and K. A. Zachariasse, Chem. Phys., 211, 441 (1996)

20. W. Rettig and W. Majenz, Chem. Phys. Lett., 154, 335 (1989)

21. R. Lapouyade, A. Kuhn, J. F. Letard and W. Rettig, Chem. Phys. Lett, 208, 48 (1993)

22. J. Oberlé, E. Abraham, G. Jonusauskas and C. Rulliére, J. Ramam. Spectrosc, 31, $311(2000)$

23. J. Oberlé, G. Jonusauskas, E. Abraham, R. Lapouyade, and C. Rulliére, B. Chem. Soc. Jpn., 75, 1041 (2002)

24. H. Gruen and H. Görner, J. Phys. Chem., 93, 7144 (1989)

25. V. M. Farztdinov and N. P. Ernsting, Chem. Phys, 277, 257 (2002)

26. T. Vijayakumar, I. Hubert Joe, C. P. Reghunadhan Nair, V. S. Jayakumar, Chem. Phys., 343, 83 (2008).

27. B. M. Prince, B. D. Prince, A. Chakraborty, M. N. Slipchenko, and H. U. Stauffer, J. Raman. Spectrosc., manuscript in preparation

28. R. Schanz, S. A. Kovalenko, V. Kharlanov and N. P. Ernsting, Appl. Phys. Lett., 78, $566(2001)$ 
29. V. I. Klimov and D. W. McBranch, Opt. Lett., 23, 277 (1998)

30. B. D. Prince, A. Chakraborty, B. M. Prince, M. S. Gordon, and H. U. Stauffer, submitted to J. Phys. Chem. A

31. M. W. Schmidt, K. K. Baldridge, J. A. Boatz, S. T. Elbert, M. S. Gordon, J. H. Jensen, S. Koseki, N. Matsunaga, K. A. Nguyen, S. J. Su, T. L Windus, M. Dupuis, J. A. Montgomery, J. Comp Chem 14, 1347 (1993)

32. C. J. Cramer, Essentials of Computational Chemistry: Theories and Models; Wiley, 2002. 

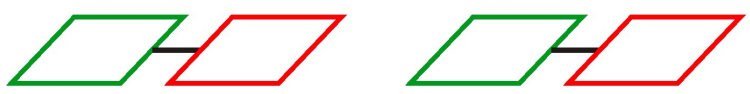
A)

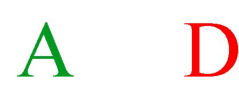
B)

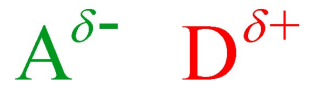

Figure 1.1 - A) Donor-acceptor species pre-charge transfer B) Donor-acceptor species after partial charge transfer.

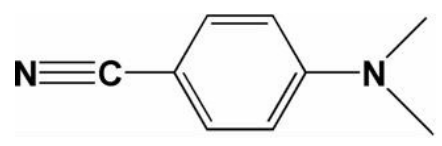

Figure 1.2 - Structure of 4-dimethylamino-benzonitrile.<smiles>CN(C)c1ccc([N+](=O)[O-])cc1</smiles><smiles>[NH]c1ccc([N+](=O)[O-])cc1</smiles><smiles>CN(C)c1ccc(CCc2ccc([N+](=O)[O-])cc2)cc1</smiles><smiles>CN(C)c1ccc(CCc2ccc(C#N)cc2)cc1</smiles><smiles>CN(C)c1ccc(C(=O)c2ccc(N(C)C)cc2)cc1</smiles>

Figure 1.3 - Representative charge transfer species. 


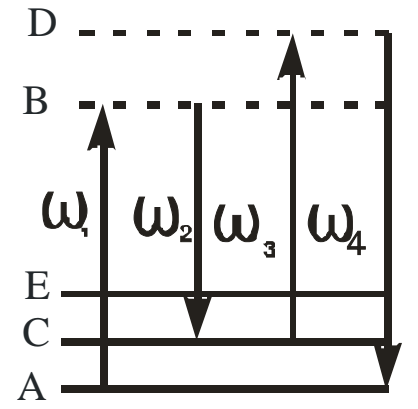

Figure 1.4 - Graphical representation of the CARS process. 


\title{
CHAPTER 2: EXCITED STATE EVOLUTION OF MICHLER'S KETONE IN ITS LOWEST EXCITED SINGLET STATE USING A TIME AND FREQUENCY RESOLVED RAMAN PROBE
}

\author{
Alex J. Blom, Benjamin D. Prince, Mikhail N. Slipchenko, and Hans U. Stauffer
}

\subsection{Abstract}

Vibrational dynamics of Michler's Ketone in its first excited singlet state are monitored using the fs/ps-CARS probe. Results in deuterated methanol show a very fast $(<150 \mathrm{fs})$ increase in the frequency of the phenyl-N stretch with a concomitant increase in the frequency of the phenyl-N + phenyl CC mode, indicating the production of an intramolecular charge transfer state. Results in deuterated dimethyl sulfoxide indicate the production of a long-lived state, likely a twisted intramolecular charge transfer state through dynamics of the phenyl-N + phenyl C-C stretch. Additionally, low frequency studies in dimethyl sulfoxide indicate a torsional mode near $280 \mathrm{~cm}^{-1}$ with dynamics similar to the phenyl-N + phenyl C-C mode.

\subsection{Introduction}

Twisted intramolecular charge transfer (TICT) species have been under intense investigation in recent years because of their excited state (ES) dynamics. References 1-5 and citations within give a good background of work in this field. Such substances contain an electron accepting (A) group and an electron donating (D) group (Figure 2.1). ${ }^{1}$ The A group is generally a cyano, carbonyl, or nitro group and the D group is often an 
amino group of differing alkyl substituents. So-called donor-acceptor (D-A) species are polar and the magnitude of their dipole increases as solvent polarity increases and charge transfer from D to A is stabilized.

Excited state behavior of D-A molecules varies widely according to solvent (Figure 2.1). ${ }^{1}$ In nonpolar solvents there are generally no ES dynamics to speak of with the molecule fluorescing directly back to the ground state from the Franck Condon (FC) state. This behavior is illustrated in Figure 2.1 (purple). In slightly dipolar solvents such as ethyl ether the solvent is capable of stabilizing a charge separation of the D-A species in its ES, resulting in a portion of the ES species becoming lower in energy. In this case the system will display fluorescence from the FC state as well as red-shifted fluorescence from the stabilized molecules. This is indicated by the green fluorescence in Figure 2.1.

In the extreme case of a highly polar solvent such as dimethyl sulfoxide (DMSO) or a polar protic solvent such as methanol $(\mathrm{MeOH})$ the charge separation between the $\mathrm{D}$ and A groups can go to such an extent that a full electron is transferred between the two. This charge transfer results in a positive charge on the D group and a negative charge on the A group (see Figure 2.1). Here the D group, in order to fully transfer the charge tends to undergo twisting, effectively decoupling it from the rest of the system. This is the TICT state. Once in this state the molecule shows very little fluorescence due to a lack of overlap between the twisted excited state with the ground state. This state returns to the ground state via a nonradiative process, generally internal conversion.

4,4'-bis(N,N-dimethylamino)-benzophenone, commonly referred to as Michler's Ketone (MK), whose structure is shown in Figure 2.2, is thought to undergo a TICT process in polar solvents. Recent experimental and theoretical papers have suggested a 
complicated evolution of the excited singlet state of MK. ${ }^{2,3,6,7}$ This evolution is highly solvent-dependent with several conformations being present in highly polar solvents. ${ }^{2}$ Glasbeek et al used fluorescence upconversion to monitor the time-resolved fluorescence of MK and its blocked counterpart (BMK). ${ }^{6}$ In BMK the phenyl rings are fused together so they cannot twist. This group observed a Stokes shift of the emission of MK in polar solvents, consistent with previous steady-state experiments, and was accompanied by an intensity decay. ${ }^{3,9}$ The blocked compound underwent a Stokes shift but did not display the decay in emission intensity. Their interpretation was that a combination of solvation and phenyl ring twisting caused the observed shift and decay of the fluorescence.

Palit and coworkers used transient absorption methods to monitor the excited state electronic spectrum of MK in a plethora of solvents. ${ }^{2}$ In ethyl acetate they concluded that the molecule undergoes a planarization out of the initially twisted configuration, followed by fluorescence from a partially stabilized excited state. In this solvent they did not find evidence for a TICT state. In highly polar solvents like ACN and DMSO, as well as in polar protic solvents like $\mathrm{MeOH}$, the molecule displayed dynamics consistent with formation of a TICT state which they observed through multiple intermediate states in time-resolved electronic spectra. They also determined timescales for the formation and decay of each species formed in the excited state. Their overall picture was that following excitation the molecule undergoes a planarization process and a partial charge transfer to form the intramolecular charge transfer (ICT) state. The ICT state, in highly polar solvents, then undergoes twisting of a single dimethylamino group to $90^{\circ}$ relative to the plane of the phenyl rings, completing the charge transfer to the carbonyl group and resulting in the TICT state. 
Computational studies have also indicated the presence of a TICT state in this molecule. ${ }^{7,8}$ In the ground state MK is not planar, but twisted. ${ }^{2,7,8}$ The angle reported varies from 40 to 60 degrees but all groups have reported that the molecule is twisted in its ground state. Granchak used force field (PM3) and ab initio (Hartree-Fock) methods to calculate dependence of the molecule's energy in the ground and excited states on phenyl twist angle. ${ }^{7}$ They also conducted calculations in the ground and excited state where the twist angle of the amino groups was changed relative to the phenyl rings. Their results suggested that a planar MK with one dimethylamino group twisted to 90 degrees was the lowest energy excited state configuration of this molecule in ethanol.

Pal and coworkers used time dependent density functional theory to study the excited state evolution of MK in several solvents. ${ }^{8}$ Their results largely agreed with the general picture laid out by Palit and company. ${ }^{2}$ These calculations used continuum solvation models to determine the evolution of MK when subjected to varying polarity solvents.

There have been very few vibrational studies of MK thus far. Two papers published by Hamaguchi studied the triplet state of MK. ${ }^{10,11}$ In polar solvents, however, the intersystem crossing efficiency is low and the molecule is likely to remain in the singlet state. The $S_{1}$ state, to our knowledge, has not been studied. Determining the vibrational evolution of MK in its first excited singlet state can help further understand this very interesting system.

In this paper a simultaneously time- and frequency-resolved vibrational probe termed fs/ps-CARS is used to monitor the sub-picosecond vibrational dynamics of MK in deuterated DMSO and deuterated $\mathrm{MeOH}$. The fs/ps-CARS probe technique allows for 
multiplexed detection of Raman active vibrations. ${ }^{12}$ This technique has been shown to be very sensitive to excited state vibrations in molecules and is a valid tool for studying this system. ${ }^{13}$ This probe is also used to study the low-frequency modes of MK in DMSO. Peak assignments of the ground state are made with the assistance of computational methods.

\subsection{Methods}

\subsubsection{Experimental Setup}

Details of the fs/ps-CARS setup have been described previously. ${ }^{12}$ Briefly, pulses at $800 \mathrm{~nm}(\sim 50 \mathrm{fs})$ are generated using a Ti:Sapphire laser system operating at $1 \mathrm{kHz}$ and $2 \mathrm{~mJ} /$ pulse. The fundamental beam is divided into three parts, a portion of which is used to prepare broadband ultrafast pulses via use of an optical parametric amplifier (OPA). After combination with the $800 \mathrm{~nm}$ beam, the OPA generates two pulses $\left(\omega_{1}=19570\right.$ $\mathrm{cm}^{-1}[511 \mathrm{~nm}]$; bandwidth $\Delta \omega=300 \mathrm{~cm}^{-1}$ and $\omega_{2}=18210 \mathrm{~cm}^{-1}[549 \mathrm{~nm}] ; \Delta \omega=350$ $\mathrm{cm}^{-1}$ ). For low frequency studies these frequencies are tuned so the two beams are nearly degenerate $\left(\omega_{1}=19084 \mathrm{~cm}^{-1}[524 \mathrm{~nm}]\right.$ and $\left.\omega_{2}=18726 \mathrm{~cm}^{-1}[534 \mathrm{~nm}]\right)$.

A second portion of the $800 \mathrm{~nm}$ pulse is used to produce an ultrafast $400 \mathrm{~nm}$ pulse through use of a frequency doubling crystal. This $400 \mathrm{~nm}$ pulse is used to access excited states in molecular species and is denoted $\omega_{\mathrm{ex}}$. The final portion of the $800 \mathrm{~nm}$ beam us used to generate a narrowband $\left(\sim 8 \mathrm{~cm}^{-1}\right)$ picosecond pulse using a grating stretchercompressor apparatus. ${ }^{14}$ This stretcher-compressor has an adjustable slit that allows selection of the desired pulse bandwidth. 
Beams used in fs/ps-CARS experiments are directed into the sample in a folded BOXCARS geometry. The signal beam is spatially separated from the input beams and directed into a spectrometer where it is spectrally dispersed. The spectrometer itself is made up of a monochromator (Jarrell Ash, $f=300 \mathrm{~mm}$ ) which is outfitted with a grating (1200 line/mm) and a Princeton Instruments 1100 element array CCD detector. This detection scheme allows resolution of $\sim 1.2 \mathrm{~cm}^{-1} /$ pixel in the frequency range being used. In order to calibrate the spectrometer a known standard was employed consisting of a 50:50 acetonitrile:toluene mixture. A Raman spectrum of this standard was then compared to tabulated frequencies in the $1100-1800 \mathrm{~cm}^{-1}$ range for fingerprint region studies. ${ }^{15}$ For low frequency studies dimethyl sulfoxide was used as an internal standard and the calibration was performed on the three solvents modes near $300 \mathrm{~cm}^{-1}$. Measured frequencies are reproducible within $2 \mathrm{~cm}^{-1}$. To prepare an excited state system, $\omega_{e x}$ is directed along the central axis of the folded BOXCAR with a PC-controlled delay stage used to adjust its delay relative to the fs/ps-CARS probe. Polarizations for all beams is parallel.

Two color transient studies of the excited electronic state were conducted using $\omega_{\text {ex }}$ to prepare an electronically excited state followed by either $\omega_{1}$ or $\omega_{2}$ as probe to study the dynamics within the system. The timing of the excitation pulse is controlled with a computer-controlled delay stage. A chopper operating at a frequency of $500 \mathrm{~Hz}$ was used to remove contributions from the probe beam. Following interaction at the sample the probe beam is spatially separated from the $\omega_{\mathrm{ex}}$, collected using a photodiode and its intensity monitored using a lock-in amplifier. 


\subsubsection{Materials}

All materials were used without further purification. Michler's Ketone (4,4'-Bisdimethylaminobenzophenone) was obtained from Acros (98\%). Deuterated methanol $\left(d_{4}\right)$ and deuterated dimethyl sulfoxide $\left(d_{6}\right)$ were obtained from Cambridge Isotopes. Reagent grade dimethyl sulfoxide (DMSO) was obtained from Fisher. MK solutions were prepared by dissolving the solid MK in a solvent with typical concentrations in $d_{4}-\mathrm{MeOH}$ ranging from 5-10 $\mathrm{mM}$ and those in $d_{6}$-DMSO or regular DMSO ranging from 40-60 $\mathrm{mM}$. The sample was placed in the gap between two 2" diameter fused silica windows $(220 \mu \mathrm{m})$ and rotated at a rate of $\sim 30 \mathrm{~Hz}$ to allow the sample to be fully refreshed from pulse-to-pulse $(1 \mathrm{kHz})$.

\subsubsection{Computational Methods}

Geometry optimization and vibrational analysis of MK have been carried out at the Density Functional Theory (DFT) level using the B3LYP functional. All calculations were conducted using GAMESS. ${ }^{16}$ This functional has been shown by several groups to

give reliable geometries and vibrational frequencies in conjugated organic systems. ${ }^{17-19}$ The optimized geometry of MK was found using the $6-31+\mathrm{G}(\mathrm{d}, \mathrm{p})$ basis set at the specified level of theory. Hessian calculations were then conducted on the final geometry to determine if the molecule was at a stationary point. These Hessian runs also calculated harmonic vibrational frequencies. Finally, Raman and infrared intensities were found using the GAMESS default field strengths. ${ }^{16}$ Since this method tends to overestimate vibrational frequencies, the calculated frequencies were scaled by a standard tabulated factor of $0.964 .^{20}$ 


\subsection{Results}

\subsubsection{Two Color Pump-Probe Results}

Steady-state absorption and emission spectra for MK in several solvents have been published elsewhere and will not be reproduced here. ${ }^{2,3,6}$ Experimental considerations, specifically the availability of a $400 \mathrm{~nm}$ excitation pulse resulted in highly polar solvents being used in these studies. Previous work has shown that in nonpolar solvents the absorption spectrum of MK trails off near $400 \mathrm{~nm}$ and only highly polar aprotic solvents such as DMSO or polar protic solvents such as methanol cause a red shift

of the absorption spectrum sufficient to excite to $S_{l}$ with this wavelength. ${ }^{2,3,6}$

One advantage of exciting MK at the red edge of its absorption spectrum in DMSO or $\mathrm{MeOH}$ is that it can be assumed that there is no contribution from vibrational cooling since excitation is directly to the lowest vibrational states in $S_{l}$. This indicates that all dynamics are directly related to structural changes. In addition, it should be noted that the hydrogenated solvents DMSO and $\mathrm{MeOH}$ were replaced with their deuterated counterparts for experiments in the fingerprint region. After initial experiments were conducted it was determined that the methyl vibrations in these solvents overlapped with excited state vibrations of MK. These same vibrations in $d_{6}$-DMSO and $d_{4}-\mathrm{MeOH}$ were downshifted out of the region of interest. Low frequency studies were conducted with hydrogenated DMSO because the low frequency modes of $d_{6}$-DMSO interfered with observed spectra.

Two color pump-probe experiments were conducted in order to observe the timeresolved electronic spectrum of MK following $400 \mathrm{~nm}$ excitation and are shown in Figure 2.3. Probe beam wavelengths were $511 \mathrm{~nm}$ or $549 \mathrm{~nm}$ and are indicated in the figures. 
Prevalent features of the spectra are similar in all cases and, in fact, the traces in $d_{6^{-}}$ DMSO and $d_{4}-\mathrm{MeOH}$ differ primarily in the timescale of each feature and their relative intensity. Fits were made with exponential functions to determine the timescale associated with individual features.

For all two color pump-probe spectra, immediately following excitation from the $400 \mathrm{~nm}$ pump pulse there is an initial decay in the intensity of the probe beam, indicating the presence of an excited state absorption. It should be noted that in these traces a peak going down from the initial reading (left side) indicates a decrease in probe intensity (transient absorption of the excited state) and a peak going up indicates an increase in probe intensity (stimulated emission of the excited state). This absorption quickly transitions into an emissive state within a few hundred femtoseconds, indicated by an increase in probe beam intensity. The emissive state then decays into a metastable absorptive state. Here is where the two probe wavelengths and solvents differ in timescales.

In $d_{4}-\mathrm{MeOH}$ it takes $\sim 2.75 \mathrm{ps}$ for the emissive state to decay for both probe wavelengths. In $d_{6}$-DMSO, on the other hand, it takes $6 \mathrm{ps}$ for the emissive state to decay in the green and $15 \mathrm{ps}$ for it to decay in the yellow. While not specifically indicated in the figure, it should be noted that $\mathrm{MK}$ in $d_{4}-\mathrm{MeOH}$ the probe beam regains its initial intensity before $50 \mathrm{ps}$, indicating the molecule has returned to its ground state. Traces in $d_{6}$-DMSO show the final state has a much longer lifetime which is not determined here. 


\subsubsection{Computational Results}

Results of geometry optimizations for MK at the DFT level using B3LYP functional and the $6-31+G(d, p)$ basis set indicate a twisted structure in the ground state. In Figure 2.2B it is clearly seen that the two rings are twisted relative to each other. The twist angle of the phenyl rings is found to be $56^{\circ}$. The dipole moment was found to be $5.17 \mathrm{D}$ in the direction of the oxygen atom. In addition, the dimethylamino groups were calculated to be pyramidal and not planar.

Results of Hessian and Raman frequency calculations were compared to experimentally measured ground state spectra. The experimental and theoretical frequencies are shown in Table 2.1. More on the theoretical frequencies will follow in the remaining sections when the experimental values are discussed.

\subsubsection{Ground State Vibrational Spectra of MK}

Ground state fs/ps-CARS spectra of MK in $d_{6}$-DMSO and $d_{4}-\mathrm{MeOH}$ are shown in Figure 2.4. Solvent peaks are indicated with asterisks. Results of theoretical calculations were used to assist in the assignment of measured vibrational modes. Calculations were conducted for the molecule in its gas phase and hence deviation may occur between experimentally observed solution-state spectra and those predicted for the free molecule in gas. In addition, use of density functional theory and the B3LYP functional tend to overestimate vibrational frequencies and a scaling factor is generally used. These factors

are commonly tabulated and can be found on the CCCBDB website. ${ }^{20}$ Here a scaling factor of 0.964 was used. Assignments of observed modes are included in Table 2.1. 
Several representative vibrations are shown in Figure 2.5. The carbonyl peak for this molecule appears at $1630 \mathrm{~cm}^{-1}$ and $1625 \mathrm{~cm}^{-1}$ in $d_{6}$-DMSO and $d_{4}-\mathrm{MeOH}$, respectively. This is in agreement with previous studies on substituted benzophenones. ${ }^{17,21,22}$ The extensive conjugation and charge transfer present in MK are directly responsible for the observed frequency. Charge transfer between the donor and acceptor increases in highly polar solvents leading to an increase in the carbonyl bond length and a resulting decrease in its stretching frequency.

Perhaps the most dramatic feature of MK in both solvents is located in the 1590 $\mathrm{cm}^{-1}$ region. This peak is the most intense ground state mode in this molecule. In $d_{6^{-}}$ DMSO it clearly consists of a single mode centered at $1590 \mathrm{~cm}^{-1}$ while in $d_{4}-\mathrm{MeOH}$ it is centered at $1588 \mathrm{~cm}^{-1}$. This peak is due to the combination mode phenyl $\mathrm{CC}+\mathrm{C}=\mathrm{O}$ stretch. The $1500-1550 \mathrm{~cm}^{-1}$ region contains two peaks in both solvents. The more intense peak near $1540 \mathrm{~cm}^{-1}$ is assigned to the phenyl $8 \mathrm{~b}$ while the peak at $1519 \mathrm{~cm}^{-1}$ in $d_{6}$-DMSO and $1529 \mathrm{~cm}^{-1}$ in $d_{4}-\mathrm{MeOH}$ is assigned to the phenyl $19 \mathrm{~b}$ mode. Also worth mentioning is the $1365 \mathrm{~cm}^{-1}$ mode in $d_{4}-\mathrm{MeOH}$. This mode plays an important role in the excited state evolution of the species in this solvent and is assigned as the pure phenyl-N stretch. ${ }^{10}$

Other modes can also be assigned, including the biphenyl C-C stretch between the phenyl rings and the carbonyl carbon at $1150 \mathrm{~cm}^{-1}$, the phenyl $\mathrm{CC}+$ methyl twist that is present at $1425 \mathrm{~cm}^{-1}$ in $d_{6}$-DMSO, and the pure methyl twisting at 1445 and $1435 \mathrm{~cm}^{-1}$ in $d_{6}$-DMSO and $d_{4}-\mathrm{MeOH}$, respectively. 


\subsubsection{Excited State Vibrational Evolution}

The evolution of the vibrational states of MK in the first excited singlet state, much like the two color pump-probe experiments, indicate the presence of multiple structural conformations. Figure 2.6 contains a contour plot illustrating the temporal evolution of the excited state of MK in $d_{6}$-DMSO. The x-axis is the anti-Stokes scattering frequency, the y-axis is the amount of time elapsed since excitation, and the z-axis is the intensity of the peaks. The ground state contribution has not been removed from this plot, so there are both ground and excited state peaks.

In Figure 2.6 there is a peak at $1500 \mathrm{~cm}^{-1}$ that appears shortly following excitation and experiences a noticeable upshift in frequency. This peak is assigned to the phenyl CC stretch with phenyl-N stretching. Eventually this peak reaches a stable frequency and remains there for the duration of the experiment. This feature was found to remain for greater than 300 ps. Other features in the contour plot do not undergo frequency or intensity changes to any significant extent. The remainder of Figure 2.6 shows the time evolution of the phenyl-N + phenyl-CC stretch.

Figure 2.7 displays the frequency and intensity evolution of the $1500 \mathrm{~cm}^{-1}$ feature. The frequency of this peak upshifts until it reaches a steady value at ten picoseconds and remains there with little change for over $300 \mathrm{ps}$. The intensity profile indicates an unstable intensity during the initial stages of excitation. This is followed by a dramatic increase of the intensity starting at $1.3 \mathrm{ps}$. This increase was fit to an exponential function with a time constant of $2.6 \mathrm{ps}$. There is then a subsequent decay of the peak intensity with an exponential decay of $3.7 \mathrm{ps}$ to reach a stable intensity. The final intensity is constant within experimental error for over 300 ps. 
Additional excited state experiments in DMSO were conducted in the low frequency regime. No ground state modes for MK in this solvent were observed, however, a singular excited state peak was observed near $285 \mathrm{~cm}^{-1}$. This frequency represents the torsional motion of the rings in MK. Dynamics of this peak are shown in Figure 2.8. The actions of this peak are largely in agreement with the phenyl-CC + phenyl-N mode described above. The mode appears at $1.3 \mathrm{ps}$ and increases in frequency until reaching a constant value by 50 ps. The intensity quickly reaches a maximum by 5 ps and gradually decays until completely disappearing by 500 ps.

Excited state vibrational dynamics of $\mathrm{MK}$ in $d_{4}-\mathrm{MeOH}$ are similar to those seen in $d_{6}$-DMSO. The contour plot for $\mathrm{MK}$ in $d_{4}-\mathrm{MeOH}$ is shown in Figure 2.9. This figure also includes a comparison of the ground and excited state spectra of MK in this solvent 100 fs after excitation. This figure details the evolution of MK in its excited state in this solvent. The most dramatic feature is located at $1380 \mathrm{~cm}^{-1}$. This peak appears immediately following excitation and decays very quickly. Another important excited state peak is located at $1500 \mathrm{~cm}^{-1}$. The peaks at 1575 and 1590 are ground state peaks.

The peak dynamics of the $1380 \mathrm{~cm}^{-1}$ peak and the $1500 \mathrm{~cm}^{-1}$ are shown in Figure 2.10. These peaks are assigned as the pure phenyl-N stretch and the combination phenyl $\mathrm{C}-\mathrm{C}+$ phenyl-N stretch, respectively. The pure $\mathrm{Ph}-\mathrm{N}$ mode experiences very fast evolution, reaching its maximum intensity at $100 \mathrm{fs}$ and decaying completely by 1 ps. The frequency of this peak evolves quickly with a timescale shorter than the duration of the pulse.

The phenyl C-C + phenyl-N mode takes longer to evolve. This peak appears shortly after excitation and reaches a maximum intensity by $400 \mathrm{fs}$. Its frequency 
gradually increases throughout the experiment. After 400 fs this peak begins to decay until it merges into its ground state counterpart at $2.5 \mathrm{ps}$ and can no longer be measured.

\subsection{Discussion}

Experiments have been conducted to determine the vibrational evolution of MK in its lowest excited singlet state. Two color pump-probe experiments have been conducted in $d_{4}$-MeOH and $d_{6}$-DMSO using $400 \mathrm{~nm}$ excitation to monitor the change in a probe beam at $511 \mathrm{~nm}$ or $549 \mathrm{~nm}$. These results indicate that immediately following photoexcitation there is a short lived absorptive state that quickly evolves into an emissive state. This emissive state then decays into a long-lived absorptive state.

Two color pump-probe results are in agreement with those of Palit and coworkers who showed in a recent paper using transient absorption measurements the excited state evolution of MK in several solvents. ${ }^{2}$ Their conclusion was that immediately following excitation the FC state undergoes planarization to form the ICT state. This initial state displays a strong transient absorption that is very short lived. The ICT state is emissive and has a longer lifetime than the FC state, consistent with our results. Finally, the planar ICT state experiences a twisting of one dimethylamino group to an angle of 90 degrees with respect to the ring, resulting in the formation of the TICT state which displays a long-lived excited state absorption.

Our excited state vibrational results indicate a complicated evolution of MK in the excited state. They show that upon excitation in $d_{6}$-DMSO the phenyl C-C + phenyl-N mode of MK is significantly altered. Its ground state frequency is $1519 \mathrm{~cm}^{-1}$ in this solvent but in the excited state it begins near $1500 \mathrm{~cm}^{-1}$, experiences an upshift to 1514.7 
$\mathrm{cm}^{-1}$ and remains at this final frequency for over $300 \mathrm{ps}$. This peak indicates a dynamic vibration involving the dimethylaniline moiety that experiences significant changes in the excited state.

The low frequency vibration present at $285 \mathrm{~cm}^{-1}$ represents the torsion of the dimethylaniline groups with respect to the carbonyl. Its appearance comes shortly after excitation and its frequency increases until it stabilizes around 50 ps after excitation. These dynamics indicate a twisting process of the two rings in the excited state. The frequency shift indicates this angle does not stabilize for some time after excitation. Once the frequency stabilizes it remains constant for several hundred picoseconds.

Dynamics in $d_{4}-\mathrm{MeOH}$ help to further understand the actions of $\mathrm{MK}$ in its excited state. The pure phenyl-N mode has a frequency of $1365 \mathrm{~cm}^{-1}$ in the ground state. Immediately following excitation this peak begins to increase in frequency until it reaches a final position and maximum intensity at $1384 \mathrm{~cm}^{-1} 100 \mathrm{fs}$ after excitation. This peak then quickly decays within 1 ps. The phenyl C-C + phenyl-N mode in this solvent behaves in a fashion similar to that in $d_{6}$-DMSO. In the ground state this peak is present at $1529 \mathrm{~cm}^{-1}$ while in the excited state it appears at $1513 \mathrm{~cm}^{-1}$ and gradually increases in frequency until it merges into the corresponding ground state mode and can no longer be discerned.

The vibrational study, when considered in conjunction with transient absorption measurements, helps elucidate the changing structure of MK in its excited state. Immediately following excitation in $d_{4}-\mathrm{MeOH}$ the pure phenyl-N stretch increases in frequency and intensity, and then quickly decays away. This peak is indicative of the planarization of MK in going from the FC to the ICT state and is consistent with the 
initial absorptive state in this solvent and the assignment of a $600 \mathrm{fs}$ lifetime of this state by Palit and coworkers. ${ }^{2}$ The phenyl C-C + phenyl-N stretch appears shortly after excitation and evolves for $2.5 \mathrm{ps}$. This mode is consistent with the production of the ICT state from the FC state.

A more complete picture of MK can be drawn using results in $d_{6}$-DMSO. The phenyl $\mathrm{C}-\mathrm{C}+$ phenyl-N stretch is the only excited state peak observed in the fingerprint region. It appears nearly a picosecond after excitation, indicating it is associated with the ICT state of MK. This mode increases in frequency for several picoseconds, and remains for the duration. The upshift of this mode to reach a stable frequency is indicative of the production of the TICT state because its frequency is constant for very long timescales.

The low frequency mode at $285 \mathrm{~cm}^{-1}$ has been assigned to the torsional motion of the dimethylanilino groups with respect to the carbonyl. This mode shows that there is a twisting process taking place in the $S_{1}$ state of $M K$. The gradual frequency change of this peak indicates that the angle of the phenyl rings relative to one another is changing for some time after excitation. Once in the TICT state the geometry of the molecule is stable for some time and this frequency does not change.

\subsection{Conclusions}

The fs/ps-CARS technique has been used to study the vibrational evolution of MK in its first excited singlet state. DFT calculations are used to assign the ground state vibrational modes of MK. For the first time spectra of the first excited singlet state of MK are obtained in $d_{6}$-DMSO and $d_{4}-\mathrm{MeOH}$. Results in these solvents have shown several interesting vibrational modes which help elucidate structural changes. In $d_{4}-\mathrm{MeOH}$ the 
frequency evolution of the pure phenyl-N and phenyl C-C + phenyl-N modes indicate the planarization of the FC state to produce the ICT state. The evolution of these peaks is consistent with the $\sim 600$ fs timescale attributed by Palit et al to the lifetime of the FC state. The longer timescales associated with the phenyl C-C + phenyl-N and the low frequency torsional mode in $d_{6}$-DMSO indicate the production of the TICT state in this molecule.

\subsection{Acknowledgements}

Financial support from Ames Laboratory and Iowa State University is greatly appreciated. Special thanks go to Professor Mark Gordon and his group for helpful discussions regarding computations.

\subsection{References}

1. Z. R. Grabowski, K. Rotkiewicz, and W. Rettig, Chem. Rev. 103, 3899 (2003).

2. J. A. Mondal, H. N. Ghosh, T. K. Ghanty, T. Mukherjee, D. K. Palit, J. Phys Chem. A 110, 3432 (2006).

3. L. C. T. Schoute, Chem. Phys. Lett. 195, 255 (1992).

4. W. M. Kwok, C. Ma, M. W. George, D. C. Grills, P. Matousek, A. W. Parker, D. Phillips, W. T. Toner and M. Towrie, Photochem. Photobiol. Sci. 6, 987, (2007)

5. S. Arzhantsev, K. Zachariasse, and M. Maroncelli, J. Phys. Chem. A 1103454 (2006).

6. E. van Veldhoven, H. Zhang, W. Rettig, R. G. Brown, J. D. Hepworth, and M. Glasbeek, Chem. Phys. Lett. 363, 189 (2002). 
7. T. P. Shcherban, V. M. Granchak, T. V. Sakhno, and O. A. Khakhel, Theor. Exp. Chem. 41, 198 (2005).

8. T. Pal, M. Paul, and S. Ghosh, THEOCHEM 860, 8 (2008).

9. D. I. Schuster, M. D. Goldstein, and P. Bane, J. Am. Chem. Soc. 99, 187, (1977).

10. C. N. Engert, S. Umpathy, W. Kiefer, and H. Hamaguchi, Chem. Phys. Lett. 218 87 (1994).

11. T. Tahara and H. Hamaguchi, Chem. Lett. 21, 17 (1992).

12. B. D. Prince, A. Chakraborty, B. M. Prince, and H. U. Stauffer, J. Chem. Phys., 125, 044502 (2006).

13. B. D. Prince, A. Chakraborty, B. M. Prince, M. S. Gordon, and H. U. Stauffer, submitted to J. Phys. Chem. A.

14. A. M. Weiner, Rev. Sci. Instrum., 71, 1929 (2000).

15. R. L. McCreery, Raman Shift Frequency Standards; URL; http://www.chemistry.ohio-state.edu/ rmccreer/shift.html, last accessed 6-21-09.

16. M. W. Schmidt, K. K. Baldridge, J. A. Boatz, S. T. Elbert, M. S. Gordon, J. H. Jensen, S. Koseki, N. Matsunaga, K. A. Nguyen, S. J. Su, T. L Windus, M.

Dupuis, J. A. Montgomery, J. Comp Chem 14, 1347 (1993).

17. P. Sett, T. Misra, S. Chattopadhyay, A. K. De, P. K. Mallick, Vib. Spectrosc. 44, 331 (2007).

18. E. Vogel, A. Gbureck, W. Kiefer, J. Mol. Struc. 550-551, 177 (2000).

19. T. Vijayakumar, I. Hubert Joe, C. P. Reghunadhan Nair, V. S. Jayakumar, Chem. Phys., 343, 83 (2008).

20. Computational Chemistry Comparison Benchmark Database; URL; http://cccbdb.nist.gov/, last accessed 6-21-09.

21. V. Volovsek, G. Baranovic, L. Colombo, and J. R. Durig, J. Raman Spectrosc. 22, 35 (1991)

22. T. M. Kolev and B. A. Stamboliyska, Spectrochim. Acta, Part A 56, 119 (1999). 


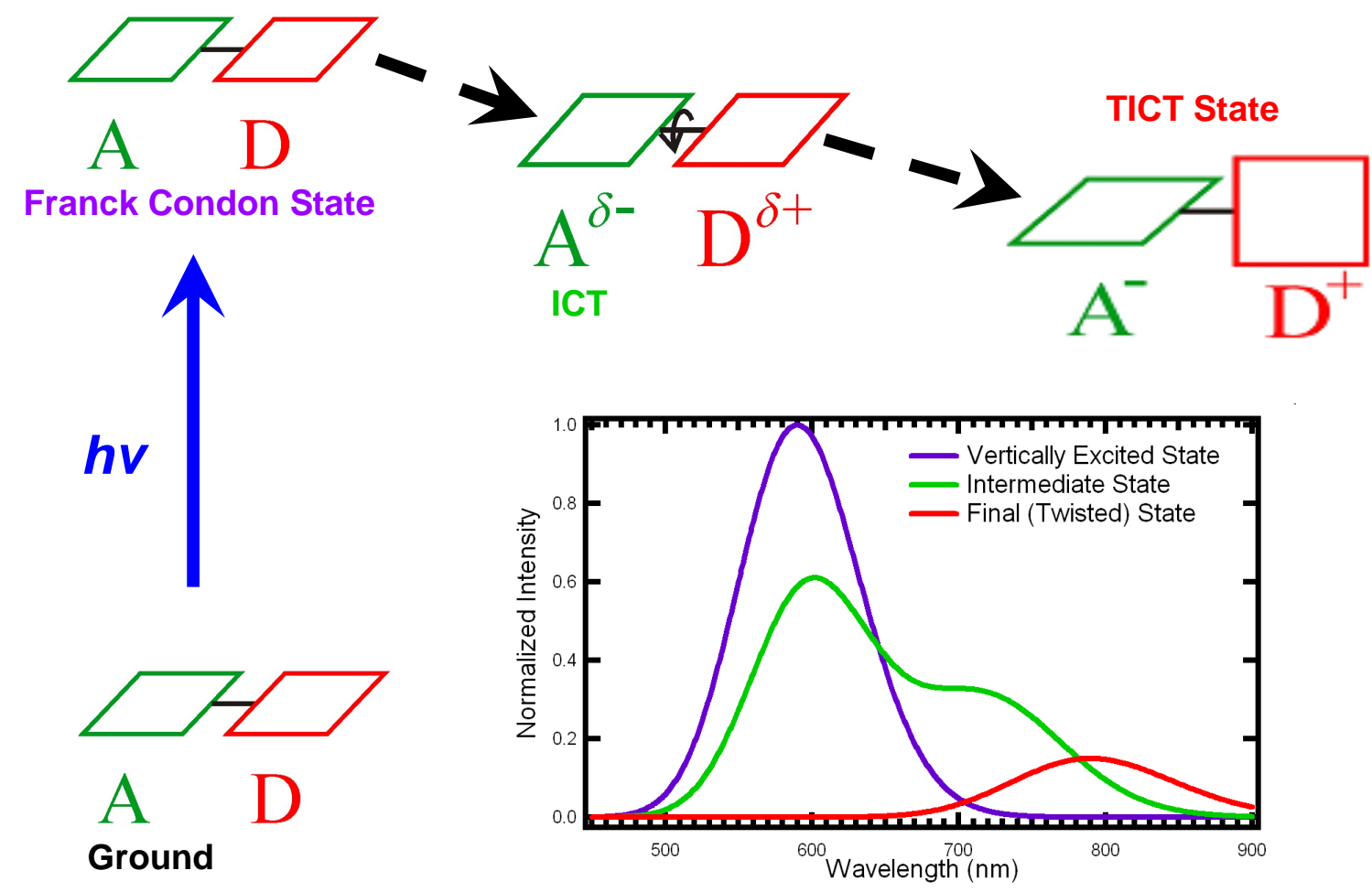

Figure 2.1 - Pictorial description of the differing excited state processes for TICT-type molecules in solvents of differing polarity. These molecules are generally made up of an electron donating group (D) and an electron accepting group (A). The Franck Condon state is the only state accessed in low polarity solvents before fluorescence occurs. In slightly polar solvents a charge separation can occur, leading to a certain proportion of the molecules fluorescing at a higher wavelength from the intramolecular charge transfer state while those that do not undergo charge separation show the FC-type fluorescence. In highly polar solvents a complete transfer of charge can be observed, which is facilitated by the twisting of the donor relative to the acceptor. This results in a large red shift of the fluorescence and a marked decrease in the fluorescence quantum yield.

\section{A}

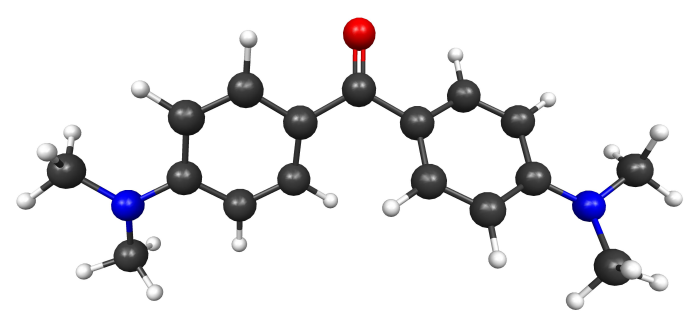

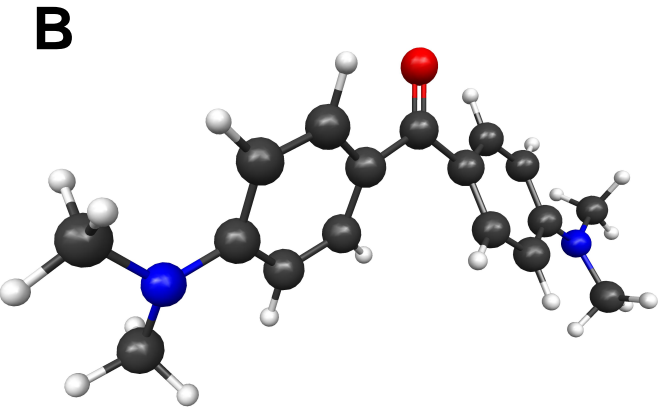

Figure 2.2 - A) Molecular structure of Michler's Ketone. B) The ground state configuration is twisted. 


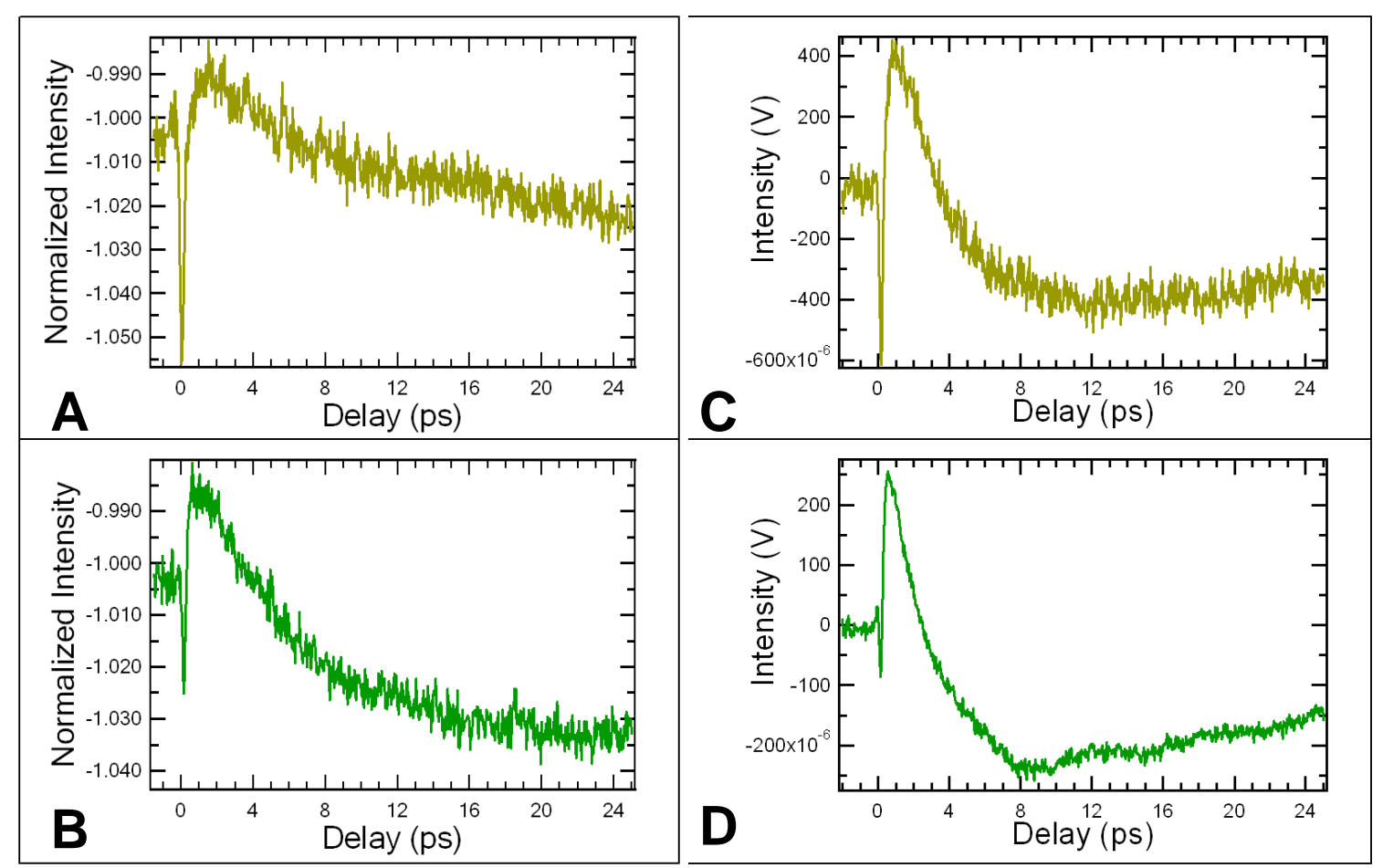

Figure 2.3 - Transient absorption spectra of MK in different solvents. A\&B) Dynamics of the $549 \mathrm{~nm}$ and $511 \mathrm{~nm}$ probe pulses, respectively, following excitation with $400 \mathrm{~nm}$ pulse in $d_{4}-\mathrm{MeOH}$. C\&D) Dynamics of the $549 \mathrm{~nm}$ and $511 \mathrm{~nm}$ probe pulses, respectively, following excitation with $400 \mathrm{~nm}$ pulse in $d_{6}$-DMSO. In each case there is an initial negative-going spike indicating the FC state. This then becomes positive going indicating the presence of the emissive ICT state. Finally each trace shows the long-lived TICT state by long-lived absorptive state. 


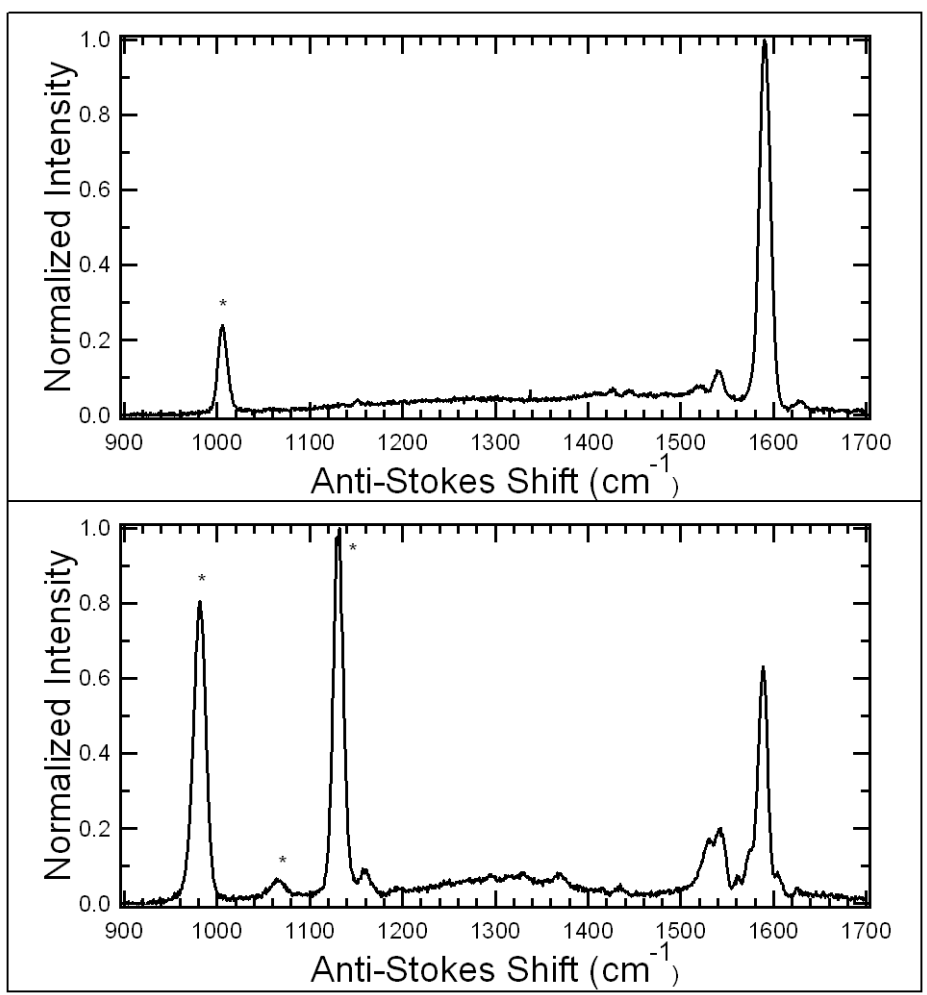

Figure 2.4 - Ground state fs/ps-CARS spectra of MK in top: $d_{6}$-DMSO and bottom: $d_{4}$-MeOH.

\begin{tabular}{|l|l|l|l|l|}
\hline $\begin{array}{l}d_{6}-\mathrm{DMSO} \\
\text { Frequency }\end{array}$ & $\begin{array}{l}d_{4}-\mathrm{MeOH} \\
\text { Frequency }\end{array}$ & $\begin{array}{l}\text { Calculated } \\
\text { Frequency }\end{array}$ & $\begin{array}{l}\text { Scaled } \\
\text { Frequency }\end{array}$ & Assignment \\
\hline 1630 & 1625 & 1712 & 1650 & $\mathrm{C}=\mathrm{O}$ \\
\hline 1590 & 1588 & 1660 & 1600 & $\mathrm{Ph} \mathrm{CC}+\mathrm{C}=\mathrm{O}$ \\
\hline 1540 & 1543 & 1595 & 1537 & $\mathrm{Ph} \mathrm{CC}$ \\
\hline 1519 & 1529 & 1563 & 1506 & Ph CC + Ph-N \\
\hline 1445 & 1435 & 1500 & 1445 & Methyl Twist \\
\hline N/A & 1365 & 1389 & 1339 & Ph-N Stretch \\
\hline 1425 & N/A & 1475 & 1422 & PhCC + Methyl Twist \\
\hline 1150 & 1158 & 1174 & 1132 & Biphenyl CC Stretch \\
\hline
\end{tabular}

Table 2.1 - Frequency assignments for MK ground state. 


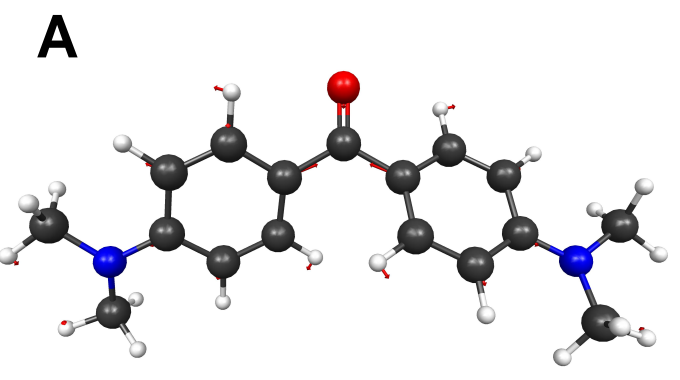

B
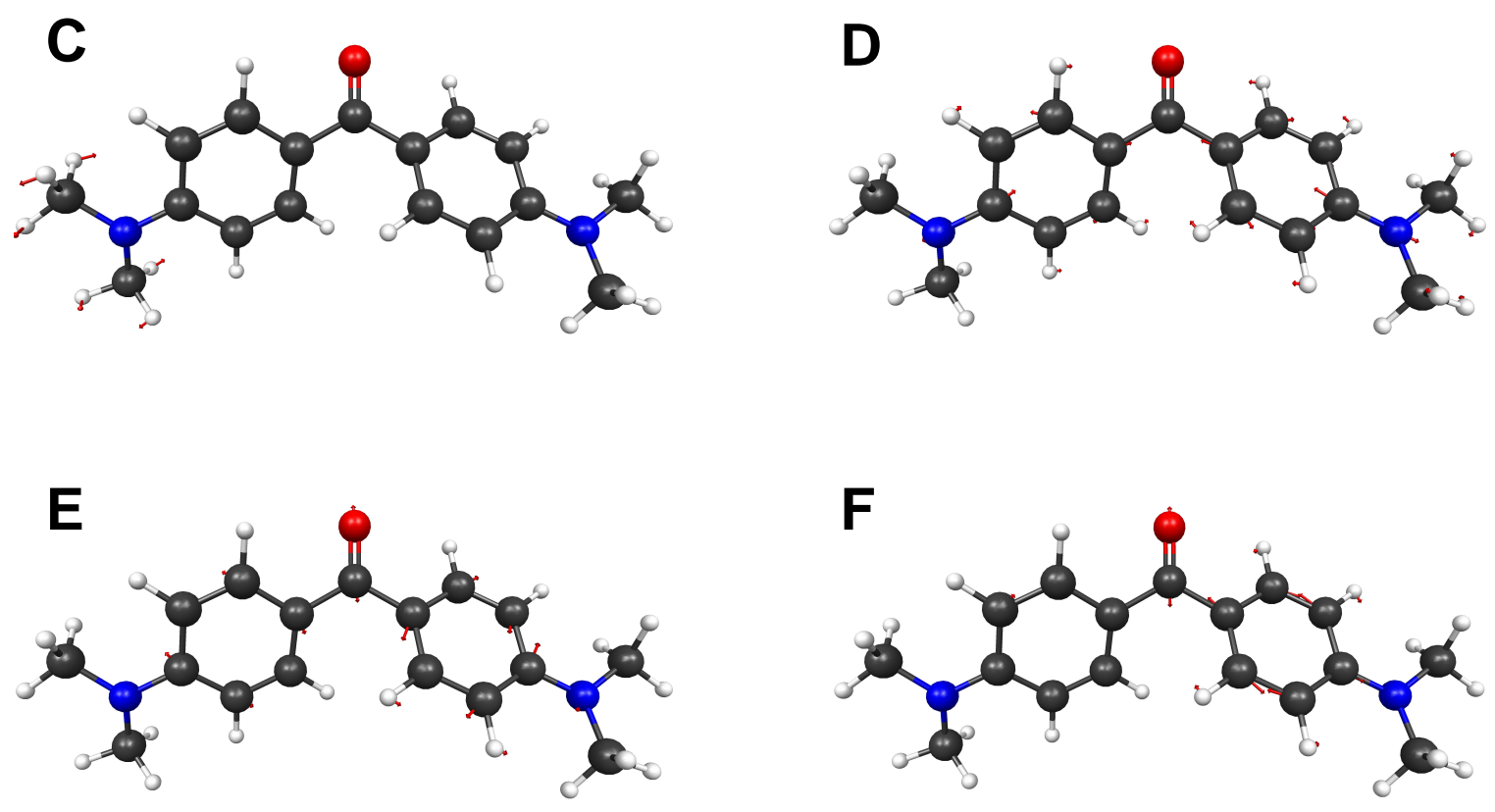

Figure 2.5 - Representative vibrations of MK. A) $1150 \mathrm{~cm}^{-1}$, B) $1380 \mathrm{~m} \mathrm{~cm}^{-1}$, C) $1425 \mathrm{~cm}^{-1}$, D) $1520 \mathrm{~cm}^{-1}$ E) $1540 \mathrm{~cm}^{-1}$ F) $1590 \mathrm{~cm}^{-1}$ 


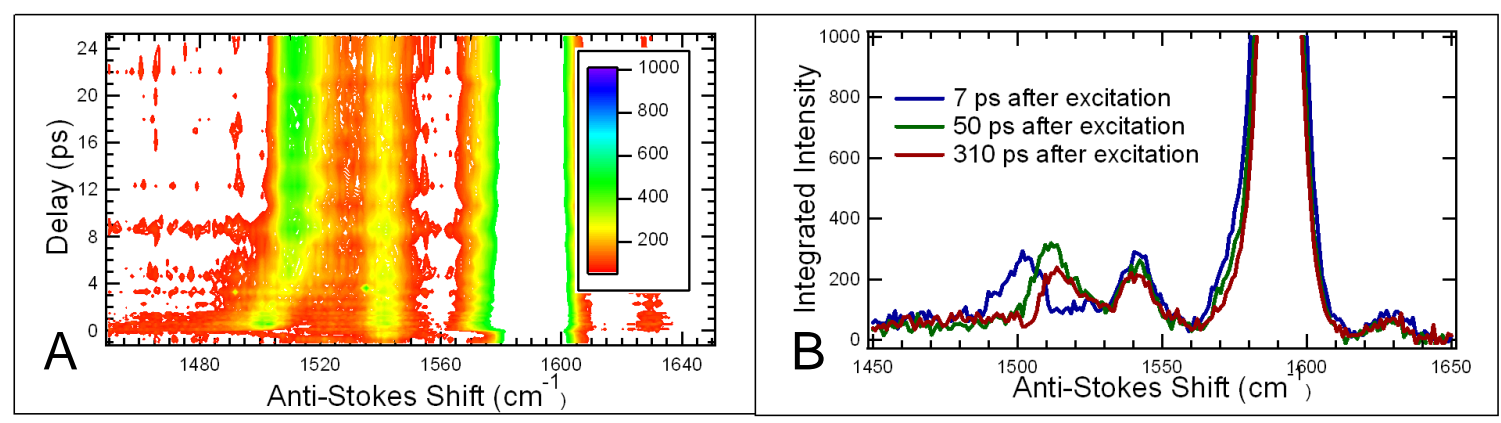

Figure 2.6 - A) Excited state + ground state contour plot of MK in $d_{6}$-DMSO. Time evolves along the yaxis so that every horizontal slice is a spectrum at a specific time delay. B) Excited + ground state spectra at several time delays following excitation. These panels clearly indicate the evolution of the $\mathrm{Ph}-\mathrm{CC}+\mathrm{Ph}-\mathrm{N}$ mode.

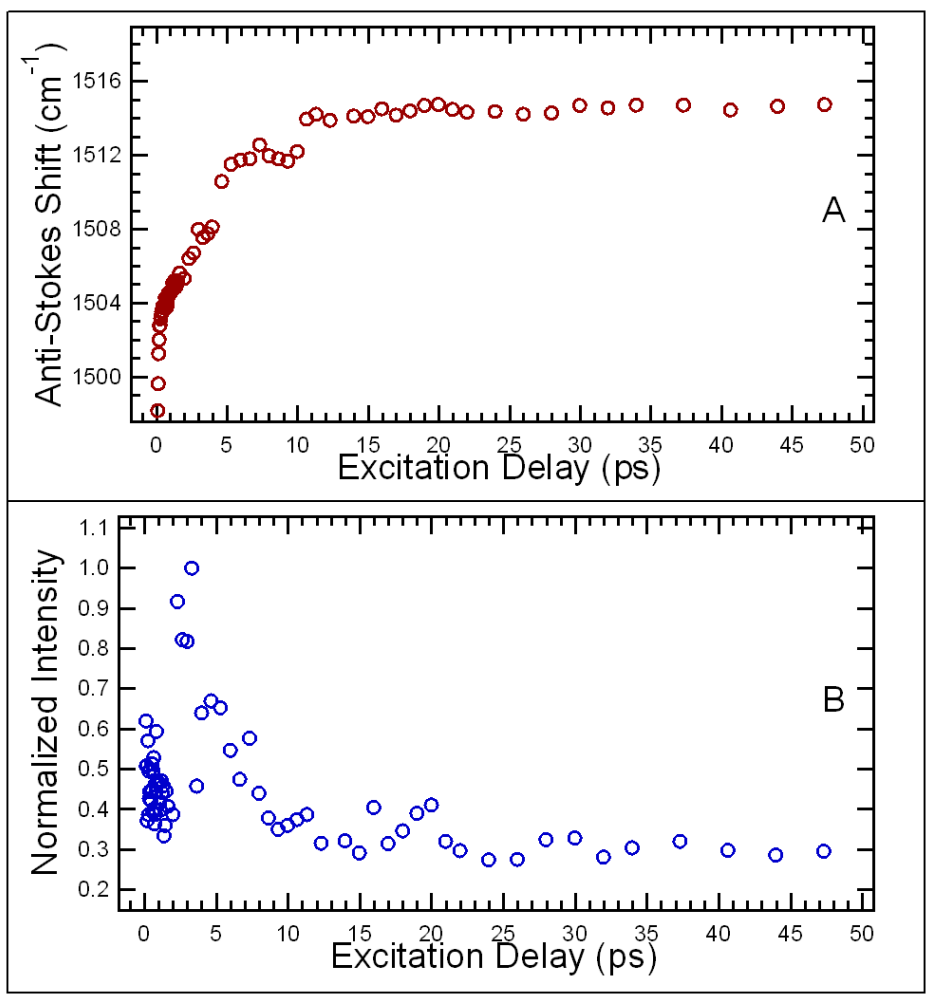

Figure 2.7 - Evolution of the Ph-CC + Ph-N mode. A) The frequency gradually upshifts until reaching a steady value by $20 \mathrm{ps}$. B) The intensity of this mode increases in the first $5 \mathrm{ps}$ and then becomes steady around 20 ps after excitation. 


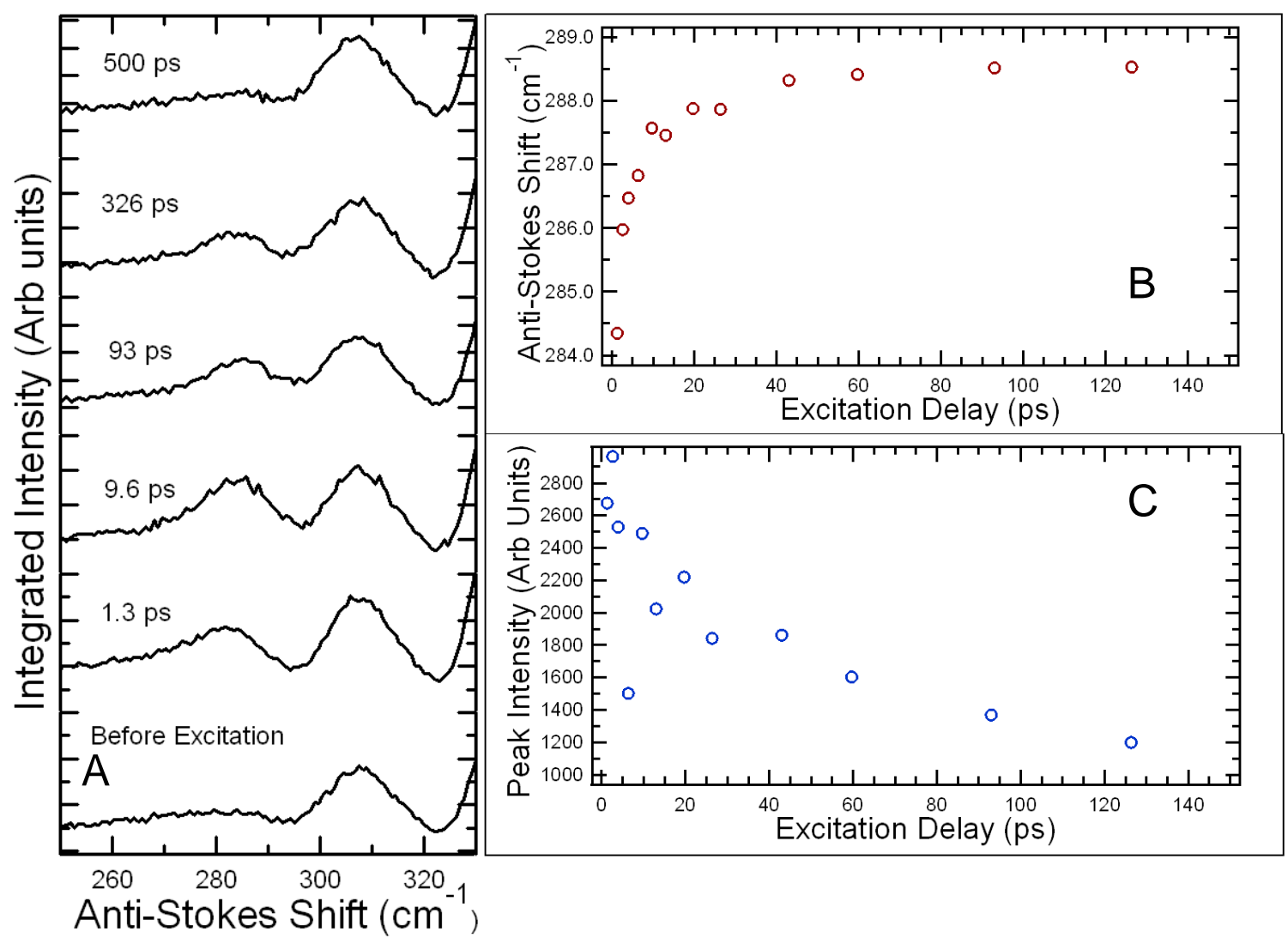

Figure 2.8 - Low frequency dynamics. A) Excited state spectra in the region of interest. B) Frequency evolution of the torsional mode. C) Intensity evolution of the torsional mode. 


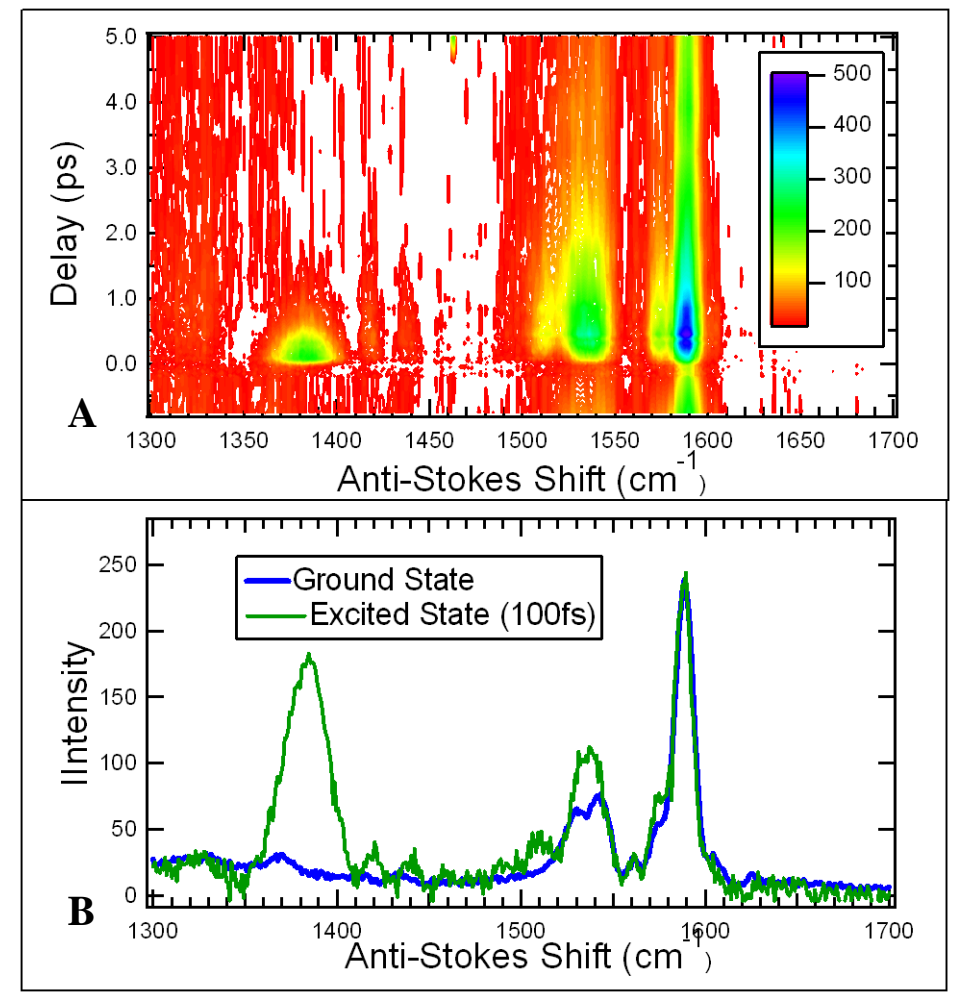

Figure 2.9 - Excited state dynamics in $d_{4}-\mathrm{MeOH}$. A) Contour plot showing dynamical change of the pure phenyl-N mode. B) Excited state spectrum at 100 fs overlaid with the ground state spectrum to indicate the change in vibrational structure.
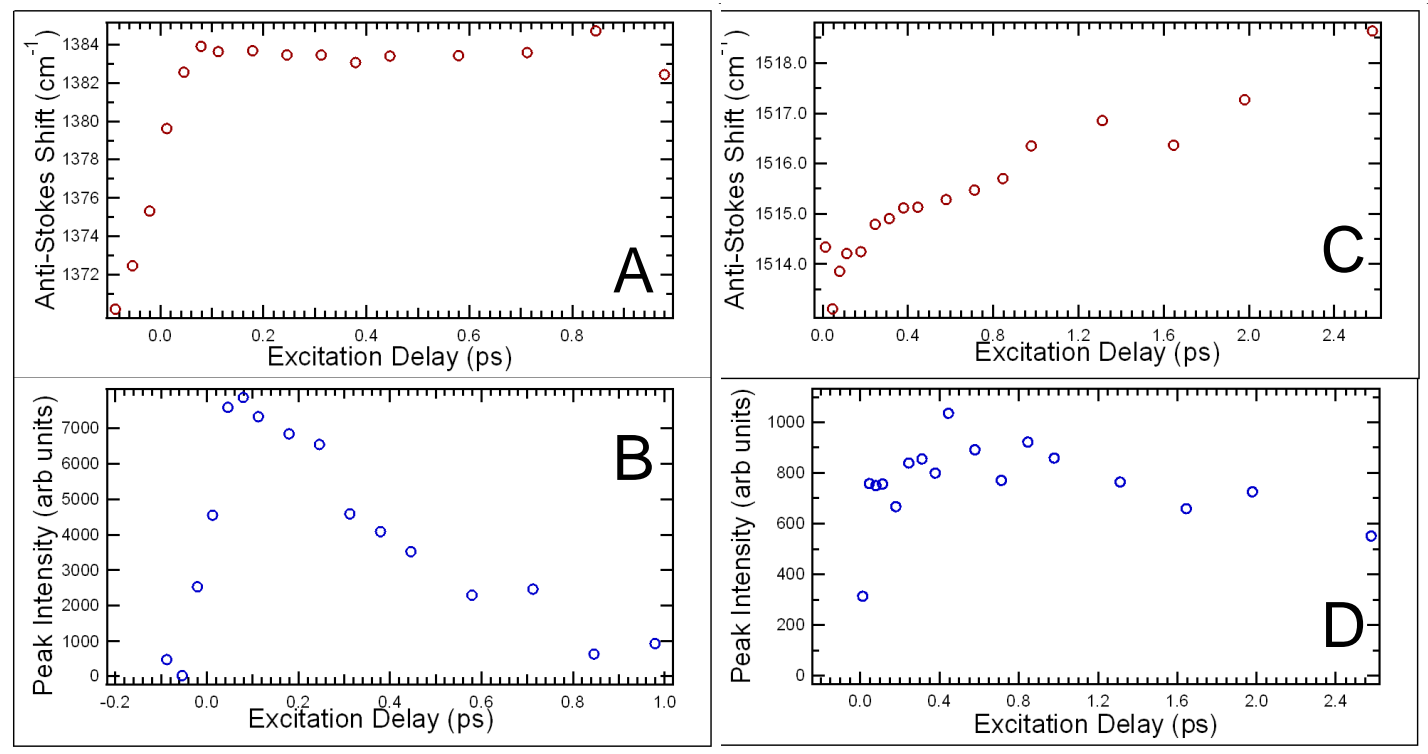

Figure 2.10 - Peak dynamics in $d_{4}-\mathrm{MeOH}$. A) Frequency shift of the phenyl-N mode. B) Intensity shift of the phenyl-N mode. C) Frequency change of the phenyl-N + phenyl-CC mode and D) its corresponding intensity dynamics. 


\title{
CHAPTER 3: ANALYSIS OF THE EFFECT OF SOLVATION ON THE VIBRATIONAL STRUCTURE OF COUMARIN 120 USING AN EXPERIMENTAL AND THEORETICAL APPROACH
}

\author{
Alex J. Blom, Hans U. Stauffer, and Mark S. Gordon
}

\subsection{Abstract}

The vibrational structure of Coumarin 120 is studied using a combined experimental and theoretical approach. Density functional theory calculations are used to assign frequencies observed in FTIR and spontaneous Raman experiments. Solvation effects on the geometry of Coumarin 120 and its frequencies are then studied using coherent anti-Stokes Raman spectroscopy in conjunction with calculations employing the polarizable continuum model. It is found that the carbonyl stretching frequency decreases in solvents with high dielectric constant, consistent with its increase in bond length in such solvents. A mode attributed to the $\mathrm{C}-\mathrm{N}$ deformation undergoes an increase in frequency in solvents of high dielectric constant, indicating a shortening of this bond and a planarization of the amino group.

\subsection{Introduction}

The 7-amino-4-methylcoumarins are used in a wide variety of applications. They were first developed for use as laser dyes and have found work as solvation probes,

antimicrobial drugs, and as reaction sensitizers. ${ }^{1-3}$ Coumarin $120(\mathrm{C} 120)$ is the simplest of the 7-amino-4-methylcoumarins and is shown in Figure 3.1. This molecule consists of a 
benzopyrone moiety with amino (7) and methyl (4) substituents and has been studied experimentally and theoretically by several groups. ${ }^{4-12}$ The solvatochromism displayed by this dye makes it an intriguing molecule to study.

C120, unlike its more rigid counterpart, coumarin 153, has freedom of motion of its amino group. In solvents of differing polarity the structure of this group can change to become more energetically stable. It has been suggested that the ground state structure of C120 is heavily influenced by solvent polarity. ${ }^{6,8,12}$ In particular, it has been theorized that a gradual charge transfer occurs from the amino group to the carbonyl as solvent polarity is increased. Recent calculations support this statement but the effect of solvent on the vibrational structure of C120 has not, to this point, been analyzed.

In this paper a combined theoretical and experimental approach is adopted to better understand the vibrational structure of C120 in its ground state. FTIR and spontaneous Raman spectroscopy are combined with density functional theory (DFT) calculations to assign experimentally observed vibrational frequencies. For calculations the B3LYP functional is used with the 6-311G(d,p) basis set. This combination has been shown to yield molecular geometries and harmonic frequencies with good accuracy and low computational cost relative to other methods like MP2 and coupled cluster., ${ }^{2,6,13}$ Calculations are also conducted using the solvation models, specifically the polarizable continuum model (PCM), and are combined with coherent Raman experiments to determine how the frequencies of C120 are affected upon solvation. 


\subsection{Methods}

\subsubsection{Experimental}

Infrared spectra were obtained using an FTIR spectrometer (Nicolet Nexus 470) in the range from $650 \mathrm{~cm}^{-1}$ to $4000 \mathrm{~cm}^{-1}$. The spectral resolution was set to $1 \mathrm{~cm}^{-1}$ and 128 spectra were recorded. Peaks were assigned using the OMNIC software suite from Thermo Scientific.

Spontaneous Raman spectra were obtained using a $785 \mathrm{~nm}$ Renishaw diode laser coupled to a Nikon TE-2000-U wide field optical microscope operated in confocal mode. The incident beam is focused to the sample using a 40x oil immersion objective that is also used to collect Raman scatter. The signal is then separated using a dichroic mirror and focused onto a $100 \mu \mathrm{m}$ pinhole before being sent into a Kaiser Holospec $1.8 \mathrm{i}$ spectrometer. A notch filter removes any Rayleigh scatter and the signal then passes through a $100 \mu \mathrm{m}$ slit. The spectrum is collected using a Princeton Instruments 400BR IR-enhanced CCD array that is controlled using Winspec. The spectra were calibrated using a 50/50 (v/v) toluene/acetonitrile standard.

Condensed phase Spectra of C120 were taken using the coherent anti-Stokes Raman scattering method termed fs/ps-CARS. This experimental method allows for multiplexed detection of vibrational spectra. ${ }^{14}$ Details of this setup have been described previously. Briefly, $50 \mathrm{fs}$ pulses are generated at $800 \mathrm{~nm}$ using a Ti:Sapphire laser system operating at $1000 \mathrm{~Hz}$ and approximately $2 \mathrm{~mJ} /$ pulse. The fundamental is divided into several portions, the first of which is sent into an optical parametric amplifier (OPA) to produce broadband ultrafast pulses. The output of the OPA is recombined with the fundamental to generate two pulses in the visible region. Typical pulse frequencies and 
widths are: $\omega_{1}=19570 \mathrm{~cm}^{-1}[511 \mathrm{~nm}]$; bandwidth $\Delta \omega=300 \mathrm{~cm}^{-1}$ and $\omega_{2}=18210 \mathrm{~cm}^{-1}$ [549 $\mathrm{nm}] ; \Delta \omega=350 \mathrm{~cm}^{-1}$. Another portion of the fundamental is sent into a grating stretcher-compressor pulse shaper to generate narrowband picosecond duration pulses $\left(\sim 10 \mathrm{~cm}^{-1}\right) .{ }^{15}$ Use of an adjustable slit allows the pulse bandwidth to be changed depending on experimental considerations. Polarization of all beams was set to parallel.

The three beams generated in the experimental setup are directed into the sample with a folded BOXCARS geometry. This allows the signal beam to be spatially separated from the three input beams. The signal is then focused into a spectrometer and spectrally dispersed. An Ocean Optics USB-2000 handheld spectrometer was used for these experiments. This spectrometer allows for $6 \mathrm{~cm}^{-1}$ resolution. ${ }^{14}$

\subsubsection{Materials}

All materials were used as received. 7-amino-4-methylcoumarin (Coumarin 120), was obtained from Exciton. For fs/ps-CARS experiments the dye was dissolved in dimethyl sulfoxide (DMSO, certified ACS) which was acquired from Fisher. Concentrations of the dye in DMSO ranged from 30-50 mM. For FTIR experiments the dye was ground with $\mathrm{KBr}$ powder (Fisher IR-grade) and then compressed into a pellet. Spontaneous Raman experiments were conducted using C120 powder.

\subsubsection{Density Functional Theory Calculations}

Molecular simulations for isolated C120 were conducted at the DFT level of theory using the B3LYP functional and the 6-311G(d,p) basis set. All calculations were conducted using the GAMESS computational chemistry software. ${ }^{16}$ Following geometry 
optimizations, Hessian runs were conducted to confirm that an optimized geometry had been reached and to calculate the harmonic frequencies associated with the molecule. The infrared and Raman intensity of the vibrations were found using the default electric field strengths in GAMESS. Optimized structures and vibrational motions of C120 were visualized using MacMolPlt. ${ }^{17}$

To determine how the vibrational structure of C120 is affected upon solvation, geometry optimizations and Hessians were conducted in the presence of a dielectric field using the polarizable continuum model (PCM) at the same level of theory and basis set as the gas phase calculations. This model places a bulk solvent around the chromophore and considers the solvent molecules as a large featureless entity characterized by its dielectric constant. The solute can thus perturb the solvent and vice versa until an optimal structure is reached. Several solvents were used as indicated in the text. The conductor-like PCM (C-PCM) model was chosen for these studies. ${ }^{9,18}$

\subsection{Results and Discussion}

\subsubsection{Optimized Structure}

The optimized structure of C120 in the gas phase is shown in Figure 3.1. The

benzene and lactone rings display the near planarity typical of this class of molecules., ${ }^{2,12}$ The calculations predict a pyramidalization of the amine group to a dihedral angle of $19.88^{\circ}$ with respect to the plane of the benzopyrone moiety. Table 3.1 contains the optimized coordinates for isolated C120. The dipole moment of the ground state is found to be $6.37 \mathrm{D}$ directed toward the carbonyl. 
Additional structural information is included in Table 2.2 which presents a comparison of the calculated geometric parameters of C120 to the crystal structure determined by Jasinski and Woudenberg. ${ }^{12}$ It should be noted that the calculations in this paper were performed on a single C120 molecule while the crystal structure contains structural data for C120 in a solid matrix and thus the geometric parameters may be slightly different. For these studies it was not computationally feasible to perform optimizations with multiple C120 molecules present.

The calculated bond lengths and angles are found to be in good agreement with the experimentally determined crystal structure. The DFT method using the B3LYP functional tends to do a good job of predicting bond lengths and this is confirmed for C120. ${ }^{9,13}$ Additionally, the bond angles are within $1 \%$ error in most cases. The largest error in the calculations involves the oxygen atoms of the ester functionality and $\mathrm{sp}^{3}$ hybridized hydrogens. X-ray diffraction studies showed the crystal structure to include hydrogen bonding of the carbonyl with hydrogens on neighboring molecules. Since the calculations were performed on a single molecule, the lengths and angles of the previously mentioned bonds are expected to be different than the crystal.

\subsubsection{Vibrations of $\mathrm{C120}$}

Experimental FTIR spectra for C120 are shown in Figure 3.2. The top panel displays the range $650-3700 \mathrm{~cm}^{-1}$ The bottom panel displays the carbonyl and vibrations lower in frequency $\left(650-1700 \mathrm{~cm}^{-1}\right)$. Figure 3.3 displays the experimental spontaneous Raman spectrum in the $650-1700 \mathrm{~cm}^{-1}$ region. Figure 3.4 displays FTIR and Raman results on a single chart so vibrations of both methods may be more easily correlated. 
The carbonyl peaks in the two different experimental methods differ greatly in intensity. Most molecules show a very strong IR absorption for the carbonyl peak. The Raman intensity of the carbonyl stretch is much lower than the IR intensity and is often not observed. Additionally, the experimental setup of the Raman microscope trails off in efficiency near the carbonyl region. For the spontaneous Raman spectrum in Figure 3.3 this mode is not readily evident. There is, however, a small peak at $1677 \mathrm{~cm}^{-1}$ that is assigned to this vibration.

Table 3.3 contains peak frequencies and their assignments for C120. Figure 3.5 shows the numbering scheme for vibrations of the molecule. The benzene ring is indicated by B while the lactone ring is indicated by L. Ring vibrations specific to each ring are indicated with their corresponding letter. All calculated frequencies in this paper are raw, unscaled values. In the table it is seen that the carbonyl mode and high frequency ring modes in the $1500-1600 \mathrm{~cm}^{-1}$ region are overestimated by $2-4 \%$ while modes lower in frequency than this are spot on. The carbonyl is overestimated by $8.3 \%$. Since most of the modes observed are calculated with such low error and the ring modes that are overestimated can still be correlated to experimental findings, the calculated frequencies are left unscaled.

When appropriate (and possible) ring motions are assigned using the notation established by Wilson. ${ }^{19}$ These assignments are possible if each ring clearly displays the motions indicated in Wilson's paper. Most of these ring modes account for motions of the substituents inherently. In certain cases the assignments in Table 3.3 indicate motion of the substituents in addition to the ring modes in order to emphasize a particular 
displacement. These mainly involve the phenyl-N bond $\left(\mathrm{C}_{7}-\mathrm{N}\right)$ and the carbonyl bond $(\mathrm{C}=\mathrm{O})$. Motions of the methyl carbon relative to the ring are indicated by 'methyl.'

The carbonyl stretch in the IR at $1680 \mathrm{~cm}^{-1}$ displays some interaction with the high frequency ring modes, the closest of which is assigned at $1621 \mathrm{~cm}^{-1}$. This causes the $\mathrm{C}=\mathrm{O}$ stretch to appear very broad. The ring modes near $1600 \mathrm{~cm}^{-1}$ are very closely spaced and assignment of the individual peaks was not straightforward. The best fit of the frequencies is shown in the table. The Raman spectrum in this region displays a strong peak at $1598 \mathrm{~cm}^{-1}$ with shoulders on the high $\left(1610 \mathrm{~cm}^{-1}\right)$ and low $\left(1585 \mathrm{~cm}^{-1}\right)$ frequency sides.

Assignments for the ring modes in the $1600 \mathrm{~cm}^{-1}$ region are largely in agreement with those of similar molecules. ${ }^{2}$ The mode at $1621 \mathrm{~cm}^{-1}$ in the IR and $1610 \mathrm{~cm}^{-1}$ in the Raman is assigned to the $8 \mathrm{~b}$ stretch of the benzene ring combined with carbonyl stretching, $\mathrm{C}_{3}-\mathrm{C}_{4}$ stretch, $\mathrm{C}_{7}-\mathrm{N}$ stretch and the amino group scissoring motion. The peak near $1600 \mathrm{~cm}^{-1}$ is the most intense feature of both the IR and Raman and is assigned to the simultaneous $8 \mathrm{~b}$ stretching of both rings.

Other interesting features include the doublet near $1550 \mathrm{~cm}^{-1}$. This mode has an interesting shape in the IR with a tail extending on the low frequency side. In the Raman this feature was easily fit using a sum of two lorentzian functions. The high frequency feature $\left(1544 \mathrm{~cm}^{-1} \mathrm{IR}, 1557 \mathrm{~cm}^{-1}\right.$ Raman $)$ is assigned to the $8 \mathrm{a}$ stretch of the benzene ring, which has an inherent component of the $\mathrm{C}_{7}-\mathrm{N}$ stretch, combined with the $\mathrm{C}_{3}-\mathrm{C}_{4}$ stretch, which results in the $\mathrm{C}_{4}$-Methyl bond being deformed. The low frequency mode at 1541 $\mathrm{cm}^{-1}$ in the IR and $1550 \mathrm{~cm}^{-1}$ in the Raman is the $19 \mathrm{a}$ motion of the benzene ring. This motion results in the $\mathrm{C}_{7}-\mathrm{Nand} \mathrm{C}_{\mathrm{X}}-\mathrm{C}_{4}$ stretches since these are substituents in this motion. 
The dramatic peak at $1165 \mathrm{~cm}^{-1}$ in the Raman (also present in IR) indicates the motion associated with the 14 mode of the lactone ring. This motion includes the asymmetric stretching of the C-O-C portion of the ring. The low frequency side of this peak at $1150 \mathrm{~cm}^{-1}$ indicates the $\mathrm{C}-\mathrm{H}$ stretching of the benzene ring along with the $\mathrm{C}_{5}-\mathrm{C}_{\mathrm{Y}}$ stretch. This motion includes the asymmetric stretching of the $\mathrm{C}-\mathrm{O}-\mathrm{C}$ portion of the ring. Additionally this mode contains the $\mathrm{C}-\mathrm{H}$ bending motion of the benzene ring. Below $1000 \mathrm{~cm}^{-1}$ the peak near $690 \mathrm{~cm}^{-1}$ is clear in both the IR and Raman spectrum. This mode is the 6 a motion of both rings.

Figure 3.5 shows several representative modes for $\mathrm{C} 120$. These modes have been selected because they are seen in both the experimental IR and Raman data. Relative atomic displacements are indicated by arrows. Assignments for these modes can be found in Table 3.3.

\subsection{3 - Solvation effects on C120 structure}

The suggestion that solvent properties may affect the structure of C120 implies that its vibrational characteristics should be highly sensitive to solvent environment. ${ }^{7-9}$ The dielectric constant of a solvent, which is a rough measure of its polarity, is expected to exhibit a great influence on the geometry and thus the vibrations of C120. Condensedphase spectra of C120 in DMSO have been taken using the fs/ps-CARS method. The PCM model has been added in to calculations to account for solvation effects on C120. Solvents utilized included aprotic solvents of varying polarity. Since the PCM model cannot account for specific interactions such as hydrogen bonds, these are left to future studies. 
As mentioned previously, it has been suggested that the amino group is able to transfer charge to the carbonyl under polar conditions. Figure 3.7 illustrates the resonance structure that would then be present. If a charge transfer does occur the length of the carbonyl bond is expected to increase, which would cause the stretching motion of this bond to decrease in frequency. The other consequence of charge transfer would be the shortening of the phenyl-N bond and the gradual planarization of the amino group. Table 3.4 analyzes how these two bonds are affected upon inclusion of different solvents into the geometry optimization.

The data in Table 3.4 clearly indicate that the ground state structure of C120 is affected by solvation. A reliable indicator of the charge transfer in polar solvents is the increase of the dipole moment of C120 in going from nonpolar to slightly polar, to highly polar solvents. This indicates a charge separation between the amino and carbonyl groups, causing an increase in the dipolar character of the molecule. Structurally, as the solvent dielectric constant increases, the length of the carbonyl bond begins to increase, going from $1.207 \AA$ in cyclohexane to $1.211 \AA$ in carbon tetrachloride, and finally to $1.214 \AA$ in the highly polar solvents acetone, nitromethane, and DMSO. At the same time the length of the phenyl-N bond gradually decreases with increasing dielectric constant, as does the dihedral angle between the amino group and the plane of the benzopyrone moiety. Finally, the $\mathrm{NH}_{2}$ bond angle increases to over $114^{\circ}$ which indicates it is becoming less pyramidal and more planar.

Table 3.5 contains the calculated vibrational frequencies for $\mathrm{C} 120$ using PCM to model a continuum solvent. It is seen that many of the modes decrease with increasing solvent dielectric constant, especially the carbonyl mode and major ring modes (1500- 
$1650 \mathrm{~cm}^{-1}$ ). The $1374 \mathrm{~cm}^{-1}$ (in the gas phase) vibration, attributed to have a $\mathrm{C}_{7}-\mathrm{N}$ deformation component, increases in frequency as solvent dielectric constant is increased. This increase in frequency is in line with the decrease of the $\mathrm{C}_{7}-\mathrm{N}$ bond length.

The mode at $1374 \mathrm{~cm}^{-1}$ is the closest to a pure C-N vibration that is observed in this molecule. It is expected that this is why its frequency increases in high polarity solvents. Many of the other modes listed in Table 3.5 have a small component of $\mathrm{C}-\mathrm{N}$ stretch or deformation associated with them, but not enough to cause the frequency of the vibration to increase. This is to say that the molecule in its entirety has a slightly different structure in going from gas phase to nonpolar solvent and eventually to highly polar solvents and this affects each vibration differently. If there were a pure $\mathrm{C}-\mathrm{N}$ stretch as there is a pure $\mathrm{C}=\mathrm{O}$ stretch, it would be easier to determine how the frequency of this vibration changed upon solvation.

Figure 3.8 contains the ground state spectrum of C120 in DMSO acquired using the fs/ps-CARS method. Three peaks were observed including the carbonyl at $1671 \mathrm{~cm}^{-1}$, and two ring modes at $1606 \mathrm{~cm}^{-1}$ and $1621 \mathrm{~cm}^{-1}$, respectively. These experimentally observed modes are assigned to the $8 b(B)+8 b(L)$ and $8 b(B)+C=O \& C_{7}-N \& C_{3}-C_{4}$ Stretch $+\mathrm{NH}_{2}$ Scissor, respectively. To this point spectra have been acquired in only one solvent. The decrease in frequency with respect to the solid state spectrum indicates the carbonyl is influenced by DMSO upon solvation, as shown in the results of PCM calculations. Acquisition of spectra in more solvents would further understanding of the solvation of C120. 


\subsection{Conclusions}

The vibrational structure of $\mathrm{C} 120$ has been determined and its characteristic frequencies assigned through the use of FTIR and Raman spectroscopy in conjunction with DFT calculations. The ground state geometry was found to be in good agreement with the experimentally observed geometry of the C120 crystal. Continuum solvation calculations suggest that there is a charge transfer from the amino group to the carbonyl group in polar solvents, resulting in a lengthening of the $\mathrm{C}=\mathrm{O}$ bond and a concomitant decrease of its vibrational frequency. These calculations also indicate a planarization of the amino group and a shortening of the $\mathrm{C}_{7}-\mathrm{N}$ in polar solvents that results in an increase of the $\mathrm{C}_{7}-\mathrm{N}$ deformation.

\subsection{Acknowledgements}

We would like to thank Kris McKee and Justin Valenstein for use of instruments. Alex would like to thank Sarom Sok, Luke Roskop and Ben Prince for useful discussions. Financial support from Ames Laboratory and Iowa State University is much appreciated.

\subsection{References}

1. X. Liu, M. Dong, X. Chen, M. Jiang, and J. Zhou, Appl. Microbiol. Biotechnol. 78, 241 (2008).

2. E. Vogel, A. Gbureck, and W. Kiefer, J. Mol Struc. 550-551, 177 (2000).

3. M. L. Horng, J. A. Gardecki, and M. Maroncelli, J. Phys. Chem. A 99, 17311 (1995).

4. K. Ando, J. Chem. Phys. 107, 4585 (1997).

5. V. K. Sharma, P. D. Saharo, N. Sharma, R. C. Rastogi, S. K. Ghoshal, and D. Mohan, Spectrochim. Acta, Part A 59, 1161 (2003). 
6. Y. Wang, G. Wu, Acta Phys. Chim. Sin. 23, 1831 (2007).

7. R. J. Cave, K. Burke, and W. Castner, Jr, J. Phys. Chem. A. 1069294 (2002).

8. H. Pal, S. Nad, M. Kumbhakar, J. Chem. Phys. 119, 443 (2003).

9. W. Zhao, L. Pan, W. Bian, and J. Wang, Chem. Phys. Chem., 9, 1593 (2008).

10. A. Fischer, C. Cremer, and E. H. K. Stelzer, Appl. Opt. 34, 1989 (1995).

11. M. Nowakowska, M. Smoluch, and D. Sendor, J. Inclusion Phenom. Macrocyclic Chem. 40, 213 (2004).

12. J. P. Jasinski and R. C. Woudenberg, Acta Cryst, Part C 50, 1954 (1994)

13. C. J. Cramer, Essentials of Computational Chemistry: Theories and Models; Wiley, 2002.

14. B. D. Prince, A. Chakraborty, B. M. Prince, and H. U. Stauffer, J. Chem. Phys., 125, 044502 (2006).

15. A. M. Weiner, Rev. Sci. Instrum., 71, 1929 (2000).

16. M. W. Schmidt, K. K. Baldridge, J. A. Boatz, S. T. Elbert, M. S. Gordon, J. H. Jensen, S. Koseki, N. Matsunaga, K. A. Nguyen, S. J. Su, T. L Windus, M. Dupuis, J. A. Montgomery, J. Comp Chem 14, 1347 (1993).

17. B. M. Bode and M. S. Gordon, J. Mol. Graphics Mod., 16, 133 (1998).

18. M. Cossi, N. Rega, G. Scalmani, and V. Barone, J. Comput. Chem. 24, 669 (2003).

19. E. B. Wilson, Phys. Rev. 45, 706 (1934). 


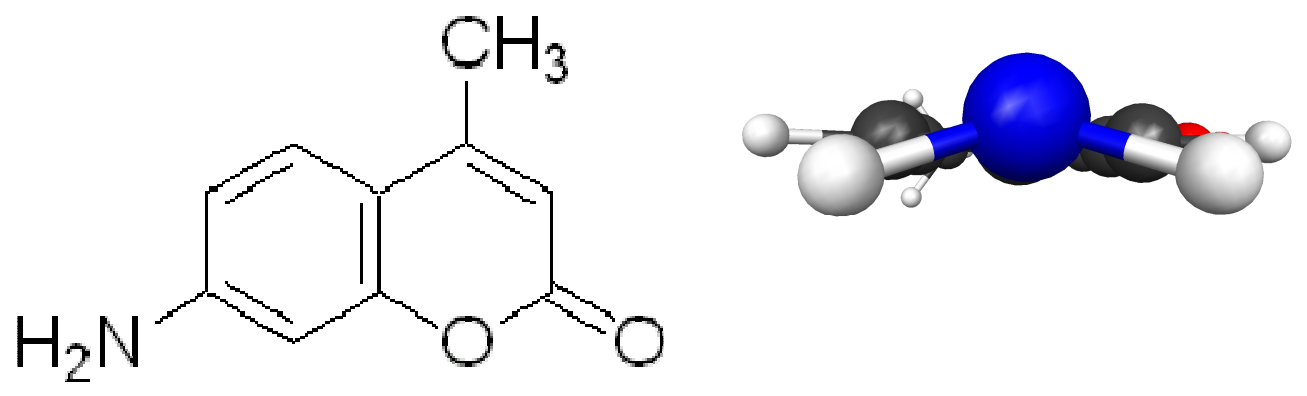

Figure 3.1 - Molecular structure of C120. Black spheres indicate carbon atoms, white is for hydrogen, red for oxygen, and blue for nitrogen. The fused ring core of the molecule is planar but while the amino group displays a pyramidal configuration.

\begin{tabular}{|ccccc|}
\hline Atom & $\begin{array}{c}\text { Atomic } \\
\text { Number }\end{array}$ & $\mathbf{X}(\mathbf{\AA})$ & $\mathbf{Y}(\AA)$ & $\mathbf{Z}(\mathbf{A})$ \\
\hline $\mathrm{C}$ & 6.0 & 0.616135 & -0.204723 & 0.462497 \\
$\mathrm{C}$ & 6.0 & 0.499236 & 1.176850 & 0.549086 \\
$\mathrm{C}$ & 6.0 & -0.418530 & -1.011154 & -0.053200 \\
$\mathrm{C}$ & 6.0 & -1.588008 & -0.353852 & -0.480158 \\
$\mathrm{C}$ & 6.0 & -0.672173 & 1.808713 & 0.118224 \\
$\mathrm{C}$ & 6.0 & -1.721062 & 1.017539 & -0.401067 \\
$\mathrm{O}$ & 8.0 & 1.785446 & -0.742137 & 0.905217 \\
$\mathrm{C}$ & 6.0 & 2.036602 & -2.122373 & 0.878705 \\
$\mathrm{O}$ & 8.0 & 3.098018 & -2.499491 & 1.296342 \\
$\mathrm{C}$ & 6.0 & 0.970407 & -2.947051 & 0.341717 \\
$\mathrm{C}$ & 6.0 & -0.206403 & -2.441483 & -0.107341 \\
$\mathrm{C}$ & 6.0 & -1.276162 & -3.346992 & -0.655003 \\
$\mathrm{H}$ & 1.0 & -1.522822 & -3.083208 & -1.688162 \\
$\mathrm{H}$ & 1.0 & -0.951715 & -4.387694 & -0.635586 \\
$\mathrm{H}$ & 1.0 & -2.198156 & -3.264986 & -0.070508 \\
$\mathrm{H}$ & 1.0 & -2.631672 & 1.496505 & -0.744576 \\
$\mathrm{~N}$ & 7.0 & -0.782659 & 3.187045 & 0.146183 \\
$\mathrm{H}$ & 1.0 & -1.715183 & 3.570026 & 0.126221 \\
$\mathrm{H}$ & 1.0 & 1.331638 & 1.742710 & 0.950824 \\
$\mathrm{H}$ & 1.0 & 1.179605 & -4.008930 & 0.323032 \\
$\mathrm{H}$ & 1.0 & -2.407849 & -0.937317 & -0.881556 \\
$\mathrm{H}$ & 1.0 & -0.156048 & 3.681678 & 0.762183 \\
\hline
\end{tabular}

Table 3.1 - Optimized gas phase geometry for C120 using DFT B3LYP and 6-311G(d,p). 


\begin{tabular}{|c|cc|c|c|}
\hline Bond & DFT $\mathbf{( \AA )}$ & $\begin{array}{c}\text { Exp } \\
\mathbf{( A )}^{\mathbf{1 2}}\end{array}$ & \multicolumn{1}{c|}{ Diff } & \%Error \\
\hline C=O & 1.2013 & 1.22 & -0.0166 & -1.37 \\
O1-C2 & 1.4031 & 1.37 & 0.0321 & 2.35 \\
O1-C4 & 1.3609 & 1.38 & -0.0210 & -1.53 \\
C7-N & 1.3830 & 1.37 & 0.0140 & 1.03 \\
C2-C3 & 1.4509 & 1.42 & 0.0269 & 1.89 \\
C3-C4 & 1.3572 & 1.35 & 0.0062 & 0.46 \\
CX-C4 & 1.4469 & 1.44 & 0.0069 & 0.49 \\
C4-ME & 1.5047 & 1.50 & 0.0097 & 0.65 \\
C5-C6 & 1.3801 & 1.37 & 0.0151 & 1.11 \\
C5-C10 & 1.4078 & 1.41 & 0.0018 & 0.13 \\
C6-C7 & 1.4127 & 1.41 & 0.0067 & 0.48 \\
C7-C8 & 1.3989 & 1.39 & 0.0079 & 0.57 \\
C8-CY & 1.3892 & 1.37 & 0.0152 & 1.11 \\
CX-CY & 1.4095 & 1.39 & 0.0165 & 1.19 \\
N-H & 1.0080 & 0.85 & 0.1580 & 18.59 \\
ME-H & 1.0824 & 0.97 & 0.1124 & 11.59 \\
\hline Angles & & & & \\
\hline CX-O1-C2 & 122.4177 & 121.80 & 0.6177 & 0.51 \\
O=C2-O1 & 117.4104 & 115.20 & 2.2104 & 1.92 \\
O1-C2-C3 & 115.7569 & 117.50 & -1.7430 & -1.48 \\
O=C2-C3 & 126.8325 & 127.30 & -0.4674 & -0.37 \\
C2-C3-C4 & 123.2211 & 122.60 & 0.6211 & 0.51 \\
C3-C4-CX & 118.8772 & 118.90 & -0.0227 & -0.02 \\
C3-C4-C12 & 120.8419 & 120.70 & 0.1419 & 0.12 \\
CX-C4-C12 & 120.2807 & 120.40 & -0.1192 & -0.10 \\
C6-C5-CX & 121.7791 & 122.00 & -0.2208 & -0.18 \\
C5-C6-C7 & 120.3968 & 120.80 & -0.4031 & -0.33 \\
N-7-C6 & 120.4142 & 121.10 & -0.6857 & -0.57 \\
N-C7-C8 & 120.7168 & 120.40 & 0.3168 & 0.26 \\
C6-C7-C8 & 118.8131 & 118.50 & 0.3131 & 0.26 \\
C7-C8-CY & 120.0295 & 119.40 & 0.6295 & 0.53 \\
O1-CY-C8 & 116.4069 & 115.60 & 0.8069 & 0.70 \\
O1-CY-CX & 121.5866 & 120.70 & 0.8866 & 0.73 \\
C8-CY-CX & 122.0063 & 123.70 & -1.6936 & -1.37 \\
C4-CX-C5 & 124.8858 & 125.80 & -0.9141 & -0.73 \\
C4-CX-CY & 118.1392 & 118.50 & -0.3607 & -0.30 \\
\hline Dihedrals & & & & \\
\hline O=C2-O1-CY & -179.7482 & -177.90 & -1.84821 & 1.04 \\
O=C2-C3-C4 & 179.7474 & 178.60 & 1.1474 & 0.64 \\
\hline & & & & \\
\hline
\end{tabular}

Table 3.2 - Comparison of calculated and experimentally determined geometries of C120. Experimental values taken from Ref 12. 

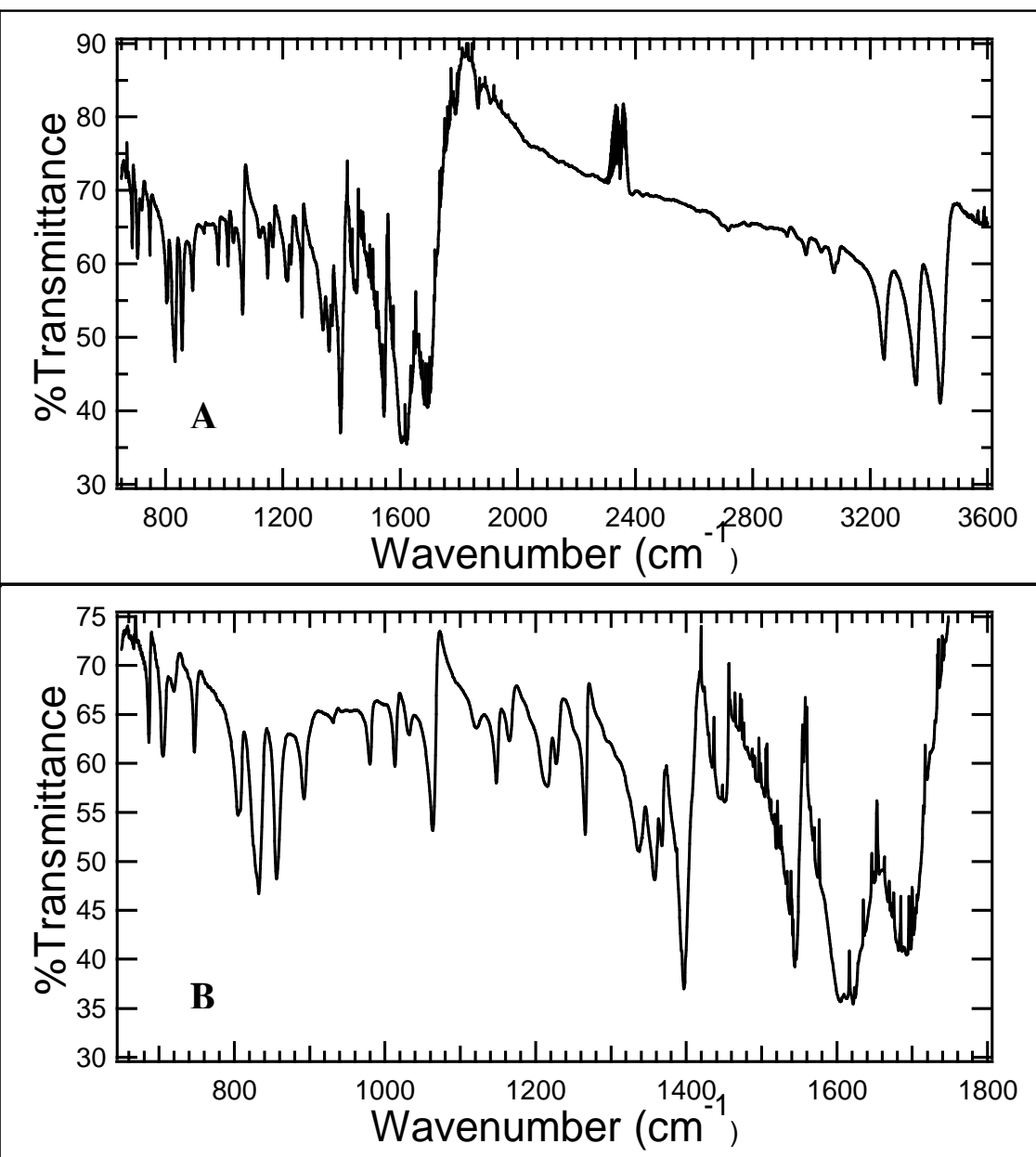

Figure 3.2 - Experimental FTIR spectra for $\mathrm{C} 120 \mathrm{KBr}$ pellet A) Near infrared spectrum in the region from $650-3600 \mathrm{~cm}^{-1}$. B) Near infrared spectrum in the region from $650-1800 \mathrm{~cm}^{-1}$.

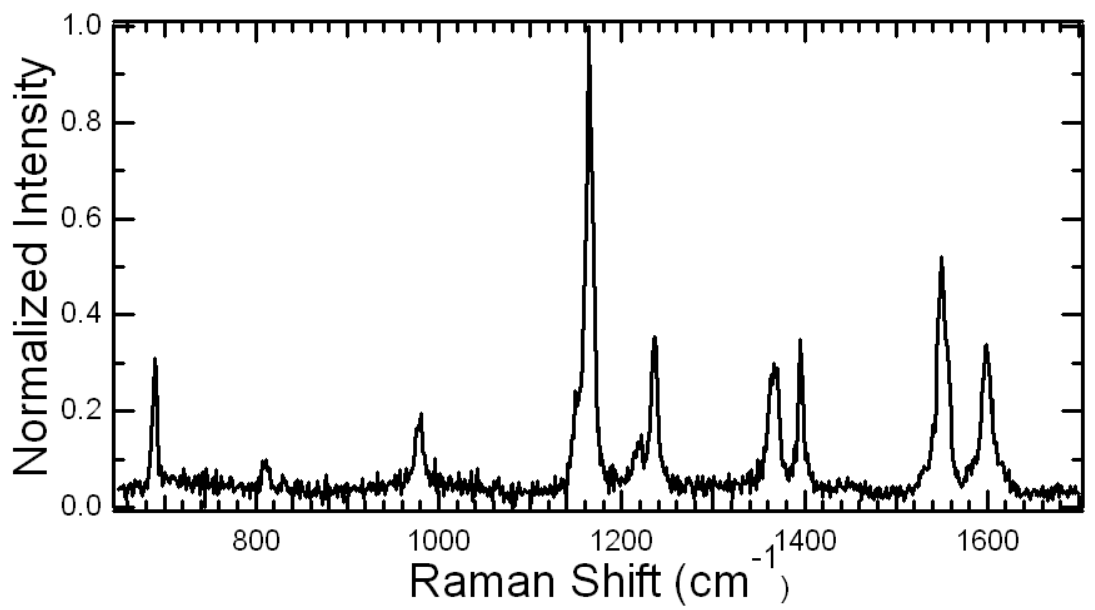

Figure 3.3 - Experimental spontaneous Raman spectrum of C120 powder. 


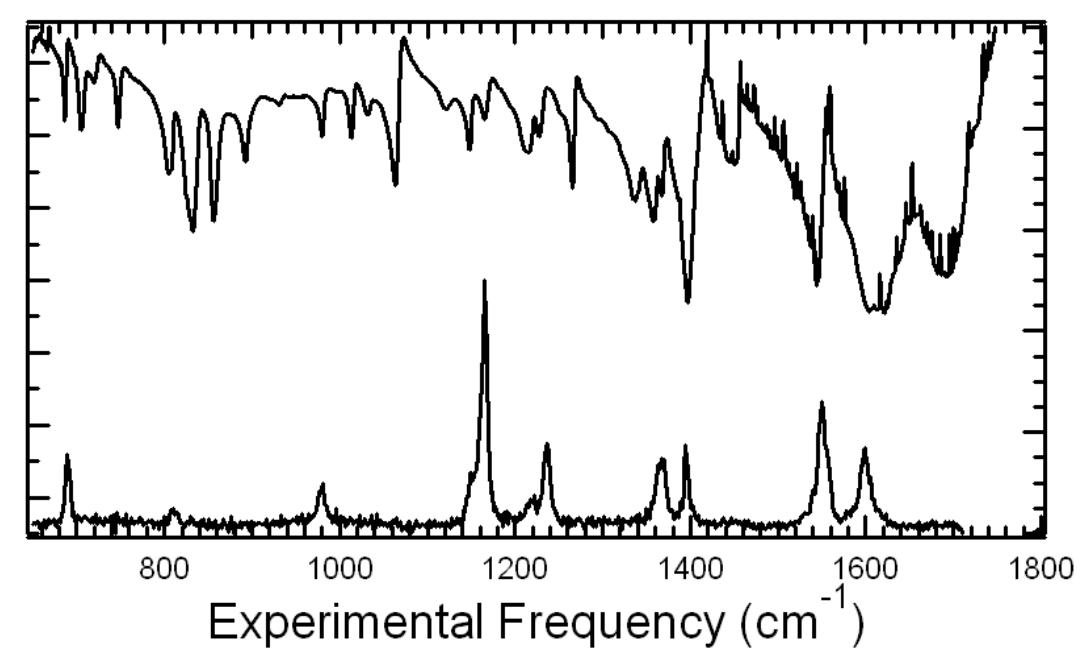

Figure 3.4 - FTIR and Raman spectra of C120.

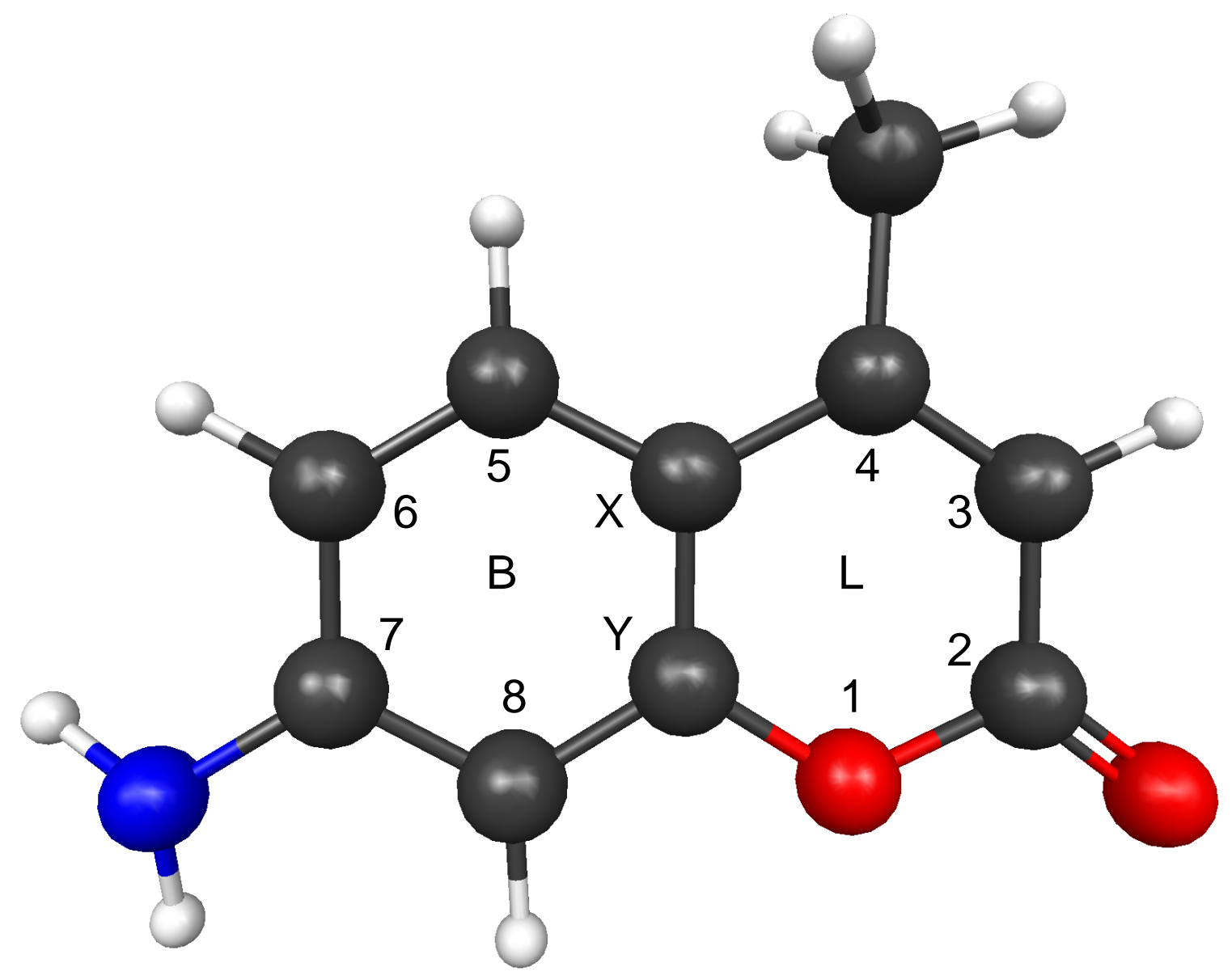

Figure 3.5 - Numbering scheme for C120. Benzene is B, Lactone is L. 


\begin{tabular}{|c|c|c|c|}
\hline $\begin{array}{l}\mathbf{V}_{\mathbf{I R}} \\
\left(\mathrm{cm}^{-1}\right)\end{array}$ & $\begin{array}{l}\mathbf{V}_{\text {Raman }} \\
\left(\mathrm{cm}^{-1}\right)\end{array}$ & $\begin{array}{l}\mathbf{v}_{\text {Calc }} \\
\left(\mathrm{cm}^{-1}\right)\end{array}$ & Assignment \\
\hline 687 & 690 & 692 & $6 a(B)+6 a(L)$ \\
\hline 705 & & 706 & $\mathrm{O}-\mathrm{C}-\mathrm{O}$ Wag $+\mathrm{CH}$ Wag $(\mathrm{L})+4(\mathrm{~B})$ \\
\hline 720 & & 725 & $18 \mathrm{a}(\mathrm{B})$ \\
\hline 747 & & 744 & $4(B)+4(L)$ \\
\hline 806 & 811 & 802 & $17 \mathrm{~b}(\mathrm{~B})$ \\
\hline 808 & & 810 & $12(\mathrm{~L})+6 \mathrm{a}(\mathrm{B})+\mathrm{C}_{7}-\mathrm{N} \mathrm{Str}$ \\
\hline 833 & 830 & 849 & $\mathrm{CH}$ wag $(\mathrm{B}) \mathrm{OOP}$ \\
\hline 856 & & 865 & $\mathrm{C}_{3}-\mathrm{H}$ OOP Wag $+\mathrm{C}=\mathrm{O}$ Wag \\
\hline 892 & & 866 & $\mathrm{C}_{2}-\mathrm{O}$ Str $+\mathrm{CH}$ Bend $(\mathrm{L})$ Ring Breathing (B) \\
\hline 931 & & 936 & $\mathrm{C}_{5} \mathrm{H} \& \mathrm{C}_{6} \mathrm{H}$ OOP bend \\
\hline 980 & 978 & 987 & $\mathrm{C}_{\mathrm{X}}-\mathrm{O}$ Bend $+\mathrm{CH}$ Bend $(\mathrm{B})+\mathrm{C}_{6}-\mathrm{H}$ Str \\
\hline 1013 & & 1023 & 12(B) $+12(\mathrm{~L})$ Methyl Twist \\
\hline 1032 & & 1060 & $\mathrm{C}_{4}$-Methyl Out of plane \\
\hline 1063 & & 1071 & Methyl Rock $+\mathrm{C}=\mathrm{O}$ Bend \\
\hline 1121 & & 1108 & $\mathrm{NH}_{2}$ Twist $+\mathrm{CH}$ Bend $(\mathrm{B})$ \\
\hline 1165 & 1165 & 1146 & $\begin{array}{l}\text { 14(L) [Composed of C-O-C Asymmetric Stretch }+\mathrm{C}_{3}-\mathrm{C}_{2} \text { Str] }+ \\
\mathrm{CH} \text { Bend }(\mathrm{B})\end{array}$ \\
\hline 1148 & 1151 & 1175 & $\mathrm{C}_{6}-\mathrm{H} \& \mathrm{C}_{5}-\mathrm{H}$ bend $+\mathrm{C}_{5}-\mathrm{C}_{Y}$ Stretch \\
\hline 1215 & 1217 & 1227 & C-O-C asymmetric stretch $+18 \mathrm{~b}(\mathrm{~B})+\mathrm{C}_{7}-\mathrm{N}$ Str \\
\hline 1227 & 1236 & 1244 & $\mathrm{C}_{4}$-Methyl Str $+\mathrm{CH}$ Bend $(\mathrm{B} \& \mathrm{~L})+\mathrm{C}_{\mathrm{X}}-\mathrm{C}_{4}$ Stre \\
\hline 1266 & & 1282 & $\mathrm{CH}$ Bend $(\mathrm{B})+19 \mathrm{a}(\mathrm{L})$ \\
\hline 1336 & & 1351 & $13(\mathrm{~B})$ \\
\hline 1358 & 1364 & 1374 & $14(\mathrm{~B})+\mathrm{C}_{7}-\mathrm{N}$ Deform \\
\hline 1367 & 1370 & 1404 & $\begin{array}{l}\mathrm{C}_{4} \text {-Methyl Deform + Methyl symmetric bend }+\mathrm{C}_{X}-\mathrm{C}_{4} \text { stretch }+ \\
\mathrm{CH} \text { Bend }(\mathrm{L})\end{array}$ \\
\hline 1380 & & 1423 & $\mathrm{C}_{4}$-Methyl Str \\
\hline 1397 & 1396 & 1475 & $19 b(B)+19 b(L)$ \\
\hline 1436 & 1437 & 1484 & $\mathrm{CH}_{3}$ Twist \\
\hline 1446 & 1446 & 1491 & $\mathrm{CH}_{3}$ Twist $+\mathrm{C}_{3}-\mathrm{C}_{4}$ Stretch \\
\hline 1541 & 1550 & 1544 & $19 a(B)+C_{7}-N+C_{x}-C 4$ \\
\hline 1544 & 1557 & 1585 & $8 \mathrm{a}(\mathrm{B})+\mathrm{C}_{7}-\mathrm{N}$ deform $+\mathrm{C}_{3}-\mathrm{C}_{4} \mathrm{Str}+\mathrm{C}_{4}$-Methyl Deform \\
\hline 1585 & 1580 & 1653 & $\mathrm{C}_{4}-\mathrm{C}_{3} \mathrm{Str}+\mathrm{C}=\mathrm{O} \mathrm{Str}+\mathrm{NH}_{2}$ Scissor $+8 \mathrm{a}(\mathrm{B})$ \\
\hline 1604 & 1598 & 1656 & $8 b(B)+8 b(L)$ \\
\hline 1621 & 1610 & 1670 & $8 b(B)+C=O \& C_{7}-N \& C_{3}-C_{4}$ Stretch $+\mathrm{NH}_{2}$ Scissor \\
\hline 1680 & 1677 & 1818 & $\mathrm{C}=\mathrm{O}$ Stretch \\
\hline
\end{tabular}

Table 3.3 - Experimental and theoretically calculated vibrational frequencies for C120 including assignments. The table only includes calculated frequencies that correspond to experimentally observed frequencies. Others are present but are not shown. Any questions regarding these modes or the ones not experimentally observed should be directed to the author. 

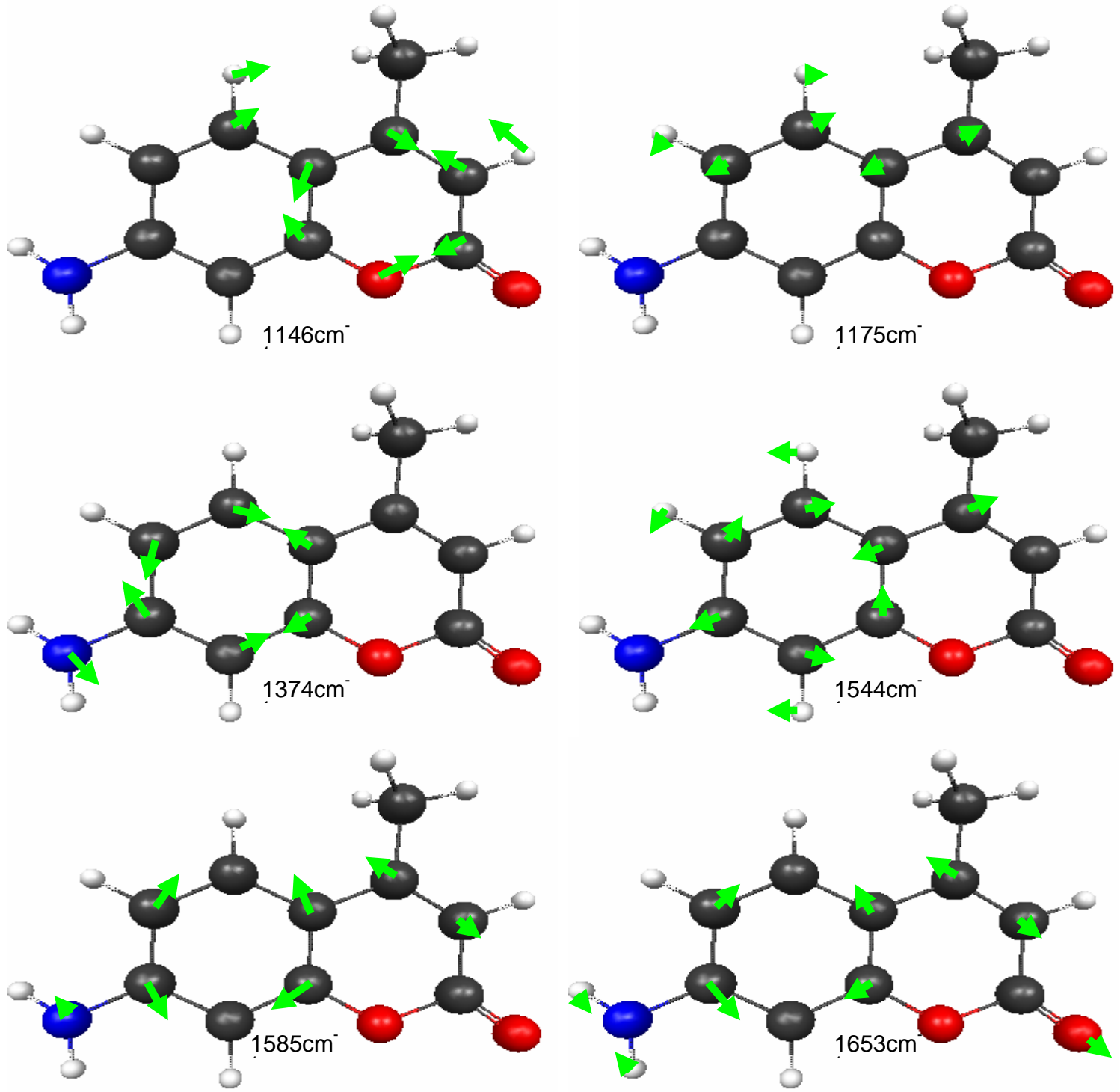

Figure 3.6 - Representative vibrations of the C120 molecule. Unscaled frequencies indicated on the diagram. 


\begin{tabular}{|c|c|c|c|c|c|c|}
\hline Solvent & $\begin{array}{l}\text { Dielectric } \\
\text { Constant }\end{array}$ & $\begin{array}{l}\text { Calculated } \\
\text { Dipole } \\
\text { moment } \\
\text { (D) }\end{array}$ & $\begin{array}{l}\text { Carbonyl } \\
\text { Bond } \\
\text { Length } \\
(\AA)\end{array}$ & $\begin{array}{c}\text { C8-C7-N- } \\
\text { H } \\
\text { Dihedral } \\
\left({ }^{\circ}\right)\end{array}$ & $\begin{array}{l}\text { Ph-N } \\
\text { Bond } \\
\text { length } \\
(\AA)\end{array}$ & $\begin{array}{c}\mathrm{NH2} \\
\text { bond } \\
\text { angle } \\
\left({ }^{0}\right)\end{array}$ \\
\hline $\mathrm{CH}$ & 2.02 & 7.569 & 1.207 & 19.435 & 1.379 & 113.695 \\
\hline $\mathrm{CCl} 4$ & 2.23 & 7.707 & 1.207 & 19.281 & 1.379 & 113.722 \\
\hline Tol & 2.37 & 7.793 & 1.208 & 19.251 & 1.379 & 113.744 \\
\hline $\mathrm{CHCl} 3$ & 4.9 & 8.554 & 1.211 & 18.727 & 1.376 & 113.888 \\
\hline THF & 7.58 & 8.850 & 1.212 & 18.502 & 1.375 & 113.959 \\
\hline ACETONE & 20.7 & 9.227 & 1.214 & 18.151 & 1.373 & 114.064 \\
\hline NITMET & 38.2 & 9.335 & 1.214 & 18.069 & 1.373 & 114.089 \\
\hline DMSO & 46.7 & 9.358 & 1.214 & 18.043 & 1.373 & 114.107 \\
\hline
\end{tabular}

Table 3.4 - Select solvent-dependent properties of C120. The dipole moment and amino bond angle increase with solvent dielectric constant while the dihedral angle of the amino group becomes more planar. These trends indicate an increase in charge transfer as solvent polarity increases. $\mathrm{CH}$ : Cyclohexane, CCl4: Carbon Tetrachloride, Tol: Toluene, $\mathrm{CHCl3}$ : Chloroform, THF: Tetrahydrofuran, Acetone: Acetone, NITMET: Nitromethane, DMSO: dimethyl sulfoxide.<smiles>Cc1cc(=O)oc2cc(N)ccc12</smiles><smiles>Cc1cc([O-])oc2cc(=[NH2+])ccc1-2</smiles>

Figure 3.7 - Resonance representation of C120. The left structure is thought to be predominant in nonpolar solvents while the right structure is thought to develop in polar solvents. 


\begin{tabular}{|c|c|c|c|c|c|c|c|}
\hline GAS & $\mathrm{CH}$ & CTCL & TOL & $\mathrm{CHCl} 3$ & ACETONE & NITMET & DMSO \\
\hline 692 & 692 & 692 & 692 & 691 & 691 & 691 & 691 \\
\hline 706 & 708 & 708 & 708 & 709 & 709 & 709 & 709 \\
\hline 725 & 725 & 726 & 725 & 725 & 727 & 728 & 728 \\
\hline 744 & 746 & 746 & 746 & 746 & 747 & 747 & 747 \\
\hline 802 & 807 & 808 & 808 & 810 & 811 & 811 & 811 \\
\hline 810 & 811 & 811 & 811 & 813 & 814 & 814 & 815 \\
\hline 849 & 847 & 847 & 847 & 845 & 843 & 843 & 843 \\
\hline 865 & 859 & 859 & 858 & 855 & 849 & 850 & 850 \\
\hline 866 & 871 & 872 & 872 & 875 & 878 & 879 & 879 \\
\hline 936 & 945 & 945 & 946 & 951 & 954 & 955 & 956 \\
\hline 987 & 986 & 986 & 986 & 986 & 986 & 986 & 986 \\
\hline 1023 & 1025 & 1024 & 1025 & 1025 & 1025 & 1024 & 1024 \\
\hline 1060 & 1058 & 1058 & 1058 & 1057 & 1056 & 1056 & 1056 \\
\hline 1071 & 1072 & 1071 & 1071 & 1072 & 1075 & 1076 & 1076 \\
\hline 1108 & 1109 & 1108 & 1108 & 1108 & 1108 & 1108 & 1108 \\
\hline 1146 & 1149 & 1149 & 1149 & 1150 & 1149 & 1149 & 1149 \\
\hline 1175 & 1173 & 1173 & 1173 & 1172 & 1171 & 1171 & 1171 \\
\hline 1227 & 1227 & 1227 & 1227 & 1226 & 1226 & 1226 & 1226 \\
\hline 1244 & 1245 & 1245 & 1245 & 1245 & 1239 & 1234 & 1234 \\
\hline 1282 & 1281 & 1281 & 1280 & 1279 & 1278 & 1279 & 1278 \\
\hline 1351 & 1351 & 1351 & 1351 & 1352 & 1353 & 1353 & 1353 \\
\hline 1374 & 1375 & 1375 & 1375 & 1375 & 1376 & 1376 & 1376 \\
\hline 1404 & 1400 & 1400 & 1399 & 1397 & 1399 & 1400 & 1400 \\
\hline 1423 & 1423 & 1423 & 1423 & 1423 & 1422 & 1422 & 1422 \\
\hline 1475 & 1472 & 1472 & 1471 & 1469 & 1469 & 1470 & 1470 \\
\hline 1484 & 1478 & 1478 & 1477 & 1473 & 1471 & 1471 & 1471 \\
\hline 1491 & 1481 & 1480 & 1479 & 1475 & 1481 & 1482 & 1482 \\
\hline 1544 & 1543 & 1543 & 1542 & 1542 & 1541 & 1542 & 1542 \\
\hline 1585 & 1579 & 1579 & 1578 & 1574 & 1571 & 1570 & 1570 \\
\hline 1653 & 1643 & 1641 & 1641 & 1633 & 1626 & 1626 & 1625 \\
\hline 1656 & 1651 & 1650 & 1650 & 1647 & 1644 & 1644 & 1644 \\
\hline 1670 & 1662 & 1661 & 1661 & 1657 & 1653 & 1652 & 1652 \\
\hline 1818 & 1785 & 1782 & 1780 & 1760 & 1744 & 1742 & 1741 \\
\hline
\end{tabular}

Table 3.5 - Calculated vibrational frequencies for $\mathrm{C} 120\left(\mathrm{in}^{-1}\right)$ using PCM. Frequencies correspond to those found experimentally in the FTIR and spontaneous Raman spectra. The carbonyl and C-N deformation modes are bolded for easy access. 


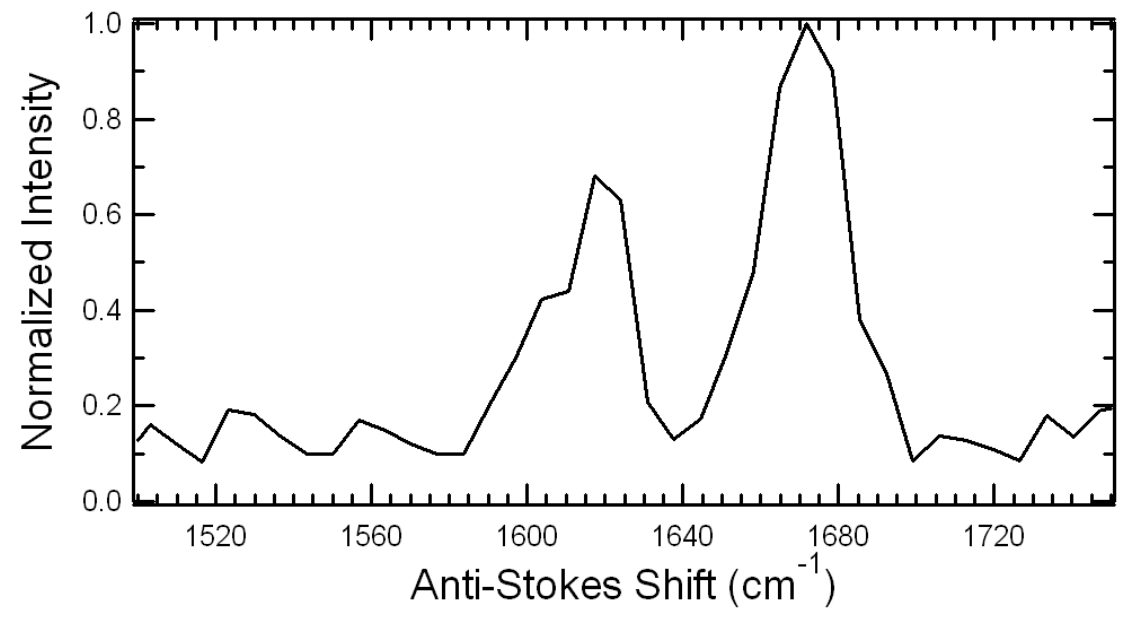

Figure 3.8 - fs/ps-CARS spectrum of C120 in DMSO in the carbonyl stretch region. There are three main peaks, the carbonyl at $1671 \mathrm{~cm}^{-1}$ and the two ring modes at $1606 \mathrm{~cm}^{-1}$ and $1621 \mathrm{~cm}^{-1}$. Spectrum is normalized to strongest feature present. 


\title{
CHAPTER 3: EXCITED STATE DYNAMICS OF LOW FREQUENCY MODES OF 4-DIMETHYLAMINO-4'NITROSTILBENE IN ACETONITRILE
}

\author{
Alex J. Blom, Benjamin D. Prince, Mikhail N. Slipchenko and Hans U. Stauffer
}

\subsection{Abstract}

The multiplexed coherent anti-Stokes Raman scattering probe fs/ps-CARS is used to monitor the evolution of low frequency vibrational modes in 4-dimethylamino-4'nitrostilbene. The ground state spectrum is assigned with assistance from quantum chemical calculations. Dynamics of excited state modes are observed for the first six picoseconds in acetonitrile and interpreted by comparing with transient electronic spectra. These results are evaluated with previous work to determine the relaxation pathway in highly polar solvents.

\subsection{Introduction}

Compounds capable of undergoing excited state charge transfer, often denoted as Donor-Acceptor (D-A) type molecules, have been studied for decades via various experimental techniques. ${ }^{1-19}$ The prototypical system for D-A systems is 4dimethylaminobenzonitrile (DMABN). This system has been studied by numerous groups in an attempt to understand how it is affected upon excitation. ${ }^{1-10}$ Rettig and coworkers have proposed the most widely accepted theory: following excitation, in polar solvents, DMABN undergoes a charge transfer from the dimethylamino group to the benzonitrile moiety, followed by a twisting dimethylamino group with respect to the 
plane of the molecule. Excitation induces the molecule to relax on its excited state potential energy surface, resulting in a complete decoupling of the D and A groups and effectively quenching fluorescence. ${ }^{10}$

D-A systems involving substituted stilbenes have recently gained interest for their potential use as molecular wires. One system in particular, 4-dimethylamino-4'nitrostilbene (DANS), shown in Figure 4.1, has been studied by several groups due to its interesting fluorescence properties. ${ }^{15-19}$ The quantum yield in DANS increases in going from nonpolar to slightly polar solvents and is accompanied by a red shift. The fluorescence is nearly reduced to zero in highly polar solvents while significantly shifting to the red. Rulliere et al have suggested an intriguing explanation for this behavior in analogy to that determined for the similar species 4-dimethylamino-4'-cyanostilbene. ${ }^{12-14}$ This interpretation involves solvent-dependent conformational changes of excited DANS. Upon excitation the molecule accesses the $\mathrm{E}^{*}$ (Franck-Condon) state. In slightly polar solvents the excited state is thought to twist about the 2 and 4 bonds $\left(\mathrm{A}_{2,4}{ }^{*}\right)$, as indicated in Figure 4.1. ${ }^{15.16}$ In highly polar solvents, on the other hand, a twist is thought to occur about the 5 bond $\left(\mathrm{A}_{5}{ }^{*}\right)$. In both cases it is likely that charge transfer accompanies structural changes, this is justified by the red-shift of fluorescence.

Vibrational studies of DANS have been limited to the fingerprint region. ${ }^{15,16,18}$ Work by Rulliere using coherent anti-Stokes Raman scattering suggested a dynamical change of modes associated with the nitro group in acetonitrile $(\mathrm{ACN})$ that they attributed to the formation of the $\mathrm{A}_{5}{ }^{*}$ twisted intramolecular charge transfer state (TICT). ${ }^{15,16}$ The resolution of their work was $\sim 1$ ps and any changes occurring on faster timescales could not be observed. Work in our group presented recently using the multiplexed fs/ps-CARS 
( 100 fs time resolution) probe of excited state dynamics has indicated that in highly polar solvents the transition from the Franck-Condon (FC) state to any subsequent states occurs on very fast timescales. ${ }^{20}$

To this point there have been no studies conducted to observe the low frequency vibrations in DANS in the excited state. Modes in the $300-800 \mathrm{~cm}^{-1}$ regime typically involve bending motions of substituents, out of plane motions of the entire system, and torsional modes of the backbone. To that end we have used a multiplexed Raman probe termed fs/ps-CARS to measure the low frequency modes of DANS in ACN.

Understanding how the low frequency vibrations of this system are affected upon excitation can help determine the relaxation pathways it undergoes in the excited state.

The fs/ps-CARS probe allows for time resolution on the order of $100 \mathrm{fs}$ and frequency resolution of better than $12 \mathrm{~cm}^{-1} \cdot{ }^{21}$ Use of a femtosecond, broadband $\omega_{1}$ and $\omega_{2}$ pulse followed by a narrowband, picosecond $\omega_{3}$ pulse allows for multiplexed detection of Raman-active vibrations. Previous work in our group has confirmed the validity of the probe's resolution. Transient excited states can be monitored using the fs/ps-CARS technique through use of a broadband femtosecond pump pulse, typically $25000 \mathrm{~cm}^{-1}$ in energy $(400 \mathrm{~nm})$.

This study is organized as follows. Ground state modes of DANS in ACN are measured using the fs/ps-CARS probe technique and are assigned with assistance from quantum chemical calculations. Excited state spectra are obtained using the pump-probe technique with a $400 \mathrm{~nm}$ pump pulse followed by the fs/ps-CARS probe. The spectrum of the excited state is measured for the first six picoseconds. Transient electronic spectra using $400 \mathrm{~nm}$ pump and various probe wavelengths $(511 \mathrm{~nm}, 550 \mathrm{~nm}$, or $800 \mathrm{~nm})$ are 
measured to compare the excited state electronic evolution to excited state vibrational evolution. Results are compared to previous work and a portrait is drawn as to the pathway for relaxation in the excited state.

\subsection{Methods}

\subsubsection{Experimental Setup}

The fs/ps-CARS setup has been described in detail in an earlier paper. ${ }^{21}$ In short, a Ti:Sapphire laser system yields $800 \mathrm{~nm}(\sim 50 \mathrm{fs})$ pulses repeating at $1 \mathrm{kHz}$ with typical powers of $2 \mathrm{~mJ} /$ pulse. The output beam is separated into three distinct portions. The first is sent into an optical parametric amplifier (OPA) that produces ultrafast broadband pulses which, after combination with the fundamental yields pulses in the visible region $\left(\omega_{1}\right.$ and $\left.\omega_{2}\right)$. For these experiments the OPA was tuned so that the energy difference between the two visible pulses was $\omega_{1}-\omega_{2}=358 \mathrm{~cm}^{-1}\left(\omega_{1}=19084 \mathrm{~cm}^{-1}[524 \mathrm{~nm}]\right.$ and $\left.\omega_{2}=18726 \mathrm{~cm}^{-1}[534 \mathrm{~nm}]\right)$ to monitor low frequency vibrations. A grating-stretcher type pulse shaping apparatus is used to convert a second part of the fundamental into picosecond duration pulses. ${ }^{22}$ This yields narrowband pulses $\left(\sim 8 \mathrm{~cm}^{-1}\right)$ whose width is controlled through use of an adjustable slit $\left(\omega_{3}\right)$. The three beams, $\omega_{1}, \omega_{2}, \omega_{3}$ make up the fs/ps-CARS probe. For excited state experiments the remaining fundamental is used to produce ultrafast pulses at $400 \mathrm{~nm}\left(\omega_{\mathrm{ex}}\right)$. This beam is produced through frequency doubling of the fundamental using a BBO doubling crystal. All beam polarizations are set to parallel.

The pulses are directed into the sample using a folded BOXCARS geometry. This specific beam pattern allows the signal beam to be spatially separated from the input 
beams and is then spectrally dispersed in a spectrometer made up of a monochromator (Jarrell Ash, $f=300 \mathrm{~mm}$ ) outfitted with a 1200 line/mm grating and a Princeton Instruments 1100 element CCD array detector. This setup allows for resolution of up to $1.2 \mathrm{~cm}^{-1} /$ pixel. The spectrometer is calibrated using a chloroform standard. Frequencies observed from the standard are then compared with tabulated values in the desired frequency range $\left(350-800 \mathrm{~cm}^{-1}\right)$. Excited state spectra were acquired by preparing an electronically excited state using $\omega_{\mathrm{ex}}$ followed by the fs/ps-CARS probe. The $400 \mathrm{~nm}$ excitation pulse is directed into the sample in the center of the folded BOXCARS geometry. A computer-controlled delay stage is used to adjust the delay between $\omega_{\mathrm{ex}}$ and the fs/ps-CARS probe to measure excited state dynamics. The overlap of $\omega_{\mathrm{ex}}$ with the remaining pulses (time zero) is defined using the optical Kerr effect in glass.

Transient absorption/emission spectra were obtained with $400 \mathrm{~nm}$ excitation followed by a probe pulse $(511 \mathrm{~nm}, 550 \mathrm{~nm}$, or $800 \mathrm{~nm})$. The OPA was tuned from the values listed for fs/ps-CARS experiments so three regions of the electronic spectrum could be monitored. The $400 \mathrm{~nm}$ (pump) pulse was directed into the sample and overlapped with the probe pulse. After the sample the beams are spatially separated and the probe pulse is directed into a photodiode (Thorlabs model DET210) with the intensity monitored using a lock-in amplifier. Again, the delay of the pump pulse relative to the probe pulse was controlled via a computer-driven delay stage in the pump beam path.

\subsubsection{Materials}

Trans-4-dimethylamino-4'-nitrostilbene (DANS) was obtained from Aldrich (97\%). HPLC grade acetonitrile (ACN) was obtained from Fisher for use as a solvent and 
as a Raman standard. Toluene (certified ACS) was also obtained from Fisher. For these experiments DANS was solvated in ACN to typical concentrations of $1 \mathrm{mM}$. The sample is placed in the $220 \mu \mathrm{m}$ space between two fused silica windows in a rotating cell. The cell connected to a motor and spun to ensure complete sample refreshment between laser shots.

\subsubsection{Computational Methods}

Density Functional Theory (DFT) has been used with the B3LYP functional and a 6-31G(d,p) basis set to determine harmonic vibrations of DANS. All calculations were conducted using the GAMESS software. ${ }^{23}$ The DFT level of theory, especially with the B3LYP functional is known to give reliable geometries and vibrational frequencies. Additionally, it gives a good combination of accurate results and low computational time. Geometry optimizations were first conducted and a Hessian run was then performed to confirm this structure as a minimum. This second calculation also yields the molecular frequencies. Raman and infrared intensities of the frequencies were found using the GAMESS default field strengths. ${ }^{23}$

\subsection{Results}

\subsubsection{Ground State Spectrum of DANS}

The ground state spectrum of DANS in ACN obtained using the low frequency fs/ps-CARS probe is shown in Figure 4.2. The most intense feature in this spectrum is the $380 \mathrm{~cm}^{-1}$ mode of the solvent. There are three ground state vibrations clearly visible in 
this spectrum at $526 \mathrm{~cm}^{-1}, 633 \mathrm{~cm}^{-1}$ and at $760 \mathrm{~cm}^{-1}$. These frequencies were compared to those calculated using GAMESS and are assigned in Table 4.1.

It is typical to scale harmonic frequencies calculated using DFT but in this case the values shown are not scaled. At the DFT B3LYP level using a 6-31G(d,p) basis set the tabulated scale factor is $0.961 \pm 0.045 .{ }^{24}$ Hence the scaling for test set molecules ranges from .916 to 1.006 . Correlating the experimental values to the theoretical ones was straightforward without the scaling factor.

The vibrations observed in the low frequency spectrum of DANS in ACN are shown in Figure 4.3. At $526 \mathrm{~cm}^{-1}$ the major component of the mode is the in-plane bending of the nitro group. There is also a small amount of twisting of the phenyl ring the nitro group is attached to. The $633 \mathrm{~cm}^{-1}$ mode is assigned to the $6 \mathrm{~b}$ vibration of both rings. This assignment is in agreement with the previous work of Varsanyi. ${ }^{24}$ The $6 \mathrm{~b}$ mode is fairly easy to distinguish in Figure 4.3 and involves the ring carbons bound to hydrogens. The final mode at $760 \mathrm{~cm}^{-1}$ is assigned as the 5 stretch of both rings. This vibration is out of the plane of the phenyl rings and involves the entire molecule.

\subsubsection{Excited State of DANS}

A graph presenting the excited state dynamics in the first six picoseconds of DANS in ACN is shown in Figure 4.4. The entire excited state range is shown. The full data set is split into two separate frequency regions to ease interpretation. At $0.0 \mathrm{ps} \omega_{\mathrm{ex}}$ initiates the excited state of DANS and the vibrations associated with this state follow. There are several modes present in the $S_{1}$ state, including $330 \mathrm{~cm}^{-1}$, the solvent peak at $380 \mathrm{~cm}^{-1}, 450 \mathrm{~cm}^{-1}, 640 \mathrm{~cm}^{-1}, 710 \mathrm{~cm}^{-1}$, and $760 \mathrm{~cm}^{-1}$. Additionally, there are three 
indistinct peaks in the $490-540 \mathrm{~cm}^{-1}$ region. Peaks were fit using Lorentzian functions to determine how the modes change as the excited state evolves. The fits yielded frequencies, intensities, and FWHM values.

The peak located at $330 \mathrm{~cm}^{-1}$ appears shortly after excitation and quickly shifts to a higher frequency before decaying to zero in the first 6 ps. The dynamics of this peak are outlined in Figure 4.5. In 4.5C it is seen that the peak appears after excitation and rises to a maximum within the duration of the pulse ( $100 \mathrm{fs})$. The intensity then begins to decrease until the peak completely disappears by 6 ps. At the same time the frequency increases on a timescale convoluted with our instrumental response and then continues to steadily move upward while the intensity of the feature decays to zero. The FWHM of this peak begins very broad at over $20 \mathrm{~cm}^{-1}$ and decreases to less than $15 \mathrm{~cm}^{-1}$ by the end of the experiment.

Located at $450 \mathrm{~cm}^{-1}$ is a peak (Figure 4.6) that appears only after photo-excitation and remains for the duration of the experiment. The frequency increases quickly while the excitation pulse is present, after which it gradually begins to decrease by a small amount $\left(\sim .5 \mathrm{~cm}^{-1}\right)$. The intensity of this mode also increases during the excitation pulse and slowly decreases for the remaining 6 ps, but never fully disappears. The width of the mode is constant at $10 \mathrm{~cm}^{-1}$.

Figure 4.7 outlines the evolution of the $640 \mathrm{~cm}^{-1}$ feature. This mode represents how the $6 \mathrm{~b}$ stretch of both rings changes upon excitation. The peak begins lower in frequency than its ground state counterpart, starting at $628 \mathrm{~cm}^{-1}$ and quickly shifting to higher frequency by $8 \mathrm{~cm}^{-1}$ before gradually increasing to $638 \mathrm{~cm}^{-1}$. The width and intensity of the peak also increase rapidly initially before gradually decreasing. The width 
settles near $10 \mathrm{~cm}^{-1}$ but the intensity continues to decrease through the end of the experiment.

Figure 4.8 details the dynamics of the two modes higher than $700 \mathrm{~cm}^{-1}$. The frequency of the $710 \mathrm{~cm}^{-1}$ mode increases rapidly initially and then slowly decreases. This peak has a very low intensity and fits were noisy. It is seen that the intensity has very gradual intensity change, increasing slowly upon excitation and then, once reaching a maximum, slowly decreasing. This mode does not, however, completely decay.

The $760 \mathrm{~cm}^{-1}$ peak outlined in Figure 4.8 describes the change of the ground state out of plane deformation mode of the same frequency. Its frequency decreases rapidly upon excitation before increasing back to the ground state value within 166 fs. The intensity begins at a maximum before decaying to a fairly constant value by the end of the experiment.

In order to further understand the dynamics of the excited state, transient electronic spectra at several wavelengths were acquired. The change in the pump pulse was measured as the delay of the $400 \mathrm{~nm}$ timing was changed. Traces for these experiments are shown in Figure 4.9 The decays of the transient spectra were fit to either one or two exponentials and the results are shown in Table 4.2. The $511 \mathrm{~nm}$ trace shows a mono-exponential decay with a time constant of $18 \mathrm{ps}$. The $550 \mathrm{~nm}$ trace has a biexponential decay fit to $\tau_{1}=391$ fs and $\tau_{1}=4$ ps. The transient emission trace at $800 \mathrm{~nm}$ has decay constants of $\tau_{1}=681 \mathrm{fs}$ and $\tau_{1}=12 \mathrm{ps}$, respectively. In all cases the transient spectra display a finite amount of intensity at $6 \mathrm{ps}$, the end of the fs/ps-CARS data.

These traces all show an immediate increase in magnitude upon excitation followed by a subsequent decay. If the FC state is accessed and evolves into the TICT 
state, it would be reasonable to expect the time-resolved electronic spectrum to begin near the steady-state fluorescence wavelength in nonpolar solvents $(500 \mathrm{~nm})$. The spectrum should then exhibit a red shift as the molecule develops into the TICT state. These three traces, however, indicate that the feature at $511 \mathrm{~nm}$ is long-lived while those at $550 \mathrm{~nm}$ and $800 \mathrm{~nm}$ quickly decay.

\subsection{Discussion}

The structure of DANS in the excited state is markedly different than in the ground state. The ground state modes observed at 632.8 and $760.5 \mathrm{~cm}^{-1}$ undergo significant changes upon excitation. The $632.8 \mathrm{~cm}^{-1}$ mode shows that the $6 \mathrm{~b}$ motions of the rings are continuously changing in the excited state. As the frequency shift occurs the peak broadens and since the width of the peak is inversely related to its dephasing time, it suggests this vibration becomes less stable initially. After 1 ps the width of this peak begins to decrease, indicating a stabilization of the system. The intensity of this peak does not return to zero in the first 6 ps but Figure 4.4B and 4.8 show that the intensity is decreasing when the experiment ends.

The $760.5 \mathrm{~cm}^{-1}$ mode, which is the out of plane deformation of DANS in the ground state, also changes upon excitation. Appearing immediately after excitation, this peak decreases in intensity and then increases to a steady value, remaining relatively intense for the duration of the experiment. A change in this mode requires a change in conformation of the entire molecule. The final frequency reached indicates that, upon stabilizing, the overall frequency of this vibration is the same as in the ground state. This does not mean the structures of the two species are the same, but that the molecule is 
changing in such a way that this vibration yields the same frequency as the ground state equivalent.

The peaks at $330 \mathrm{~cm}^{-1}, 450 \mathrm{~cm}^{-1}$ and $710 \mathrm{~cm}^{-1}$ are unique to the excited state configuration of DANS. In the 330 mode frequency quickly (instrument limited) jumps upward $25 \mathrm{~cm}^{-1}$ to $355 \mathrm{~cm}^{-1}$ then continues to increase until the peak disappears near $6 \mathrm{ps,}$ merging into the ACN mode at $380 \mathrm{~cm}^{-1}$. The $450 \mathrm{~cm}^{-1}$ mode exhibits the same initial frequency increase, but decreases in frequency after $1 \mathrm{ps}$. The width of the two peaks is also interesting. The 330 mode starts around $25 \mathrm{~cm}^{-1} \mathrm{FWHM}$, and exhibits an instrumentlimited decrease, followed by a gradual decrease until the end of the experiment. The $450 \mathrm{~cm}^{-1}$ mode, on the other hand, maintains a fairly constant width throughout the experiment. The $710 \mathrm{~cm}^{-1}$ peak exhibits a frequency profile similar to the $450 \mathrm{~cm}^{-1}$ mode. The width of this peak is less stable, however, indicating a changing vibration.

Excited state modes are difficult to assign without isotopic substitution unless they correspond to a ground state frequency. With this in mind the (very) tentative assignments for the pure excited state modes have been made. The feature near $330 \mathrm{~cm}^{-1}$ is assigned to the stretch of the phenyl-nitro group, with the entire nitro group moving in unison. The $450 \mathrm{~cm}^{-1}$ feature is considered to be a motion of the dimethylamino substituent, likely an in-plane bending motion. Finally, the $710 \mathrm{~cm}^{-1}$ mode is likely an out of plane deformation mode. Again, assignments of these modes are tentative and further work in other solvents and using isotopic substitution or similar molecules would help discern the identity of these modes.

A model proposed by Rulliere and coworkers suggests that there are multiple excited state configurations in DANS. ${ }^{15,16}$ In highly polar solvents such as ACN the 
molecule is thought to initially access the Franck-Condon state before evolving to a twisted configuration termed the $\mathrm{A}_{5}{ }^{*}$ state. This state involves twisting of the nitro group. Using transient absorption experiments they attributed a bi-exponential decay of $1 \mathrm{ps}$ and $13 \mathrm{ps}$ to the nonradiative relaxation of the $\mathrm{A}_{5}{ }^{*}$ state. They also attributed a stimulated emission band at $750 \mathrm{~nm}$ with an overall lifetime less than $2 \mathrm{ps}$ to the decay of the Franck-Condon state. Assignment of this emission to the FC state is inconsistent with steady-state fluorescence, however, which should occur at 500-700 nm.

The transient electronic spectra shown in Figure 4.9 indicate that the state initially accessed upon excitation fluoresces at $800 \mathrm{~nm}$ for several picoseconds before decaying away. A trace at $550 \mathrm{~nm}$ indicates a short-lived transient absorption that is gone in less than 5 ps while the $511 \mathrm{~nm}$ trace shows that the first excited configuration remains for quite some time. The $511 \mathrm{~nm}$ trace shows an exponential decay of 23 ps with intensity remaining for over $50 \mathrm{ps}$. These electronic spectra indicate that immediately following excitation a state is accessed that can simultaneously display transient absorption at 511 $\mathrm{nm}$ and $550 \mathrm{~nm}$ while emitting at $800 \mathrm{~nm}$. It is unlikely that these dynamics belong to the FC state since the steady state fluorescence of this state has no intensity at $800 \mathrm{~nm}$.

The long lifetime of the $511 \mathrm{~nm}$ transient absorption indicates the molecule enters into a state that is fairly stable. The $550 \mathrm{~nm}$ trace suggests the molecule is relaxing on the potential energy surface in the first several picoseconds. The first time component of 391 fs associated with this trace is likely due to solvent reorganization around the excited species. The $800 \mathrm{~nm}$ emission present initially suggests the state accessed has some overlap with the ground state. Since this feature appears upon excitation, the molecule is likely in a region of the potential energy surface where the excited state configuration is 
slightly relaxed while the corresponding ground state configuration is high in energy. This scenario results in the $800 \mathrm{~nm}\left(12500 \mathrm{~cm}^{-1}\right)$ fluorescence. SAM1 calculations of Ernsting show that a twist of the nitro group results in the destabilization of the ground state and supports this theory. ${ }^{18}$

The peaks observed in the excited state all appear immediately after excitation. As in the transient electronic spectra, the lack of a delay between excitation and the evolution of the peaks implies that if the FC state and any subsequent TICT state exhibit a precursor-successor relationship, it is not being observed here. A population transfer from the FC to TICT state would likely be accompanied by the decay of vibrational modes associated with the FC and the growth of modes in the TICT.

It could be reasoned that the pulse-limited activity of the vibrations in this system are an indication of the FC state evolving into some successive conformation, especially since the three observed ground state modes all appear in the excited state. In this case it might be argued that the FC state is present at very early times and has a very short $(<100$ fs) lifetime. Additionally, if the vibrations of the FC state were weak or highly nonresonant, they would be difficult to observe. The structure of the FC state should be very similar to the ground state, however, and the presence of three completely new peaks, in addition to the radical changes seen in the ground state modes indicate that the FC state not being observed.

It is likely that the molecule is excited to and evolves out of the FC state within the duration of the pulse. This scenario is supported by the instantaneous growth of three distinct excited state peaks, distinct changes observed in the ground state modes upon excitation, and transient electronic spectra. Additionally, the lack of features attributable 
to the FC state support this thought. The nitro bending motion $\left(\sim 500 \mathrm{~cm}^{-1}\right)$ shifts downward and disappears within 1 ps. The out of plane deformation of the molecule radically changes, alternately decreasing and increasing in frequency. The $6 \mathrm{~b}$ motions of the rings shift upward to a steady frequency and remain there to the end of the experiment. These factors, considered in conjunction with the presence of three new peaks indicate that conformation being observed is very different in geometry than the ground state and is likely not the FC state.

The state assigned as the $\mathrm{A}_{5}{ }^{*}$ configuration of the excited state is likely accessed upon excitation. This state results from twisting of the nitro group relative to the plane of the stilbene moiety and a simultaneous transfer of charge from the dimethylamino donor group to the nitro acceptor. Observed changes in the ground state ring motions, especially the out of plane deformation seem to support this assignment. Changes of the $330 \mathrm{~cm}^{-1}$ phenyl-nitro stretch also confirm this since this mode undergoes an increase in frequency, indicating a dynamic change of this bond.

\subsection{Conclusions}

The vibrational spectrum of DANS in ACN in the low frequency $\left(300-800 \mathrm{~cm}^{-1}\right)$ regime has been observed for the ground state and the first six picoseconds of the first excited singlet state using the fs/ps-CARS method. It is seen that the three ground state vibrations change significantly in the excited state and, in addition, three new peaks are

observed. It is thought that these peaks are representative of a TICT state, likely the $\mathrm{A}_{5}{ }^{*}$ state as assigned by Rulliere and coworkers. The FC state was not observed, however, in this study. Transient electronic spectra at several wavelengths following $400 \mathrm{~nm}$ 
excitation support the lack of the FC state being observed. It is likely that the FC state is accessed very quickly following excitation and the molecule evolves out of this state within the duration of the pulse. Alterations of the ground state modes, combined with dynamics of pure excited state modes indicate the nitro group is undergoing a radical change in this state.

\subsection{References}

1. A. Demeter and K. A. Zachariasse, Chem. Phys. Lett., 308, 699 (2003)

2. J. Dreyer and A. Kummrow, J. Am. Chem. Soc., 122, 2577 (2000)

3. S. I. Druzhinin, N. P. Ernsting, S. A. Kovalenko, L. W. Lustres, T. A. Senyushkina, and K. A. Zachariasse, J. Phys. Chem. A, 110, 2955 (2000)

4. W. M. Kwok, C. Ma, D. Phillips, P. Matousek, A. W. Parker and M. Towrie, J. Phys. Chem. A, 104, 4188, (2000).

5. H. Okamoto, J. Phys. Chem. A, 104, 4182 (2000)

6. H. Okamoto, H. Inishi, Y. Nakamura, S. Kohtani, and R. Nakagaki, J. Phys. Chem. A, 105, 4182 (2001)

7. K. A. Zachariasse, M. Grobys and E. Tauer, Chem. Phys. Lett., 274, 372 (1997)

8. K. A. Zachariasse, Chem. Phys. Lett., 320, 8 (2000)

9. K. Rotkiewicz, K. H. Grellman and Z. R. Grabowski, Chem. Phys. Lett., 19, 315 (1973)

10. Z. R. Grabowski, K. Rotkiewicz and W. Rettig, Chem. Rev., 103, 3899 (2003)

11. H. Görner and H. J. Kuhn, Adv. Photochem., 19, 1 (1995)

12. Y. V. Il'chev, W. Kühnle and K. A. Zachariasse, Chem. Phys., 211, 441 (1996)

13. W. Rettig and W. Majenz, Chem. Phys. Lett., 154, 335 (1989) 
14. R. Lapouyade, A. Kuhn, J. F. Letard and W. Rettig, Chem. Phys. Lett, 208, 48 (1993)

15. J. Oberlé, E. Abraham, G. Jonusauskas and C. Rulliére, J. Ramam. Spectrosc, 31, 311 (2000)

16. J. Oberlé, G. Jonusauskas, E. Abraham, R. Lapouyade, and C. Rulliére, B. Chem. Soc. Jpn., 75, 1041 (2002)

17. H. Gruen and H. Görner, J. Phys. Chem., 93, 7144 (1989)

18. V. M. Farztdinov and N. P. Ernsting, Chem. Phys, 277, 257 (2002)

19. T. Vijayakumar, I. Hubert Joe, C. P. Reghunadhan Nair, V. S. Jayakumar, Chem. Phys., 343, 83 (2008).

20. B. M. Prince, B. D. Prince, A. Chakraborty, M. N. Slipchenko, and H. U. Stauffer, J. Raman. Spectrosc., manuscript in preparation

21. B. D. Prince, A. Chakraborty, B. M. Prince, and H. U. Stauffer, J. Chem. Phys, 125, 044502 (2006)

22. A. M. Weiner, Rev. Sci. Instrum., 71, 1929 (2000)

23. M. W. Schmidt, K. K. Baldridge, J. A. Boatz, S. T. Elbert, M. S. Gordon, J. H. Jensen, S. Koseki, N. Matsunaga, K. A. Nguyen, S. J. Su, T. L Windus, M. Dupuis, J. A. Montgomery, J. Comp Chem 14, 1347 (1993).

24. Computational Chemistry Comparison Benchmark Database; URL; http://cccbdb.nist.gov/, last accessed 6-21-09.

25. G. Varsanyi, Assignments for vibrational spectra of seven hundred benzene derivatives; Wiley, 1974 


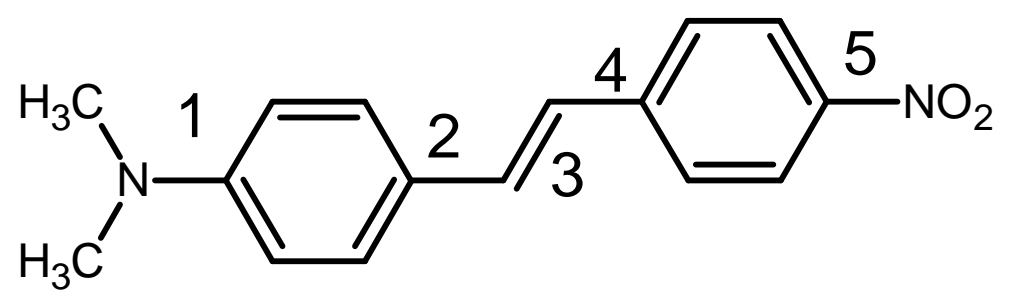

Figure 4.1 - Molecular Structure of 4-dimethylamino-4'-nitrostilbene (DANS). The numbering scheme is typical of para disubstituted stilbene molecules.

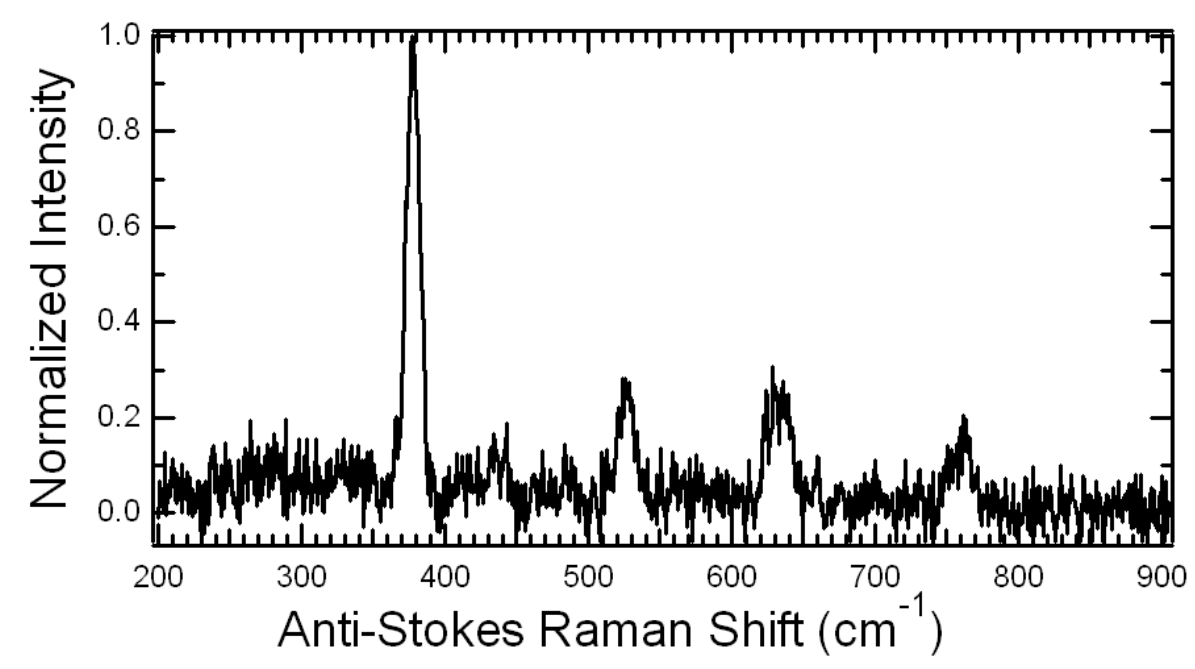

Figure 4.2 - Low frequency fs/ps-CARS spectrum of DANS in ACN.

\begin{tabular}{|l|l|l|l|}
\hline $\mathbf{v}_{\text {CARS }}\left(\mathbf{c m}^{-1}\right)$ & v $_{\text {CALC }}\left(\mathbf{c m}^{-1}\right)$ & $\begin{array}{l}\text { Wilson Notation } \\
\text { assignment }\end{array}$ & Description \\
\hline 526.4 & 549 & $\beta_{\text {as }} \mathrm{NO}_{2}$ & $\begin{array}{l}\text { Nitro in plane antisymmetric } \\
\text { bend }\end{array}$ \\
\hline 632.8 & 658 & $6 \mathrm{~b}$ (Both Rings) & $\begin{array}{l}\text { Ph CC Str + Ph C-H Str Ph- } \\
\text { NO } \text { IP deformation }\end{array}$ \\
\hline 760.5 & 760 & 5 (Both Rings) & $\begin{array}{l}\text { OOP Molecular } \\
\text { Deformation }\end{array}$ \\
\hline
\end{tabular}

Table 4.1 - Assignments of low frequency ground state vibrations of DANS in ACN. 

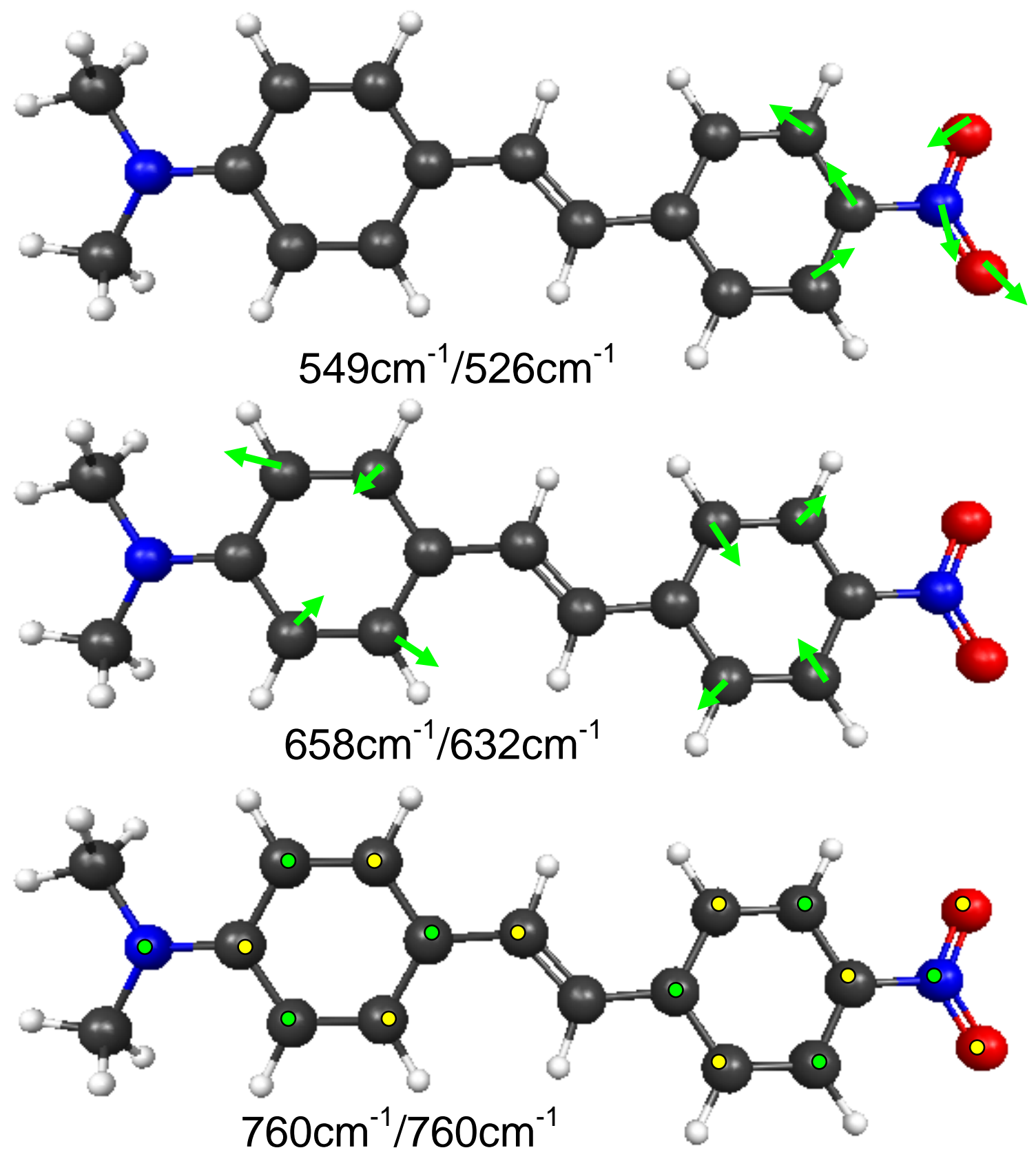

Figure 4.3 - Vibrations of DANS observed experimentally [formatted (calc/exp)]. Atomic displacements indicated with arrows. For the $760 \mathrm{~cm}^{-1}$ vibration the out of plane motions are indicated with colored circles. Green circles indicate motion upward, out of the plane of the paper while yellow circles indicate downward motion into the plane of the paper. 

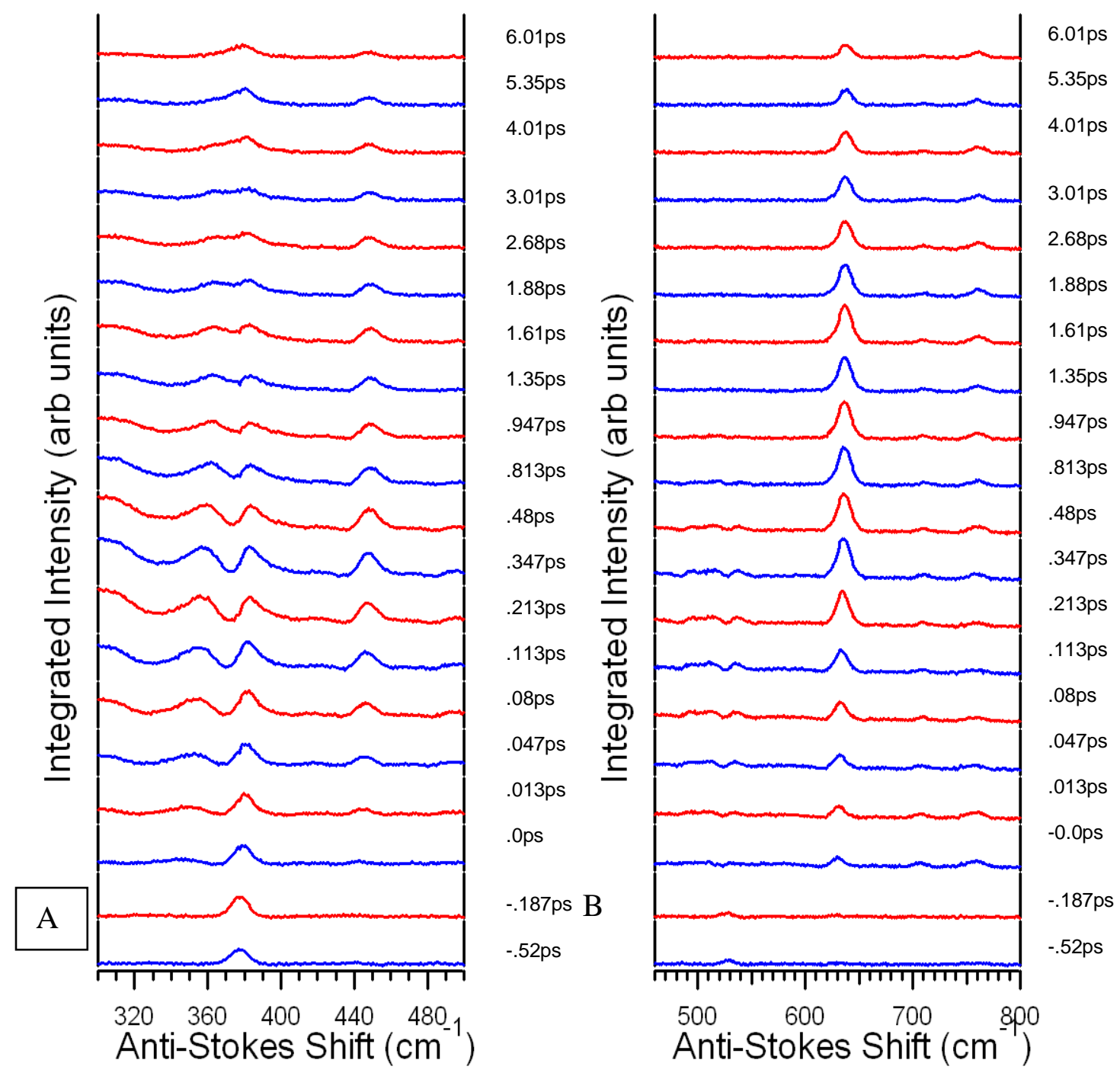

Figure 4.4 - A) Excited state spectra in $300-500 \mathrm{~cm}^{-1}$ region. Two peaks are present, the first near $350 \mathrm{~cm}^{-1}$ and the other at $450 \mathrm{~cm}^{-1}$. As mentioned previously, the $380 \mathrm{~cm}^{-1}$ peak is due to the solvent. B) Excited state spectra in the $480-800 \mathrm{~cm}^{-1}$ region. Major peaks are located at 640,710 , and $760 \mathrm{~cm}^{-1}$. There are several weak, hard to define peaks in the $540 \mathrm{~cm}^{-1}$ region. 

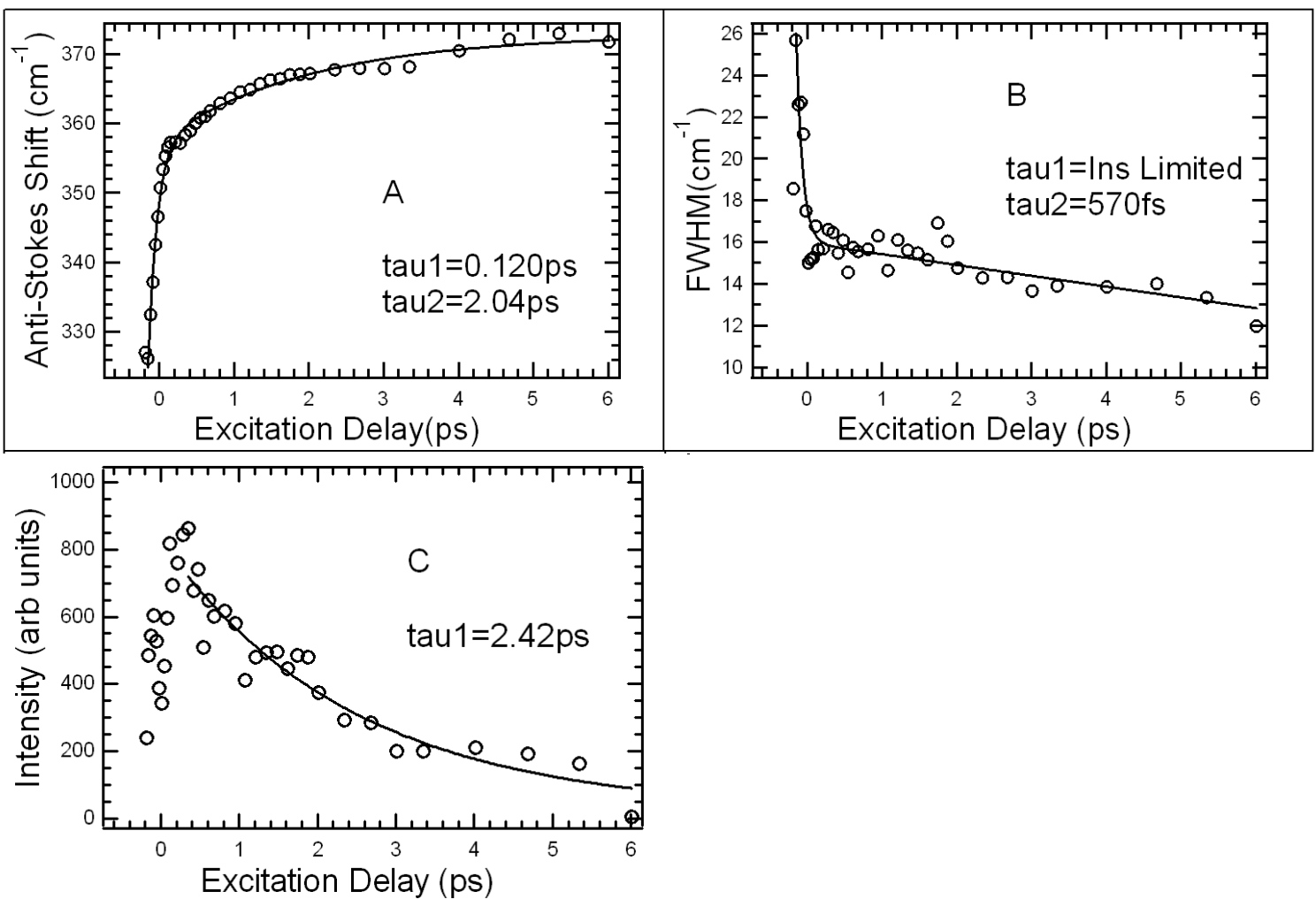

Figure 4.5 - Behavior of the $330 \mathrm{~cm}^{-1}$ excited state peak. A) The frequency undergoes a bi-exponential increase in value. B) After an initial instrument-limited response, the full width half max of the peak steadily narrows. C) The intensity exhibits an instrument-limited rise followed by a 2.42 ps decay until reaching zero at $6 \mathrm{ps}$.

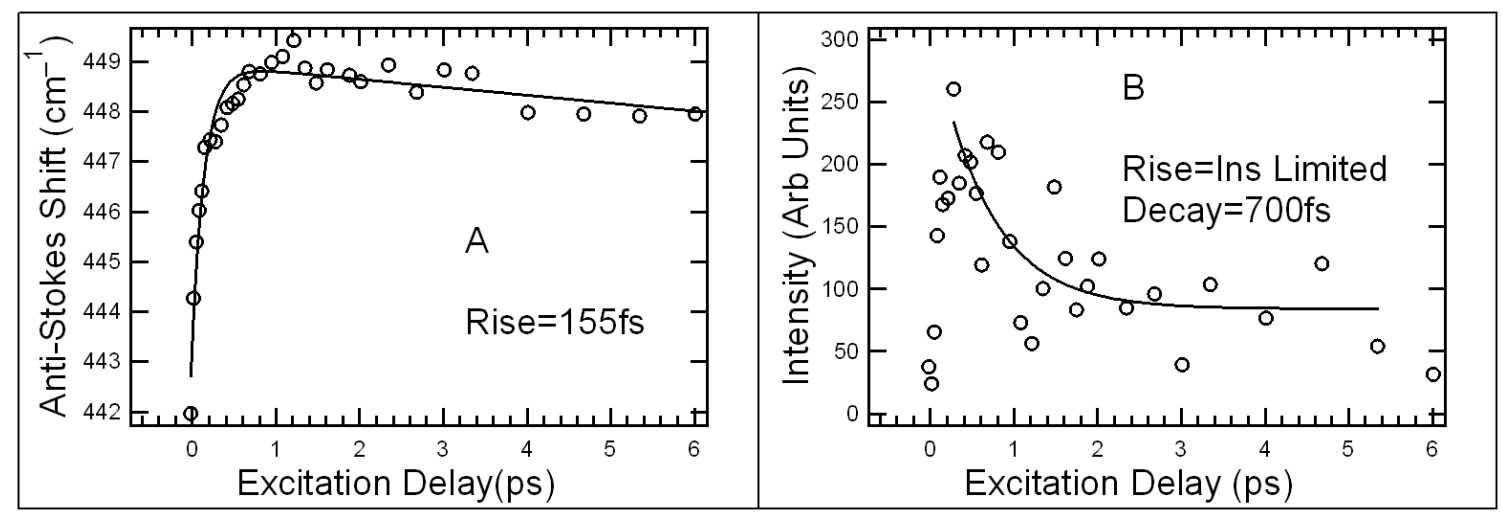

Figure 4.6 - Behavior of peak near $440 \mathrm{~cm}^{-1}$. A) Upon excitation this peak appears and increases in frequency with a $155 \mathrm{fs}$ timescale. It then begins to decrease in frequency until the peak disappears. B) The intensity of this peak shows an instrument-limited increase after excitation followed by a $700 \mathrm{fs}$ decay away. The width of this peak is constant (within experimental error) at $10 \mathrm{~cm}^{-1} \mathrm{FWHM}$. 


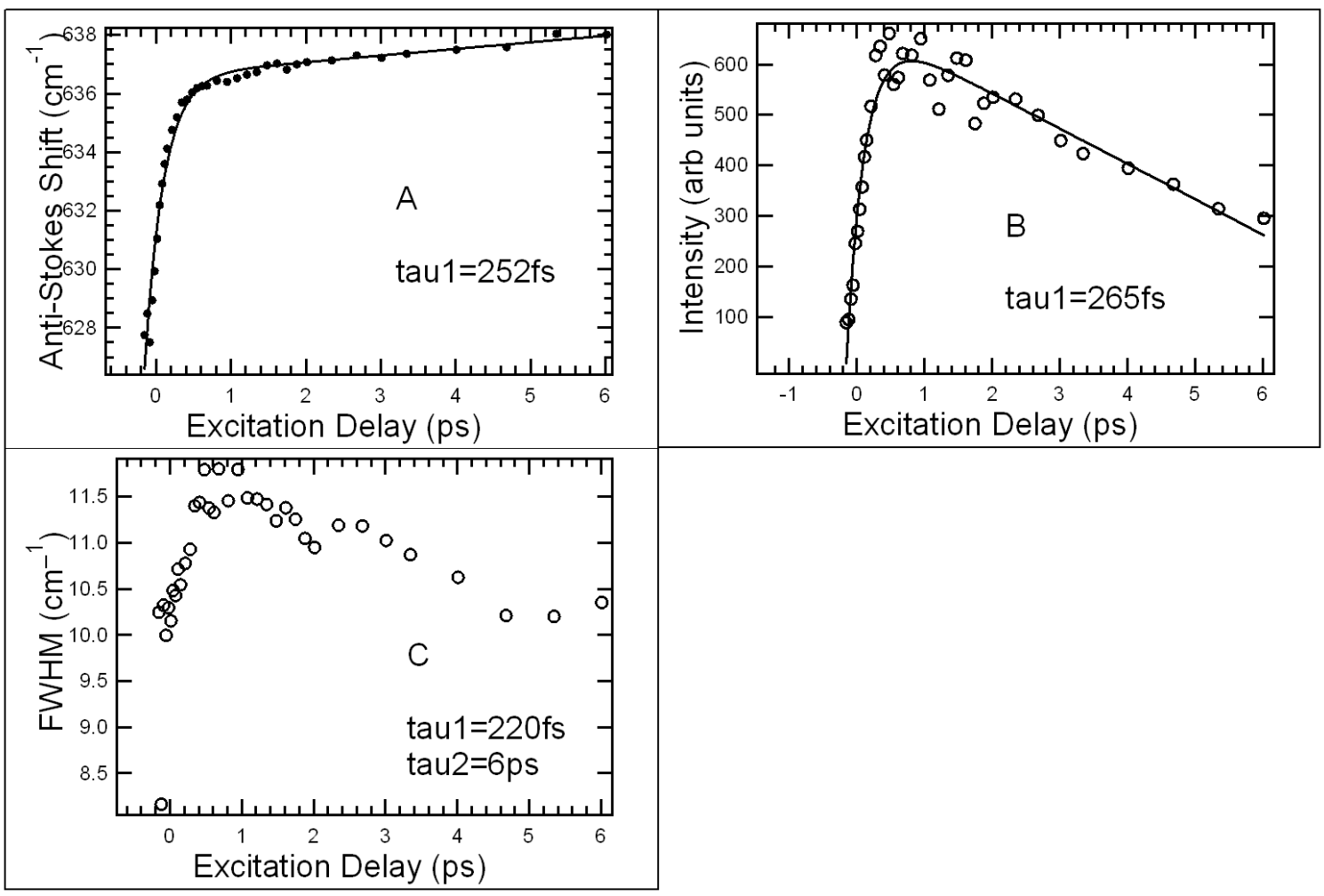

Figure 4.7 - Dynamics of $640 \mathrm{~cm}^{-1}$ Excited State peak. A) There is a $252 \mathrm{fs}$ upshift in frequency followed by a gradual continuing upshift which could not be fit reliably. B) The intensity of the peak increases rapidly (265fs) upon excitation and then begins to decrease but the peak remains at the end of the experiment. C) The width of the peak behaves similarly to the intensity. 


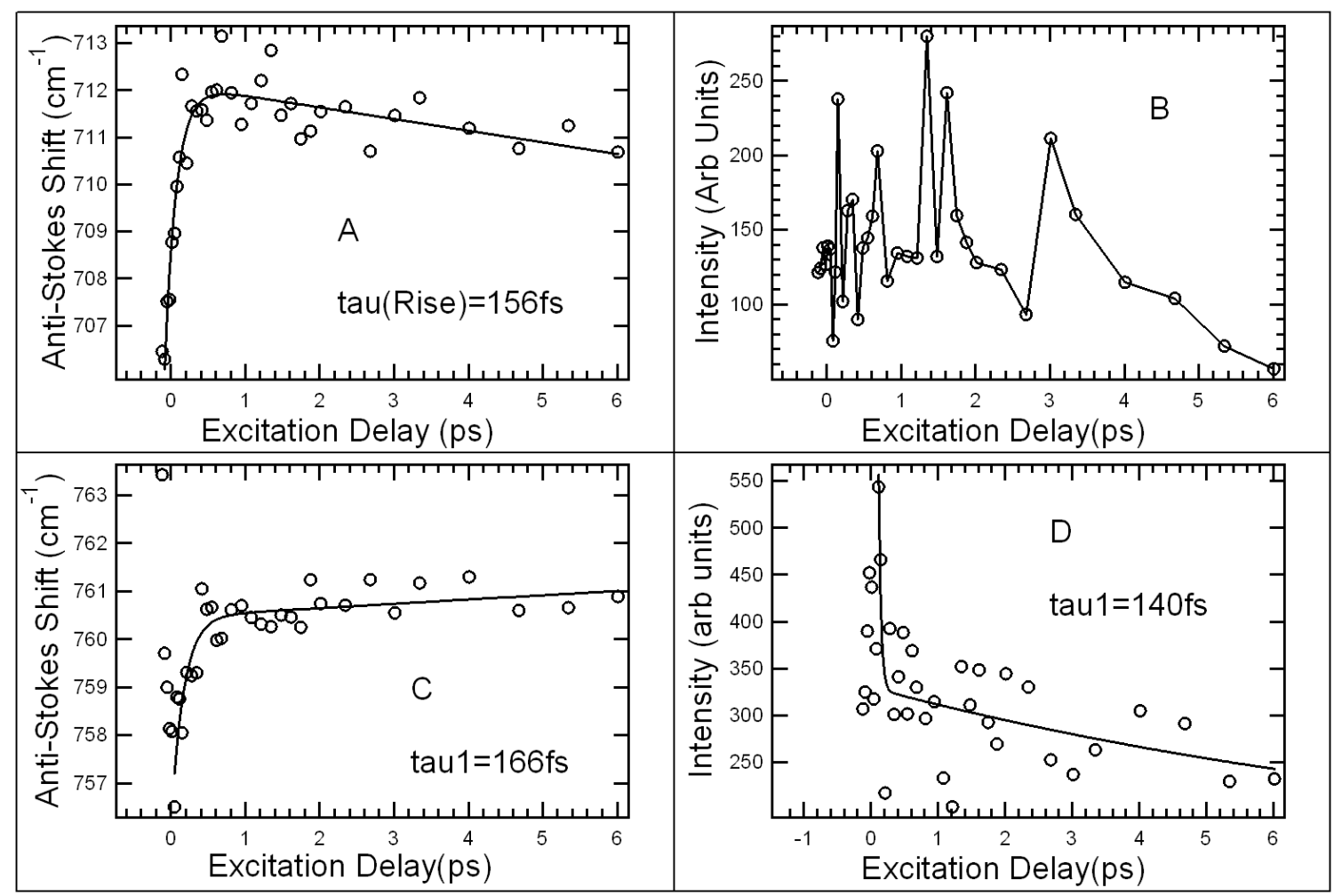

Figure 4.8 - Evolution of A) \& B) $710 \mathrm{~cm}^{-1}$ peak and of C) \& D) $760 \mathrm{~cm}^{-1}$ peak. A) The frequency of the 710 peak increases quickly (156fs) before falling off until the peak altogether disappears. B) The intensity of the 710 feature. A trace has been included to guide the eyes. The peak has a low intensity and fits were noisy. The peak increases in intensity for the first two picoseconds before decaying to zero at six picoseconds. C) The frequency of the 760 feature also shows a 166fs rise followed by a gradual increase in frequency for the duration of the experiment. D) The intensity of this mode begins very strong and then decreases quickly to reach a fairly stable value near 250 (arb units). 


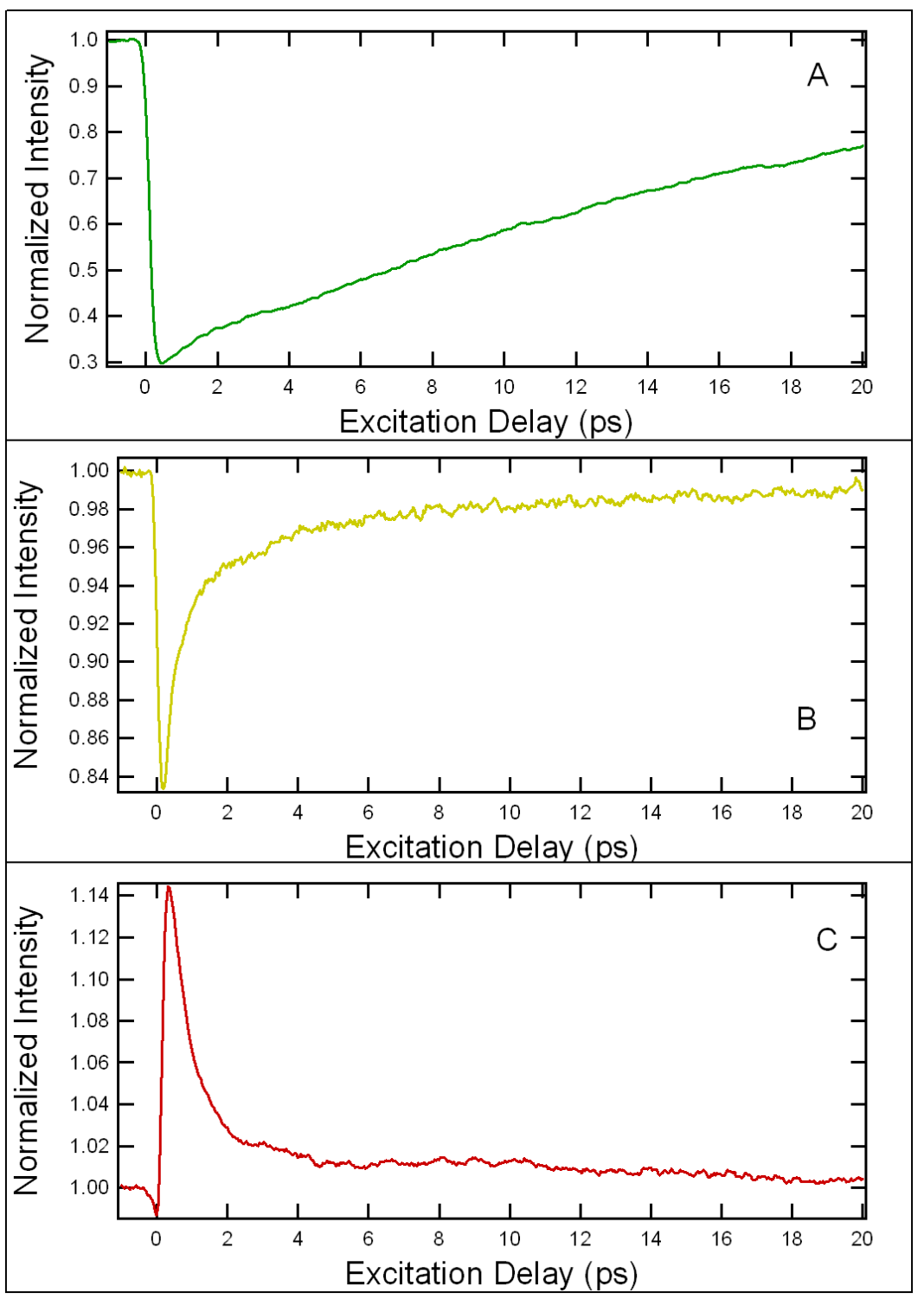

Figure 4.9 Transient spectra of DANS in ACN with 400nm pump and various probe wavelengths. Values greater than one indicate excited state emission while values less than one indicate excited state absorption. A) $400 \mathrm{~nm}$ pump, $511 \mathrm{~nm}$ probe B) $400 \mathrm{~nm}$ pump, $550 \mathrm{~nm}$ probe, C) $400 \mathrm{~nm}$ pump, $800 \mathrm{~nm}$ probe.

\begin{tabular}{|c|c|c|c|}
\hline & Green/Blue & Yellow/Blue & Red/Blue \\
\hline$\tau_{1}(\mathrm{ps})$ & 18 & .391 & .681 \\
\hline$\tau_{2}(\mathrm{ps})$ & N/A & 4 & 12 \\
\hline
\end{tabular}

Table 4.2 Fit lifetimes of transient traces shown in Figure 4.9. 


\section{CHAPTER 5: THE BEST OF THE REST}

\subsection{General Overview}

At the very core of research is the goal to learn. At times it can be difficult to determine what novel things to study while at others it can be nearly impossible to get a promising project to work in the way originally intended. Sometimes a system that seems like the perfect candidate to study turns out to be completely uncooperative. In this case the project may be pushed aside in favor of others that are (or at least seem) more promising.

During the workings of a research group there come times when the direction of the group as a whole may change. It is fairly common for each graduate student to work on his or her own project and to work with others as a sort of technical assistant. If one project begins to go well and the proverbial pieces start to fall into place, the focus of the group may shift away from struggling projects in favor of things similar to the successful one. This is the life of the research group.

Another interesting situation occurs when a system displays a behavior that was not expected. Sometimes a project creates questions that cannot be answered initially. Alternatively, the questions that arise may require additional studies that cannot be completed immediately. There may be times when a molecule under study shows such unexpected behavior that the group studying it may not have to best tools to study it. This is where collaboration comes in and can be an extremely useful tool for obtaining the best description of a particular system. 
This chapter is about learning from research. Each example described above represents one or more project that I have been involved with. It can be frustrating when projects do not work out the way they are intended, but even these can be great learning experiences. In the sections that follow several projects that represent 'ongoing work' will be described. These projects were either scrapped because they did not produce results for one reason or another, the overall direction of research in the group changed, or there has not yet been enough time to sufficiently study the system.

\subsection{Two Photon Absorption of Coumarin 120}

During the course of investigating the vibrational spectrum of Coumarin 120 (C120) an unexpected aspect of the system was observed: fluorescence. It is not extraordinary to observe fluorescence during the course of fs/ps-CARS experiments from many different types of samples, especially when dealing with highly conjugated organic chromophores. Fluorescence can often be observed with the naked eye from various samples following electronic excitation with $\omega_{\text {ex }}$, generally $400 \mathrm{~nm}\left(25000 \mathrm{~cm}^{-1}\right)$. This fluorescence is usually seen as a colored dot in the solution. For example, a solution of a specific chromophore dissolved in methanol may be bright yellow but the spot where it interacts with the $400 \mathrm{~nm}$ beam may appear to be white by the human eye.

The difference between the fluorescence normally observed following 400nm excitation and that observed in $\mathrm{C} 120$ is the electronic absorption process that is occurring. Typically, fluorescence follows absorption of a single photon of electromagnetic radiation. The molecule then relaxes via nonradiative processes until reaching the low 
energy vibrational states of the excited state before emitting. The emitted photon has lower energy (longer wavelength) than the incident photon.

$\mathrm{C} 120$, in polar solvents, can undergo one photon induced fluorescent processes, as shown in Figure 5.1. The absorption and emission spectra for $\mathrm{C} 120$ in $\mathrm{ACN}$ are shown in Figure 5.2. It is clearly seen that there is a small but tangible absorption cross-section at $400 \mathrm{~nm}$ and our excitation pulse will induce a one photon absorption followed by fluorescence. Any finite absorption intensity is gone by $450 \mathrm{~nm}$ for $\mathrm{C} 120$ in ACN.

The fluorescent process observed for $\mathrm{C} 120$ in $\mathrm{ACN}$ was first observed with the $510 \mathrm{~nm}$ beam. A transparent solution of the solute was placed in a rotating cell and, upon irradiation with the green $510 \mathrm{~nm}$ beam, a blue aura was clearly observed. Upon further investigation it was determined that a similar observation could be made with the $550 \mathrm{~nm}$ or $800 \mathrm{~nm}$ pulse present individually, indicating the possible presence of a multiphoton excitation process.

The conclusion that a two photon absorption and ensuing emission was occurring is justified when the absorption spectrum in this solvent is considered. There is no way for a single photon at $510 \mathrm{~nm}, 550 \mathrm{~nm}$ or $800 \mathrm{~nm}$ to access an electronically excited state, the absorption cross section at these wavelengths is much too low. If a two photon process is occurring, first consider the energy being deposited by two photons of the respective beams: $39200 \mathrm{~cm}^{-1}$ which is $255 \mathrm{~nm}$, corresponding to two $510 \mathrm{~nm}$ photons, $36362 \mathrm{~cm}^{-1}$ which is $275 \mathrm{~nm}$, two photons of $550 \mathrm{~nm}$ radiation, and finally, $25000 \mathrm{~cm}^{-1}$ resulting from two $800 \mathrm{~nm}$ photons, equivalent to a single $400 \mathrm{~nm}$ photon.

While it has been reported previously that C120 undergoes two photon absorption (TPA), most studies are concerned with using this molecule in biological detection 
schemes. ${ }^{1-3}$ The idea behind using this process in detection schemes if quite elegant. ${ }^{4}$ Many biological systems readily absorb ultraviolet radiation to access their excited states. By using two photon processes many types of chromophores may be discriminated against. This makes systems with high TPA cross-sections good candidates for single molecule detection studies. ${ }^{1-4}$

Emission spectra of C120 in ACN have been obtained using the 400nm and 510nm beams available from the fs/ps-CARS setup. ${ }^{5}$ Spectra were obtained using a $1 \mathrm{mM}$ solution of C120 (Exciton) dissolved in reagent grade acetonitrile (Fisher). The solution was placed in a $1 \mathrm{~cm}$ quartz cuvet and a fiber optic cable was placed $90^{\circ}$ relative to the incoming radiation to help discriminate against the pump beam. Collected fluorescence was spectrally dispersed and collected using an Ocean Optics USB-2000 spectrometer.

Results of fluorescence studies as measured by the Ocean Optics spectrometer are shown in Figure 5.3. Figure 5.3A is the fluorescence measured following two photon excitation with the $510 \mathrm{~nm}$ excitation pulse. There is a small amount of the incident beam that gets through to the detector. Figure 5.3B shows the normal type fluorescence due to one photon absorption of the $400 \mathrm{~nm}$ excitation pulse. The one photon absorption and two photon absorption appear to be very similar in shape. To our knowledge the two photon absorption of $\mathrm{C} 120$ at $510 \mathrm{~nm}$ has not been reported.

As discussed previously, one photon absorption from a $400 \mathrm{~nm}$ excitation pulse accesses the very red edge of the absorption spectrum in C120. This indicates that the excited species will be at or near its lowest vibrational levels. Monitoring the vibrational evolution following this excitation would allow the assumption to be made that there are no nonradiative processes occurring including no vibrational energy redistribution in 
order to reach the lowest vibrational levels. Two photon excitation with the $510 \mathrm{~nm}$ pulse, on the other hand, is equivalent to a single $255 \mathrm{~nm}$ photon. In this case the molecule is excited very high into the $S_{1}$ and may be in the very low $S_{2}$ levels. In either case it would be possible to measure the relaxation of the molecule immediately following excitation to reach the lowest vibrational levels within the first excited singlet state. Dynamics of C120 following one photon excitation to the lowest levels in the first excited singlet state could then be compared with the dynamics following TPA to high lying levels of this state.

Comparing the dynamics of excitation with $400 \mathrm{~nm}$ versus $510 \mathrm{~nm}$ would be very interesting. Additionally, a $267 \mathrm{~nm}$ excitation pulse could be prepared to compare one photon excitation to high lying states against the nearly equivalent two photon process. Drawbacks with producing the $267 \mathrm{~nm}$ pulse include low conversion efficiency (there would not be much power of this pulse for excitation) and the optics needed. At this wavelength the beams cannot be seen by the naked eye and experiments can be dangerous. If the equivalent process could be studied using a $510 \mathrm{~nm}$ visible pulse, it would be beneficial.

It was not expected that this project would lead down this particular path. If infinite time to perform experiments were available, the excited state dynamics would be monitored for this system in several different solvents using the one photon and two photon excitation schemes. These studies could, theoretically, help to determine the relaxation processes C120 undergoes in its excited state before fluorescence occurs. These are studies that could still be conducted. 


\subsection{Effects of Nanoconfinement on Twisting Dynamics}

Inclusion complex formation has been the subject of numerous studies in past years. ${ }^{6-12}$ One of the most common host compounds are cyclodextrins which are cyclic oligosaccharides composed of six or more glucopyranose units bonded through $\alpha-1,4-$ glycosidic bonds. ${ }^{8}$ The most common cyclodextrins are alpha $(\alpha-\mathrm{CD}$, six glucopyranose units), beta ( $\beta$-CD, seven glucopyranose units), and gamma ( $\gamma$-CD, eight glucopyranose units). Inclusion can confine vibrations and torsions in molecules as well as increase solubility. ${ }^{8}$ Cyclodextrins are also capable of protecting guest molecules from environmental effects. ${ }^{7}$ Of interest is the interaction different functional groups undergo with the cyclodextrin, specifically, monitoring how these interactions change upon perturbation of the system through electronic excitation. ${ }^{6-12}$ Pump-probe experiments allow these interactions to be studied. Figure 5.4 outlines the structure typical of cyclodextrins.

The premise behind this study was to take a chromophore which had been studied within our group and determine the effects of inclusion on its excited state dynamics. The processes undergone in the excited state can be greatly affected by the type of complex formed. Typical inclusion complexes include but are not limited to 2:1, 1:2 and 2:2 host:guest ratios. ${ }^{6}$ Any twisting, vibrating or motion in general of the guest should be hindered within the host cyclodextrin. Molecules singled out for study included pnitroaniline (PNA) and N,N-dimethyl-p-nitroaniline (DMPNA), systems that have been studied extensively in our group. ${ }^{5}$ The structure of these species is shown in Figure 5.5.

There are two solvents in which cyclodextrin inclusion complexes can be effectively formed: water and dimethyl sulfoxide. The approach to forming the complex 
is as simple as mixing the guest and host molecules in the solvent of choice. In many cases a solvent in which the host is soluble but the guest is fairly insoluble is chosen. In this way it can be assumed that any property measured is due to included species. Inclusion can be determined experimentally by measuring the absorption spectrum of the solvated guest and gradually adding cyclodextrin. The normal behavior is a gradual red shift of the absorption spectrum as the complex is formed.

Initial studies were aimed at determining if inclusion was actually occurring in the system. For this steady state absorption experiments were conducted. For both PNA and DMPNA in water the presence of the complex was confirmed. Figure 5.6 shows that for PNA there is a definite change of the absorption spectrum in the presence of cyclodextrin. This is evidenced by a red shift of the absorption spectrum relative to the free species in solution. DMPNA in water was also confirmed to undergo inclusion complex formation, as is shown in Figure 5.7. The presence of the inclusion complex for DMPNA is again confirmed by the red shift of its absorption maximum.

Problems were encountered in this project related to the experimental parameters involved, specifically discerning the included species from free species. PNA is essentially infinitely soluble in water and it is likely the species forming the host:guest complex is in equilibrium with the free species. DMPNA, on the other hand, is minimally soluble in water, less than $50 \mu \mathrm{M}$ in fact. In this case the complex would stabilize the guest molecule and help it become solvated.

Further studies were conducted to determine if an effect of inclusion could be noticed using time resolved methods. Figure 5.8 shows the two color transient emission spectra for PNA with various proportions of $\beta$-CD. Figure 5.8A shows the $400 \mathrm{~nm}$ pump, 
$510 \mathrm{~nm}$ probe spectra while Figure 5.8B shows the $400 \mathrm{~nm}$ pump, $550 \mathrm{~nm}$ probe spectra. The only tangible difference in these spectra is the maximum intensity of the emission measured. It is likely that this intensity difference is not related to the inclusion process at all and that as molecules enter the host cavity they no longer undergo the excitation process.

Figure 5.9 illustrates the effect of inclusion of DMPNA on the dynamics of its stimulated emission spectra. Positive is again emission. Figure 5.9A is $400 \mathrm{~nm}$ pump, 510 $\mathrm{nm}$ probe while 5.9B is $400 \mathrm{~nm}$ pump, $550 \mathrm{~nm}$ probe. The same observation about the complex formed with PNA can be said about this species. The intensity of the emission peak changes based upon the number of inclusion complexes that have formed while the dynamics do not change.

It was determined that both PNA and DMPNA undergo an inclusion process with cyclodextrin present as shown in Figures 5.6 and 5.7. The absorption spectrum of each species exhibits a red shift upon inclusion, indicating a definite interaction with the cavity of the host molecule. Two color pump probe measurements on both PNA and DMPNA have indicated that the dynamics associated with the relaxation of the excited state with cyclodextrin present is not affected by inclusion. This indicates that several things may be occurring. Firstly, an equilibrium process in which the guest moves in and out of the host molecule is happening and the free species is being probed. Another possibility is that there are free guest molecules in solution that have not undergone inclusion and these are being probed. Still another possibility is that the guests are not being sufficiently hindered within the cavity and are essentially free molecules sitting within a cyclodextrin. 


\subsection{Dye Sensitized Nanoparticles}

The development of nanotechnology has been accompanied by a desire to understand how that technology works. ${ }^{13-19}$ Some of the most easily produced alternative energy sources are dye-sensitized solar cells. ${ }^{15}$ The classic "Gratzel Cell" has been used in classrooms from elementary school to university to demonstrate the practicality of generating electricity from the sun's radiation. ${ }^{18}$ These cells are simple to make, easy to use, and the components are widely available. A significant amount of effort has been invested in understanding the initial process solar cells: electron injection. In the first step of a solar cell's operation a dye injects an electron into the conduction band of a nanoparticle.

If an electron is excited out of the valence band into the conduction band of a nanoparticle an electron-hole pair is formed. ${ }^{12-15}$ Eventually the electron will make its way back to fill the hole via different methods. If the system is connected to a solar cell the electron can be separated from the hole in such a way as to perform work. Unfortunately most nanoparticles only absorb ultraviolet radiation and are not very efficient for using in solar cells. If a dye that exhibits absorption in the visible range, however, is attached to the nanoparticle, the efficiency of the system can be increased.

Generally titanium dioxide serves as the nanoparticle semiconductor. ${ }^{18}$ This material is easy to produce, widely available, inexpensive, and behaves very well in solar cells. Selection of dyes varies and often includes a form of a ruthenium dye, coumarin, or anthraquinone. When the complex is formed between the nanoparticle and the dye, it needs to be stable. Dyes that can form five, six, or seven membered rings upon complexation are generally the best candidates for producing a solar cell. ${ }^{14}$ 
We set out to determine how the vibrational spectrum of the dye is affected upon attaching it to a nanoparticle. The ultimate goal was to monitor the excited state vibrational spectrum of the dye following injection of an electron into the conduction band of a $\mathrm{TiO}_{2}$ nanoparticle. Throughout the course of the investigation, the obstacles encountered were highly varied. Initially the preparation of $\mathrm{TiO}_{2}$ acted as a limiting reagent but an efficient preparation procedure was quickly developed. Exploratory efforts to determine the best dye to attach to the nanoparticles were largely unsuccessful. Obtaining fs/ps-CARS spectra of dyes attached to nanoparticles was in itself very difficult. Finding signal from an ideal chromophore solvated in a polar organic solvent, such as $\mathrm{C} 153$ in $\mathrm{MeOH}$, can be a difficult proposition at times. Replacing the highly resonant C153 with the nonresonant C343, catechol (o-dihydroxybenzene), and lastly alizarin (1,2-dihydroxy anthraquinone) resulted in several efforts.

The problem was not in acquiring the time resolved electronic spectra of these species, which had been done previously. The goal of this study was essentially to find the best nanoparticle, dye, and solvent to measure the vibrational spectrum of the dye before and after electron injection. All efforts to look at catechol and C343 resulted in no successful acquisition of a vibrational spectrum. The study took an interesting turn when a commercially available zirconium dioxide (zirconia) dispersion was purchased.

The band gap of zirconia is much higher than titania, as shown in Figure 5.10. It is not possible for any of the three dyes mentioned to inject an electron into the conduction band of the $\mathrm{Zr}$ nanoparticle. ${ }^{14-16}$ It is still possible, however, to measure the vibrational spectrum of a dye that is attached to zirconia. Alizarin (Figure 5.11) was selected as the sensitizing dye. 
Preparation of the alizarin-sensitized zirconia was done by dissolving solid alizarin to a concentration of $2 \mathrm{mM}$ in the commercially obtained zirconia dispersion. Simply stirring the mixture resulted in a change in color from the clear dispersion to a deep purple sensitized species. The sample was placed in a rotating cell and fs/ps-CARS spectra were obtained using the methods described in the previous chapters.

The ground state fs/ps-CARS spectrum of alizarin on zirconia is shown in Figure 5.12. To our knowledge this is the only reported vibrational spectrum of this dye on this specific nanoparticle. Most of the observed peaks are ring vibrations of the complex. Notable exceptions include the peaks at 1291 and $1323 \mathrm{~cm}^{-1}$, which are assigned as combination ring and $\mathrm{C}-\mathrm{O}$ stretch. Assignments are shown in Table 5.1.

The vibrations observed, when considered in conjunction with two previous studies on alizarin attached to $\mathrm{TiO}_{2}$ and silver nanoparticles, seem to be a hybrid between the two. ${ }^{15,16}$ This indicates that there are likely multiple types of complexes being formed, as shown by Sanchez-Cortes et al. There is also the distinct possibility that the signal is coming from free alizarin, as in the cyclodextrin studies. This study shows that the fs/psCARS technique is sensitive enough to measure the vibrations of molecules attached to nanoparticles.

Unfortunately all attempts to measure the excited state vibrational structure of dyes attached to nanoparticles were futile. Reasons for this are include the dye being nonresonant with the probe technique and the molecule simply undergoing nothing interesting in its excited state on sub picosecond timescales. Subsequent studies were not conducted as the direction of the group turned to other systems, including Michler's 
Ketone and 4-dimethylamino-4'-nitrostilbene, as discussed earlier in this thesis.

Sometimes when experiments do not work for the best a new direction should be tried.

\subsection{References}

1. A. Fischer, C. Cremer and H. K. Stelzer, Appl. Opt., 34, 1989 (1995)

2. K. A. Nguyen, P. N. Day and R. Pachter, J. Chem. Phys., 126, 094303 (2007)

3. L. Brand, C. Eggeling, C. Zander, K. H. Drexhage and C. A. M. Seidel, J. Phys. Chem. A, 101, 4314 (1997)

4. P. R. Monson and W. M. McClain, J. Chem. Phys, 53, 29 (1970)

5. B. D. Prince, A. Chakraborty, B. M. Prince, and H. U. Stauffer, J. Chem. Phys, 125, 044502 (2006)

6. A. Douhal, Ed, Cyclodextrin Materials Photochemistry, Photophysics, and Photobiology, Elsevier: 2006.

7. A. Nitzan and M. A. Ratner, Science, 300, 1384 (2003)

8. J. Szejtli, Chem. Rev., 98, 1743 (1998)

9. P. Lo Meo, F. D’Anna, S. Riena, M. Gruttadauria, and R. Noto, Tetrahedron, 58, 6039 (2002)

10. M. L. Bender and M. Komiyama, Cyclodextrin Chemistry, Springer-Verlag, 1978

11. Z. R. Grabowski, K. Rotkiewicz and W. Rettig, Chem. Rev., 103, 3899 (2003)

12. A. Nag and K. Battacharyya, J. Chem. Soc. Faraday Trans., 86, 53 (1990)

13. H. N. Ghosh, J. B. Asbury and T. Lian, J. Phys. Chem. B., 102, 6482 (1998)

14. D. Pant and N. E. Levinger, Chem. Phys. Lett, 292, 200 (1998)

15. L. C. T. Schoute and G. R. Loppnow, J. Chem. Phys., 117, 842 (2002)

16. M. V. Canamares, J. V. Garcia-Ramos, C. Domingo and S. Sanchez-Cortes, J. Raman Spectrosc., 35, 921 (2004) 
17. H. N. Ghosh, J. Phys. Chem. B., 103, 10382 (1999)

18. G. P. Smestad and M. Grätzel, J. Chem. Ed., 75, 752 (1998)

19. J. M. Rehm, G. L. McLendon, Y. Nagasawa, K. Yoshihara, J. Moser and M. Grätzel, J. Phys. Chem., 100, 9577 (1996) 


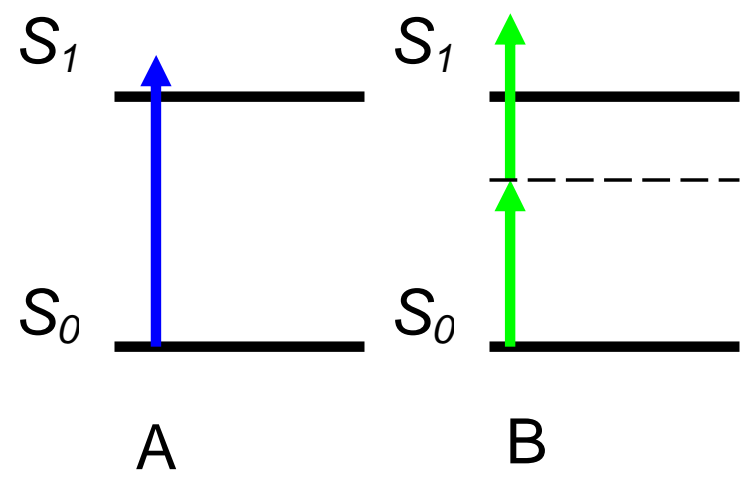

Figure 5.1 - Typical electronic excitation processes A) One photon absorption B) Two photon absorption.

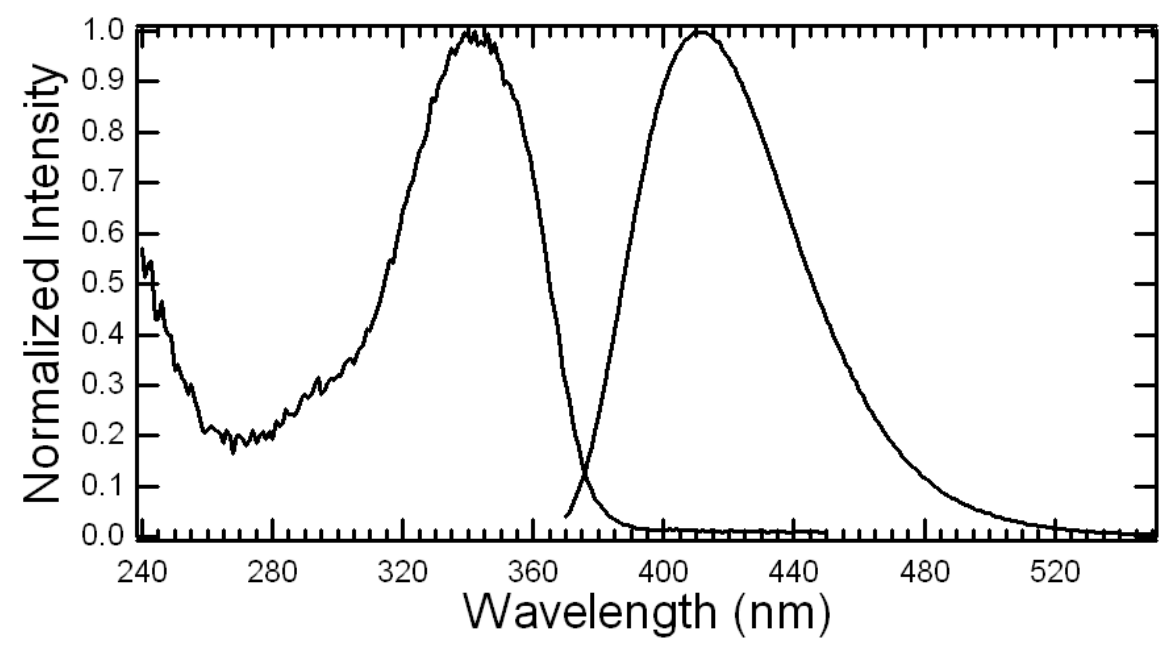

Figure 5.2 - Absorption and emission spectra of C120 in ACN. 


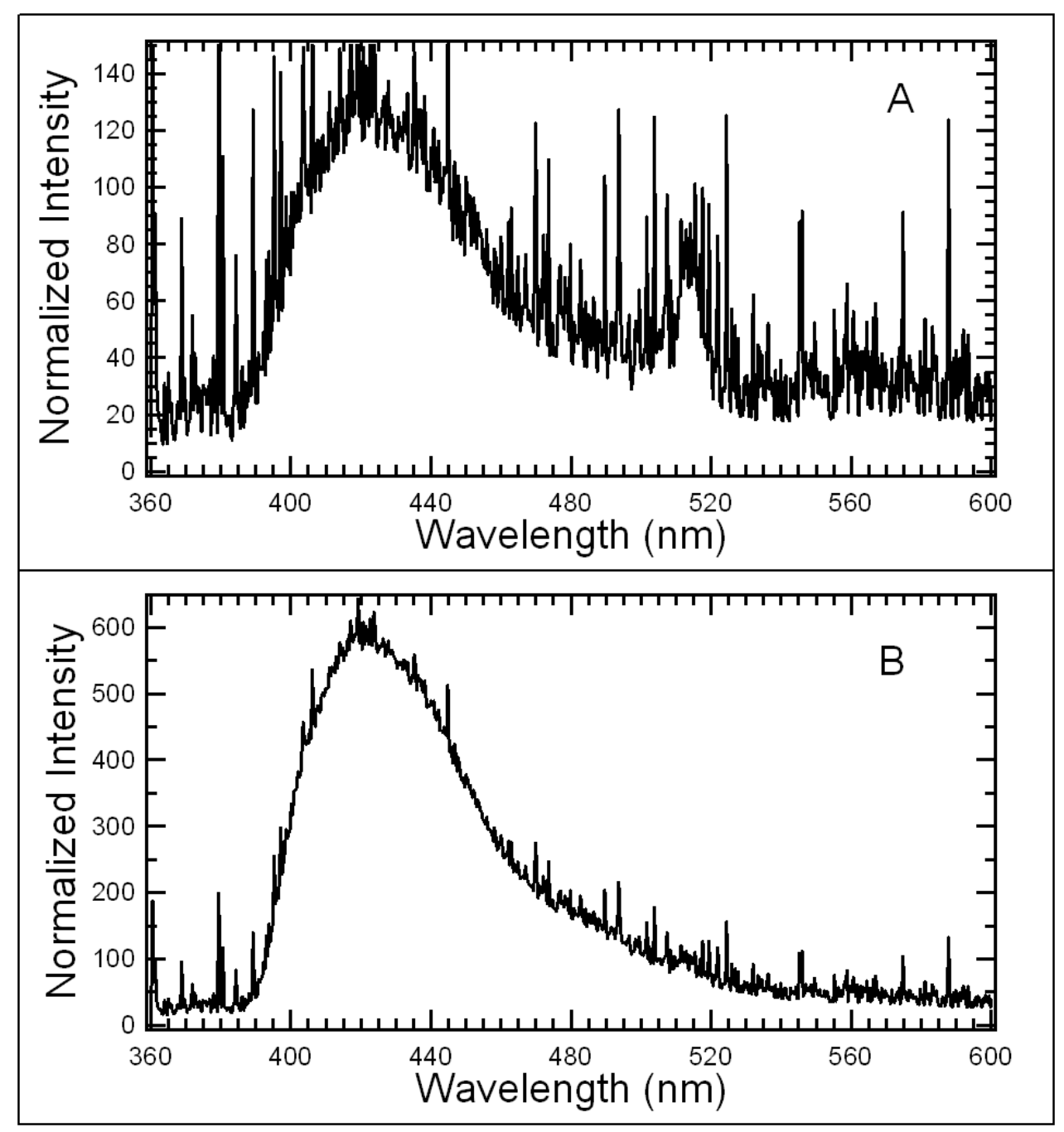

Figure 5.3 - Emission spectra measured with A) $510 \mathrm{~nm}$ excitation B) $400 \mathrm{~nm}$ excitation. 

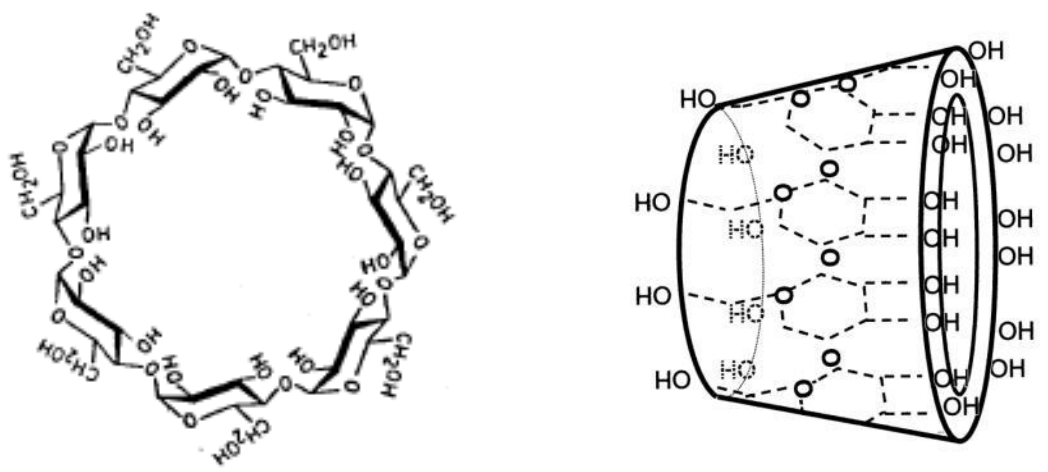

Figure 5.4 - Different views of cyclodextrin structure. Beta Cyclodextrin structure (left) and overview of cyclodextrin geometric structure using Gamma Cyclodextrin (right,). The 2-OH and 3-OH groups line one end, forming the wide cavity while the 6- $\mathrm{CH}_{2} \mathrm{OH}$ groups line the opposite end, forming the narrow cavity. External Hydroxyl groups make the cyclodextrin soluble in water. The inner cavity is lined with 3-H and 4$\mathrm{H}$ groups as well as intersaccharide oxygens, causing the interior to be hydrophobic.

A) 0<smiles>Nc1ccc([N+](=O)[O-])cc1</smiles>

B)<smiles>CN(C)c1ccc([N+](=O)[O-])cc1</smiles>

Figure 5.5 - Structure of A) PNA and B) DMPNA 


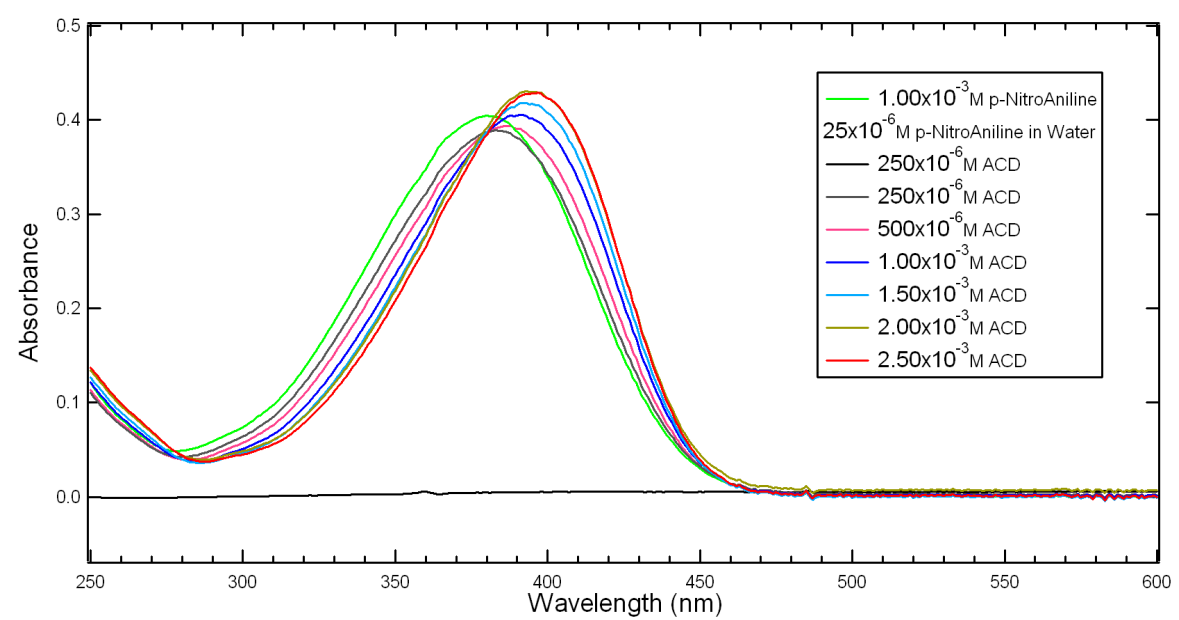

Figure 5.6 - Concentration dependence of absorption spectrum of PNA in various concentrations of $\alpha$-CD. As the concentration of cyclodextrin is increased, the effect of inclusion is evidenced by the red shift of the absorption spectrum.

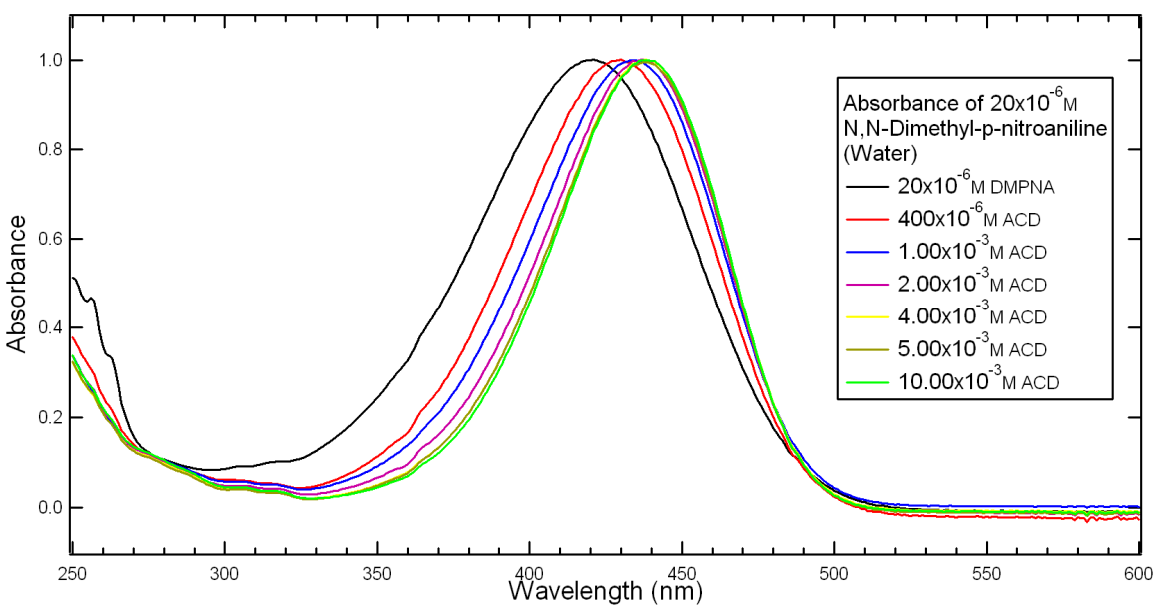

Figure 5.7 - Concentration dependence of absorption spectrum of DMPNA in water. It is seen that the absorption maximum shifts to the red as inclusion occurs. 


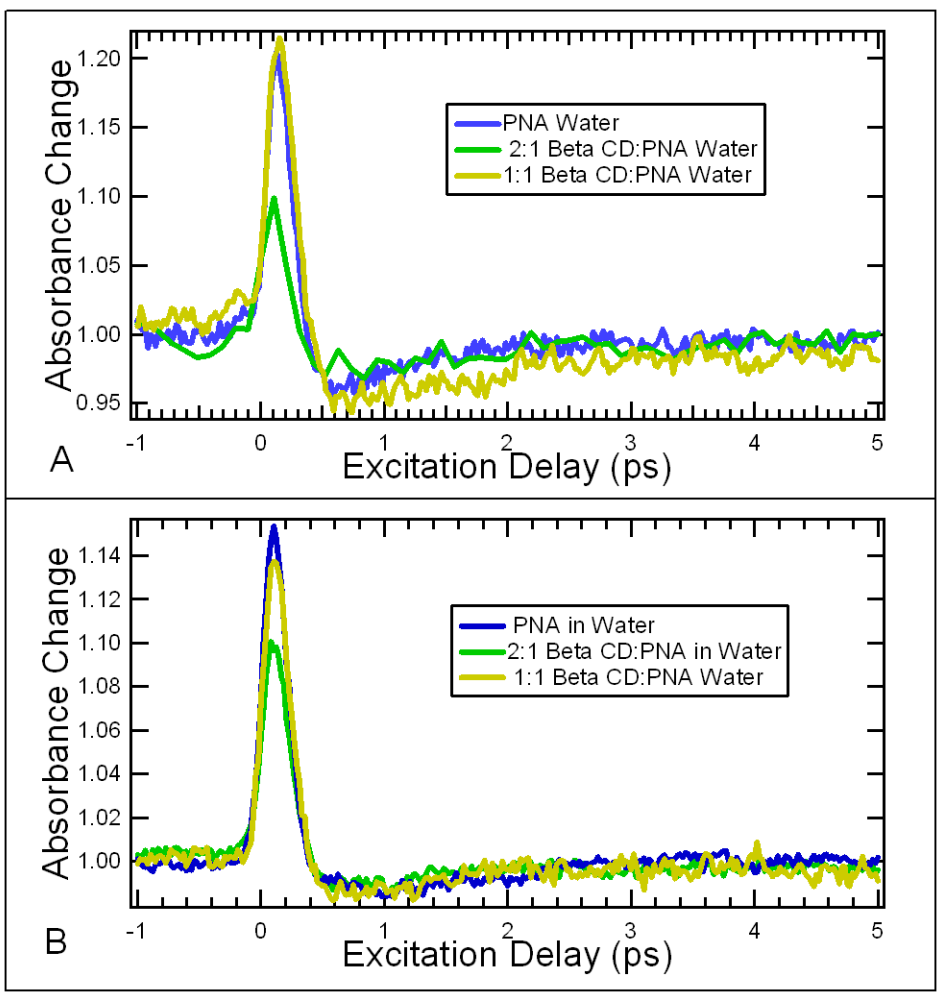

Figure 5.8 - Transient emission spectra of PNA in water with various proportions of $\beta$-CD. Greater than 1.00 is emissive, less than 1.00 is absorptive. A) $400 \mathrm{~nm}$ pump, $510 \mathrm{~nm}$ probe. B) $400 \mathrm{~nm}$ pump, $550 \mathrm{~nm}$ probe. It is clear that the dynamics are not changing dramatically but that the intensity changes. This effect is likely dependent upon PNA concentration as more of the guest becomes included, the number of molecules undergoing excitation is reduced. As a result the peak absorbance change is reduced since the signal is proportional to concentration. 

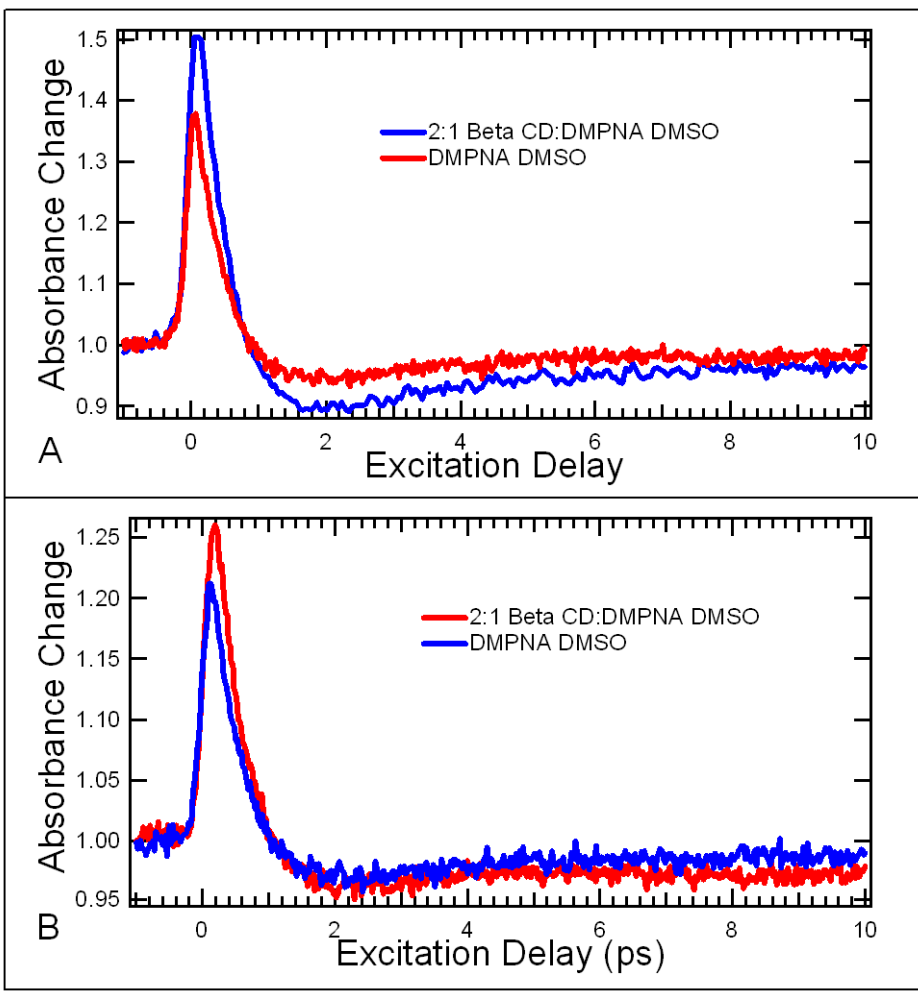

Figure 5.9 - Transient emission spectra of DMPNA in DMSO with various proportions of $\beta$-CD. Greater than 1.00 is emissive, less than 1.00 is absorptive. A) $400 \mathrm{~nm}$ pump, $510 \mathrm{~nm}$ probe. B) $400 \mathrm{~nm}$ pump, 550 $\mathrm{nm}$ probe. It is clear that the dynamics are not changing dramatically but that the intensity changes. As with PNA in water, the intensity effect is likely related to the inclusion process. 


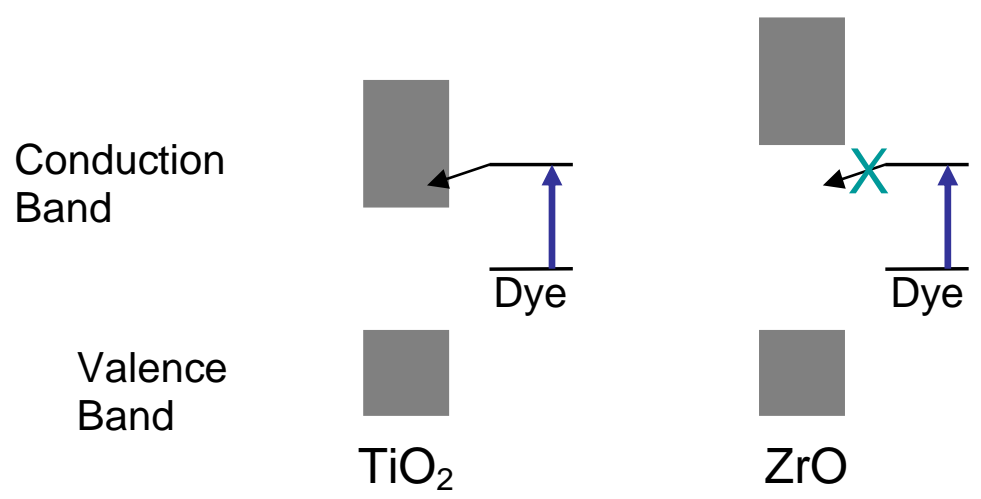

Figure 5.10 - Schematic of the electron injection process in $\mathrm{TiO}_{2}$ and $\mathrm{ZrO}_{2}$. The dye, in this case Alizarin, cannot inject an electron into the valence band of the zirconium particle because the conduction band is too high in energy.<smiles>O=C1c2ccccc2C(=O)c2c1ccc(O)c2O</smiles>

Figure 5.11 - Structure of alizarin

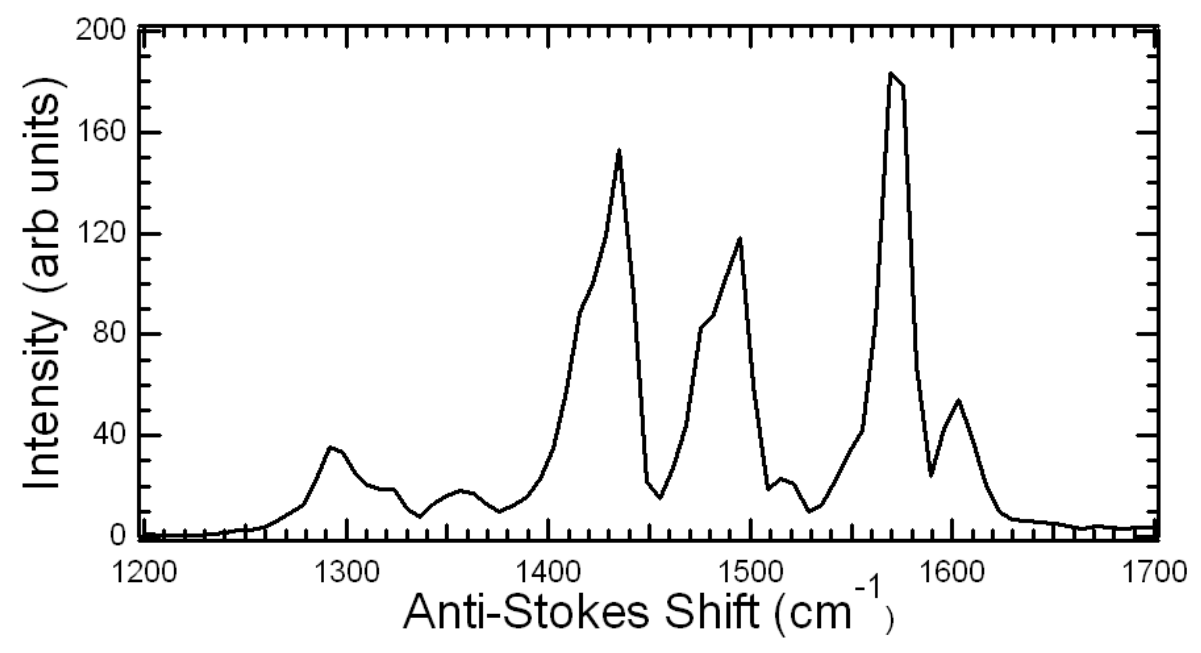

Figure 5.12 - fs/ps-CARS spectrum of Alizarin attached to zirconia in aqueous solution. 


\begin{tabular}{|c|c|}
\hline Observed Frequency $\left(\mathbf{c m}^{-1}\right)$ & Assignment \\
\hline 1602 & Ring Stretch \\
\hline 1569 & Ring Stretch \\
\hline 1515 & Ring Stretch \\
\hline 1495 & Ring Stretch \\
\hline 1475 & Ring Stretch \\
\hline 1435 & Ring Stretch \\
\hline 1415 & Ring Stretch \\
\hline 1323 & Ring + C-O \\
\hline 1291 & Ring + C-O \\
\hline
\end{tabular}

Table 5.1 - Vibrational assignments for alizarin attached to $\mathrm{ZrO}_{2}$ 


\section{CHAPTER 6: CONCLUSIONS}

Chapter 2 details the study of the vibrational evolution of the excited state of Michler's Ketone with $400 \mathrm{~nm}$ excitation and subsequent fs/ps-CARS probe. Initial studies using two color pump-probe methods to monitor the electronic evolution of this molecule in the $S_{1}$ indicated the presence of several excited state configurations in two polar solvents: $d_{4}$-MeOH and $d_{6}$-DMSO. Ground state fs/ps-CARS spectra were obtained in both solvents and vibrations measured were assigned with the assistance of DFT calculations. The pump-fs/ps-CARS experiments indicated slightly different, but related behavior of Michler's Ketone in these two solvents. In $d_{4}-\mathrm{MeOH}$ there is a rapid change of the $\mathrm{Ph}-\mathrm{N}$ stretch at $1380 \mathrm{~cm}^{-1}$ concomitant with a slightly slower evolution of the $\mathrm{Ph}$ $\mathrm{CC}+\mathrm{Ph}-\mathrm{N}$ combination mode at $1513 \mathrm{~cm}^{-1}$. The rapid evolution of the pure $\mathrm{Ph}-\mathrm{N}$ stretch is attributed to the planarization of the molecule to produce the intramolecular charge transfer state while the actions of the $\mathrm{Ph}-\mathrm{N}+\mathrm{Ph}-\mathrm{CC}$ mode are indicative of the production of the ICT state following planarization. After 3 ps the vibrations are no longer observed, indicating we are not measuring the TICT state in this molecule. In the aprotic $d_{6}$-DMSO, on the other hand, we clearly measure the $\mathrm{Ph}-\mathrm{N}+\mathrm{Ph}-\mathrm{CC}$ mode evolving from the ICT state to the TICT state. This mode remains for over 300 ps. The

excited state low frequency mode at $285 \mathrm{~cm}^{-1}$ indicates that the molecule reaches a stable configuration around $50 \mathrm{ps}$ in $d_{6}$-DMSO and remains in this state for over $300 \mathrm{ps}$. These studies clearly confirm that this molecule evolves from the initially excited configuration to the ICT state and eventually to the TICT state. 
Chapter 3 outlines the study of the vibrational structure of coumarin 120 . The optimized DFT B3LYP structure using the triple zeta 6-311G(d,p) basis set is compared with the experimentally determined (XRD) structure and the two are found to match very well. For the first time, FTIR and spontaneous Raman spectra are reported and the measured vibrations are assigned using quantum chemical DFT calculations. Further calculations using the polarizable continuum model are used to predict how the vibrational structure changes in different solvents. It was found that the carbonyl frequency decreases as solvent polarity increases as a result of the lengthening of the $\mathrm{C}=\mathrm{O}$ bond length. The vibration that best resembles the pure $\mathrm{Ph}-\mathrm{N}$ stretch increases in frequency as solvent polarity is increased because the $\mathrm{Ph}-\mathrm{N}$ bond length decreases due to charge transfer.

The complicated low frequency excited state dynamics of 4-dimethyl-4'nitrostilbene in acetonitrile are discussed in Chapter 4. Ground state vibrations change significantly upon excitation, indicating a significant change of the structure of the molecule. Three pure excited state modes are observed and tentatively assigned. It is deduced that the excited state structure is greatly different from the ground state geometry. The initially accessed Franck-Condon state is not measured, however, and it is assumed that the molecule evolves out of this state within the duration of the pulse. Several projects that were cut short for one reason or another are discussed in Chapter 5. The two photon absorption of $\mathrm{C} 120$ is detailed and it is shown that this molecule undergoes efficient two photon excitation with $510 \mathrm{~nm}$ radiation. It is suggested that it may be interesting to compare the dynamics following one photon excitation at $400 \mathrm{~nm}$ and two photon excitation at $510 \mathrm{~nm}$. 
The effects of inclusion within cyclodextrin cavities are also outlined in Chapter 5. It was determined that the two chromophores: PNA and DMPNA are not greatly affected inside a nanocavity. Finally, the dye Alizarin is attached to zirconia nanoparticles and its vibrational structure is measured. Measured vibrations are assigned in analogy to previous work using titania and silver nanoparticles. 


\section{ACKNOWLEDGEMENTS}

None of the experimental efforts described herein would have been remotely possible without the guidance of my major professor Hans Stauffer. It is quite impossible to express the amount of insight Hans had into the studies being conducted in the lab. Every time I would present data to him he would pose interesting questions that made me thirst for more knowledge about whatever system I was studying. Whenever I had a question about research, spectroscopy, presentations or even the current hot topic in sports Hans was always ready with great comments.

I would very much like to thank Professor Mark Gordon for all of the time and effort he has invested in me. It seems like an eternity since I sat in his computational chemistry class and learned exactly what theoretical chemistry was about. He sparked in me the desire to study systems using experimental and theoretical tools in order to learn as much about each molecule as possible. This two-pronged approach has become a mainstay in my research and I look forward to teaching it to undergraduates in the future. Discussions about research, life, and graduation are greatly appreciated. Whenever Mark would walk into the classroom for a lecture he would say that 'Alex is up to something' and I guess he was right.

Very special thanks are extended to Ben Prince. Ben is a great friend and a wonderful research associate and I feel lucky to have had the opportunity to work with him. He really seemed like a sponge of knowledge on every topic imaginable. Experimental aspects or interpreting data were commonplace discussions with him, but he always had insight during conversations about world history, music, and even sports. 
And his classic "Go Cubs" will forever reverberate in my mind as a timeless answer to even the most unanswerable problem.

I would not be where I am right now if not for the help of my committee: Joe Burnett, Mark Gordon, William Jenks, Victor Lin, Xueyu Song, and Hans Stauffer. Each member of my committee has helped guide my work in interesting directions and have helped answer burning questions. I'd like to thank Professor Jenks for acting as my Preparing Future Faculty mentor while I was entrenched in the job search. His advice went a long way to helping me prepare for interviews.

The most sincere gratitude is extended to the entire Gordon research group, whom I have interacted with on countless occasions. From group meetings to queries regarding everything from input files to levels of theory and anything else GAMESS related, they were always available and always willing to help.

Many of my research efforts were enriched by collaboration. Steady state electronic spectra obtained in the labs of Professor Jacob Petrich were helpful on numerous occasions. I would like to sincerely thank Ramkrishna Adhikary and Sayantan Bose for access to their absorbance and emission instruments and for useful discussions. The Coumarin 120 project was greatly assisted by FTIR and spontaneous Raman spectra which were acquired with the assistance of Justin Valenstein of the Lin Group and Kris McKee Smith Group, respectively. Thank you to everyone who has collaborated with us through the years.

I would like to recognize Joe Burnett for everything he has helped me with in my time at Iowa State. I have served as a teaching assistant for Joe on several occasions. He also gave me the opportunity to serve as a lecturer to further my experience in Preparing 
Future Faculty. As a lecturer I learned much more about teaching than I did in all the time I spent as a teaching assistant. Joe was also my faculty mentor for PFF in my final semester and discussions with him were supremely helpful.

I would like to thank all of the people I have served as a teaching assistant for. There have been a few: Joe Burnett, Steve Walstrum, Kingston Jesudoss, Christina Bonaccorsi, Stacey Stoffregren, and Hans Stauffer. Additionally I would like to thank Toni Smith, my co-instructor for Chem 155. All of these people helped me become the best instructor I can and I look forward to taking my experiences into a new challenge as a faculty member at Alverno College. Finally, I would like to extend a special thanks to the friends and family who have supported me through all of my endeavors. To Alyssa and Alana, thanks for keeping me driven and being supportive through everything. 